\title{
Heavy metals in intermediate He-rich hot subdwarfs: the chemical composition of HZ 44 and HD 127493*
}

\author{
M. Dorsch ${ }^{1}$, M. Latour ${ }^{2}$, and U. Heber ${ }^{1}$ \\ ${ }^{1}$ Dr. Remeis-Sternwarte \& ECAP, Astronomical Institute, University of Erlangen-Nürnberg, Sternwartstr. 7, \\ 96049 Bamberg, Germany \\ e-mail: matti.dorsch@fau.de \\ 2 Institut für Astrophysik, Georg-August-Universität, Friedrich-Hund-Platz 1, 37077 Göttingen, Germany
}

Received 17 April 2019 / Accepted 16 July 2019

\begin{abstract}
Context. Hot subluminous stars can be spectroscopically classified as subdwarf B (sdB) and O (sdO) stars. While the latter are predominantly hydrogen deficient, the former are mostly helium deficient. The atmospheres of most sdOs are almost devoid of hydrogen, whereas a small group of hot subdwarf stars of mixed $\mathrm{H} / \mathrm{He}$ composition exists, showing extreme metal abundance anomalies. Whether such intermediate helium-rich (iHe) subdwarf stars provide an evolutionary link between the dominant classes is an open question. Aims. The presence of strong $\mathrm{Ge}, \mathrm{Sn}$, and $\mathrm{Pb}$ lines in the UV spectrum of HZ 44 suggests a strong enrichment of heavy elements in this iHe-sdO star and calls for a detailed quantitative spectral analysis focusing on trans-iron elements.

Methods. Non-local thermodynamical equilibrium model atmospheres and synthetic spectra calculated with TLUSTY/SYNSPEC were combined with high-quality optical, ultraviolet (UV), and far-UV (FUV) spectra of HZ 44 and its hotter sibling HD 127493 to determine their atmospheric parameters and metal abundance patterns.

Results. By collecting atomic data from the literature we succeeded in determining the abundances of 29 metals in HZ 44, including the trans-iron elements $\mathrm{Ga}, \mathrm{Ge}, \mathrm{As}, \mathrm{Se}, \mathrm{Zr}, \mathrm{Sn}$, and $\mathrm{Pb}$ and providing upper limits for ten other metals. This makes it the best-described hot subdwarf in terms of chemical composition. For HD 127493 the abundance of 15 metals, including $\mathrm{Ga}, \mathrm{Ge}$, and $\mathrm{Pb}$ and upper limits for another 16 metals were derived. Heavy elements turn out to be overabundant by one to four orders of magnitude with respect to the $\mathrm{Sun} . \mathrm{Zr}$ and $\mathrm{Pb}$ are among the most enriched elements.

Conclusions. The $\mathrm{C}, \mathrm{N}$, and $\mathrm{O}$ abundance for both stars can be explained by the nucleosynthesis of hydrogen burning in the $\mathrm{CNO}$ cycle along with the stars' helium enrichment. On the other hand, the heavy-element anomalies are unlikely to be caused by nucleosynthesis. Instead diffusion processes are evoked, with radiative levitation overcoming gravitational settlement of the heavy elements.
\end{abstract}

Key words. stars: abundances - stars: atmospheres - stars: individual: HZ 44 - stars: individual: HD 127493 - stars: evolution subdwarfs

\section{Introduction}

Hot subdwarf stars of spectral type $\mathrm{O}$ and $\mathrm{B}$ ( $\mathrm{sdO}$ and $\mathrm{sdB}$ ) represent late stages of the evolution of low-mass stars. They are characterized by high effective temperatures, ranging from $T_{\text {eff }}=20000 \mathrm{~K}$ to more than $45000 \mathrm{~K}$, while their surface gravities are typically between $\log g=5.0$ and 6.5 (Heber 2016). The vast majority of sdB stars are helium deficient with helium abundances that can reach as low as $\log n(\mathrm{He}) / n(\mathrm{H})=-4$ (Lisker et al. 2005). Most of these stars are evolving through the core helium-burning phase on the horizontal branch but their remaining hydrogen envelope is too thin to sustain hydrogen shell burning (Dorman et al. 1993). This is why they are said to populate the extreme part of the horizontal branch (EHB) in the Hertzsprung-Russell diagram. Since the mass of sdB stars is largely dominated by that of the He-burning core (the hydrogen envelope contributes less than $2 \%$ ), the mass distribution of these stars is strongly peaked around the mass required for the He-flash $\left(\sim 0.47 M_{\odot}\right.$; Dorman et al. 1993; Han et al. 2002; Fontaine et al. 2012). Unlike normal horizontal branch objects, the sdB stars, due to their lack of $\mathrm{H}$-shell burning, evolve directly

\footnotetext{
* The averaged spectra are only available at the CDS via anonymous ftp to cdsarc.u-strasbg. fr (130.79.128.5) or via http:// cdsarc.u-strasbg.fr/viz-bin/cat/J/A+A/630/A130
}

to the white dwarf (WD) cooling sequence without an excursion to the asymptotic giant branch (AGB; Dorman et al. 1993).

The formation and evolutionary history of the sdO stars is not understood very well. Because most sdO stars are hotter and somewhat more luminous than the sdB stars, they cannot be associated to the EHB. Whether their evolution is linked to the EHB or not remains an open question. It has been suggested that He-deficient sdO stars are descendants of sdB stars, because they share the peculiar chemical composition (Heber 2016). However, the majority of sdO stars have atmospheres dominated by helium with hydrogen being a trace element only. The formation of these He-sdO stars is unlikely to be linked to the EHB and two rivaling scenarios have been invoked to explain the hydrogen deficiency, either via internal mixing (Lanz et al. 2004; Miller Bertolami et al. 2008) or via a merger of two helium white dwarfs (Zhang \& Jeffery 2012). A very small number of hot subluminous stars have atmospheres of mixed H/He composition. Because their metal content is very different from that of the extremely He-rich subdwarfs, Naslim et al. (2013) suggested to distinguish intermediate $\mathrm{H} / \mathrm{He}$ composition subdwarfs (iHesds) with $n(\mathrm{He}) / n(\mathrm{H})<4$ as a class separate from the extremely He-rich (eHe) hot subdwarfs. The ESO Supernova type Ia Progenitor surveY (SPY, Napiwotzki et al. 2019) project has provided the largest homogeneous sample of hot subdwarfs from 


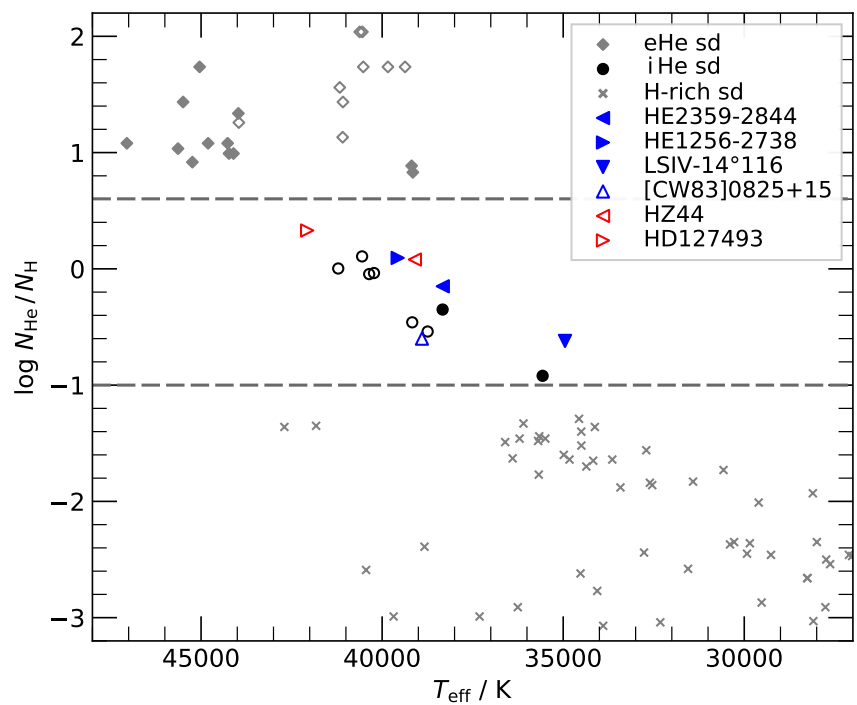

Fig. 1. Distribution of helium abundance versus $T_{\text {eff }}$ for the subdwarf population from the SPY sample (Lisker et al. 2005; Stroeer et al. 2007; Hirsch 2009). The dashed lines mark the range of iHe subdwarfs. The two heavy-metal subdwarfs from SPY (Naslim et al. 2013) and the prototypical heavy-metal subdwarfs LS IV $-14^{\circ} 116$ (Naslim et al. 2011) and [CW83] 0825+15 (Jeffery et al. 2017) are marked by blue triangles, HD 127493 and HZ 44 in red. Open symbols represent C-poor, N-rich stars, filled symbols represent C-rich stars.

high resolution spectroscopy. Through quantitative spectral analyses, $85 \mathrm{H}$-rich hot subdwarf stars have been identified, as well as $23 \mathrm{eHe}$ and $10 \mathrm{iHe}$ hot subdwarfs (Lisker et al. 2005; Stroeer et al. 2007; Hirsch 2009). The latter appear as a transition stage between the cooler H-rich sdB stars and the hotter eHe subdwarf stars (see Fig. 1). As to the carbon and nitrogen abundances, a dichotomy exists both for the eHe and the iHe sds. Stroeer et al. (2007) classified the line spectra of helium-rich hot subdwarfs into three classes: N-, C-, and C\&N-strong. Hirsch (2009) showed that, indeed, the $\mathrm{N}$ strong-lined stars are enriched in nitrogen with respect to the Sun, as are the C strong-lined stars enriched in carbon, and the C\&N strong-lined stars are enriched in both elements. This dichotomy is most obvious for the eHe hot subdwarf stars, the $\mathrm{N}$-strong ones being mostly cooler than the $\mathrm{C}$ - or $\mathrm{C} \& \mathrm{~N}$-strong ones. For the iHe hot subdwarfs such a separation is less pronounced (see Fig. 1).

Naslim et al. (2011) have discovered trans-iron elements, in particular zirconium and lead, to be strongly overabundant in the iHe-sdB LS IV $-14^{\circ} 116$. Since then, three additional intermediate He-sdBs (indicated with blue triangles in Fig. 1), with effective temperature between $35000 \mathrm{~K}$ and $40000 \mathrm{~K}$, have been found to be extremely enriched in heavy elements (Naslim et al. 2013; Jeffery et al. 2017). The origin of the extreme enrichment observed in iHe hot subdwarfs is not yet understood. Radiativelydriven diffusion has been proposed, but is poorly constrained with only four stars ([CW83] $0825+15$, LS IV $-14^{\circ} 116$, and the SPY objects HE 2359-2844 and HE 1256-2738) studied so far. Therefore, we decided to extend the sample to higher temperatures by studying HZ $44(39000 \mathrm{~K})$ and HD 127493 (42000 K) for which excellent high-resolution spectroscopy is available both for the optical and the ultraviolet spectral range. This makes them excellent targets to perform a comprehensive quantitative abundance analysis and focus on trans-iron elements.

HZ 44 and HD 127493 were among the first sdOs to be identified in the 1950s. HZ 44 was discovered in the first survey for faint blue stars in the halo by Humason \& Zwicky (1947).
The first spectral analysis of the helium line spectrum of HZ44 was published in the pioneering paper of Münch (1958). From a curve of growth analysis Peterson (1970) derived metal abundances for the first time, but we know of no contemporary study. HZ 44 is now a spectrophotometric standard star (Massey et al. 1988; Oke 1990; Landolt \& Uomoto 2007), used for the calibration of the Hubble Space Telescope (HST; Bohlin et al. 1990, 2001; Bohlin 1996), as well as that of Gaia (Marinoni et al. 2016), and therefore has frequently been observed. High resolution spectra are available from the far-UV to the red in the Far Ultraviolet Spectroscopic Explorer (FUSE), International Ultraviolet Explorer (IUE), and HIRES at Keck data archives.

HD 127493 has been used as a secondary spectrophotometric standard star (Spencer Jones 1985; Kilkenny et al. 1998; Bessell 1999). Therefore, very accurate photometry is available but spectroscopic observations are not as extensive as for HZ 44. Starting with the curve of growth analyses of Peterson (1970) and Tomley (1970), abundances of C, N, O, Ne, Mg, and Si were derived. The first non-local thermodynamic equilibrium (NLTE) model atmospheres were calculated by Kudritzki (1976), who revised the atmospheric parameters. Abundances of carbon (Gruschinske et al. 1980) and C, N, O, and Si (Simon et al. 1980) were derived from equivalent widths of ultraviolet lines. A NLTE analysis of optical spectra allowed Bauer \& Husfeld (1995) to determine the abundances of $\mathrm{C}, \mathrm{N}, \mathrm{O}, \mathrm{Ne}, \mathrm{Mg}, \mathrm{Al}$, and $\mathrm{Si}$. The most recent NLTE analysis by Hirsch (2009) revised the atmospheric parameters and determined $\mathrm{C}$ and $\mathrm{N}$ abundances from optical spectra. For completeness we give a comparison of our results with those of previous analyses in the appendix. Hence, our knowledge of the chemical composition of both stars is rather limited ${ }^{1}$.

The paper is organized as follows. In Sect. 2 we provide a description of the available spectra followed by a presentation of the atmospheric parameters that we derived for our stars in Sect. 3. The spectroscopic masses obtained from the spectral energy distributions and the Gaia parallaxes are presented in Sect. 4. The atomic data used for our abundance analysis are discussed in Sect. 5. In Sect. 6 we provide details on the abundance analysis of all considered metallic elements. The abundance patterns for HZ 44 and HD 127493 are discussed in Sect. 7 and we conclude in Sect. 8.

\section{Spectroscopic observations}

For both stars excellent archival data are available in both the optical and UV ranges. An overview of the spectra that we collected and used is given in Table 1, with additional details on the individual observations listed in Table A.1.

To determine the atmospheric parameters of HD 127493 and measure photospheric metal abundances we used optical spectra taken with the Fiber-fed Extended Range Optical Spectrograph (FEROS) that is mounted on the MPG/ESO-2.20 m telescope operated by the European Southern Observatory (ESO) in La Silla. It features a high resolving power of $R \approx 48000$ (Kaufer et al. 1999) and its usable spectral range, from $\sim 3700$ to $\sim 9200 \AA$, includes all the Balmer lines as well as many He I, He II, and metal lines. The three available spectra of HD 127493 were co-added to achieve a high signal-to-noise ratio $(\mathrm{S} / \mathrm{N})$ of $\gtrsim 100$ in the 4000-6000 $\AA$ range. Nevertheless, the S/N decreases drastically toward both ends of the spectral range and especially below $3800 \AA$.

1 The abundance analysis performed in this paper is based on, revises, and extends results for HD 127493 from Dorsch et al. (2018). 
Table 1. Spectra used for the analysis.

\begin{tabular}{llccr}
\hline \hline Star & Instrument & Range $(\AA)$ & $\mathrm{R}$ & $S / N$ \\
\hline HD 127493 & IUE SWP & $1150-1970$ & 10000 & 14 \\
& GHRS & $1225-1745$ & $0.07 \AA^{(a)}$ & 40 \\
& IUE LWR & $1850-3273$ & 10000 & 14 \\
\multirow{4}{*}{ HZ 44} & FEROS & $3700-9200$ & 48000 & 180 \\
& FUSE & $905-1188$ & 19000 & 30 \\
& IUE SWP & $1150-1970$ & 10000 & 10 \\
& HIRES & $3022-7580$ & 36000 & 142 \\
& ISIS & $3700-5260$ & $1.5 \AA^{(a)}$ & 170 \\
\hline
\end{tabular}

Notes. The signal-to-noise ratio is the average over the spectrum. ${ }^{(a)}$ The resolution for long-slit spectrographs is given instead as $\Delta \lambda$.

Both stars have been observed with the International Ultraviolet Explorer (IUE) satellite with the short-wavelength prime (SWP) camera. We retrieved three archival INES ${ }^{2}$ spectra for HD 127493 and two for HZ 44. For each star we co-added the individual spectra to increase the $\mathrm{S} / \mathrm{N}$. They continuously cover the $1150-1980 \AA$ range with a resolution of $R \approx 10000$. Additional IUE spectra taken with the long-wavelength redundant (LWR) camera (covering the 1850-3350 A range) are also available for both stars. However, these spectra have a lower quality and the S/N drops sharply at both ends of the spectra. Fewer lines are observed in this wavelength range but the IUE LWR spectrum of HD 127493 has nevertheless been useful for the abundance analysis.

HD 127493 has also been observed with the Goddard HighResolution Spectrograph (GHRS) mounted on the HST. These spectra are publicly available in the $\mathrm{MAST}^{3}$ archive and cover the $1225-1745 \AA$ range with a resolution of $\Delta \lambda \approx 0.07 \AA$. The final spectrum is a combination of ten observations spanning $35 \AA$ each and lacks coverage in the following regions: 1450.5-1532.5 ̊, 1567.7-1623.2 $\AA$, and 1658.1-1713.0 $\AA$. Since the wavelength calibration was not perfect, we cross-correlated the individual spectra to match the synthetic spectrum of HD 127493. In addition, they were shifted to match the flux level of the IUE spectra.

HZ 44 has been observed with the FUSE satellite over the spectral range between 905 and $1188 \AA$. We retrieved three calibrated observations from MAST, two taken through the low resolution (LWRS) $\left(30^{\prime \prime} \times 30^{\prime \prime}\right)$ aperture, and one through the medium resolution $($ MDRS $)\left(4^{\prime \prime} \times 20^{\prime \prime}\right)$ aperture. We co-added all spectra from the eight segments in each observation. After inspection it turned out that the MDRS spectrum had a better quality and a better wavelength calibration, so we used this spectrum for our analysis.

To determine the atmospheric parameters of HZ 44 we used a low resolution $(1.5 \AA)$, high $\mathrm{S} / \mathrm{N}$ spectrum taken with the Intermediate dispersion Spectrograph and Imaging System (ISIS) mounted at the Cassegrain focus of the $4.2 \mathrm{~m}$ William Herschel Telescope on La Palma. The spectrum covers the 3700-5260 $\AA$ range, thus including the Balmer lines, except $\mathrm{H}_{\alpha}$, as well as He I and II lines.

The spectra of HZ 44 taken with the High Resolution Echelle Spectrometer (HIRES) mounted on the Keck I telescope on

\footnotetext{
2 IUE Newly-Extracted Spectra, http://sdc.cab.inta-csic.es/ ines/index 2.html

3 Mikulski Archive for Space Telescopes, https://archive. stsci.edu/index.html
}

Mauna Kea were most valuable for our abundance analysis. A total of 68 extracted HIRES spectra of HZ 44 from several programs covering various wavelength ranges are available in the Keck Observatory Archive (KOA $)^{4}$. We co-added the spectra of four high S/N HIRES observations to produce the spectrum used for our abundance analysis. To access the ranges between 3022 and $3128 \AA$ and above $5990 \AA$ we considered two additional HIRES spectra that were used specifically for these regions. Additional spectra were also retrieved from the archive and used to measure radial velocities. Unfortunately, the normalization of HIRES spectra is difficult since the spectral orders are narrower than many broad Balmer or helium lines. This is not a problem for sharp metal lines, but renders the spectra next to useless for the determination of atmospheric parameters of HZ44.

\section{Atmospheric parameters and radial velocities}

In order to analyze the spectra of our stars we computed NLTE model atmospheres using the TLUSTY and SYNSPEC codes developed by Hubeny (1988) and Lanz \& Hubeny (2003). A detailed description of TLUSTY/SYNSPEC has recently been published in Hubeny \& Lanz (2017a,b,c).

We derived atmospheric parameters for both stars using optical spectra (besides HIRES) and a newly constructed model atmosphere grid that includes effective temperatures from $T_{\text {eff }}=$ 35000 to $48000 \mathrm{~K}$ in steps of $1000 \mathrm{~K}$, and surface gravities from $\log g=4.7$ to 6.0 in steps of 0.1 . For each of these combinations, models with helium abundances from $\log n_{\mathrm{He}} / n_{\mathrm{H}}=-1.0$ to +2.1 in steps of 0.1 were computed. All models in the grid include carbon, nitrogen, and silicon in NLTE using the abundances determined by our previous analysis of HD 127493 (Dorsch et al. 2018); this approach significantly improves the atmospheric structure compared to models that only include hydrogen and helium (e.g., Schindewolf et al. 2018). These values of C, N, and $\mathrm{Si}$ are also appropriate for HZ 44 as shown in the abundance analysis presented in Sect. 6. The selection of all lines we used, as well as the global best-fit model for HD 127493 is shown in Fig. 2. Our final best fit of the ISIS spectrum of HZ44 is shown in Fig. 3. The resulting parameters derived from the simultaneous fit of all selected $\mathrm{H}$ and He I-II lines for both stars are reported in Table 2. The atmospheric parameters for HD 127493 derived by Hirsch (2009) are also listed. They were obtained with the same FEROS spectrum but different model atmospheres and are fully consistent with our results. As shown in Fig. 1 the atmospheric parameters of both stars fit very well the trend of helium abundance to increase with increasing effective temperatures. We found no indication of rotation or microturbulence in either star; some optical metal lines are in fact sharper in the observations than in the models.

Radial velocities of HZ44 in 27 HIRES spectra taken between 1995 and 2016 were measured by Schork (2018) and are listed in Table A.2. From these values an average radial velocity of $v_{\text {rad }}=-12.7 \pm 0.4 \mathrm{~km} \mathrm{~s}^{-1}$ was derived. The measurements show that the radial velocity of HZ 44 does not vary on a scale of a few $\mathrm{km} \mathrm{s}^{-1}$. Within the radial velocity uncertainties, neither a short- nor a long-period companion is detected. Our radial velocity measurement for HD 127493 is consistent with the value derived by Hirsch (2009) using the same FEROS spectrum $\left(-17 \pm 3 \mathrm{~km} \mathrm{~s}^{-1}\right)$.

\footnotetext{
4 Keck Observatory Archive, https://koa.ipac.caltech.edu/ cgi-bin/KOA/nph-KOAlogin
} 

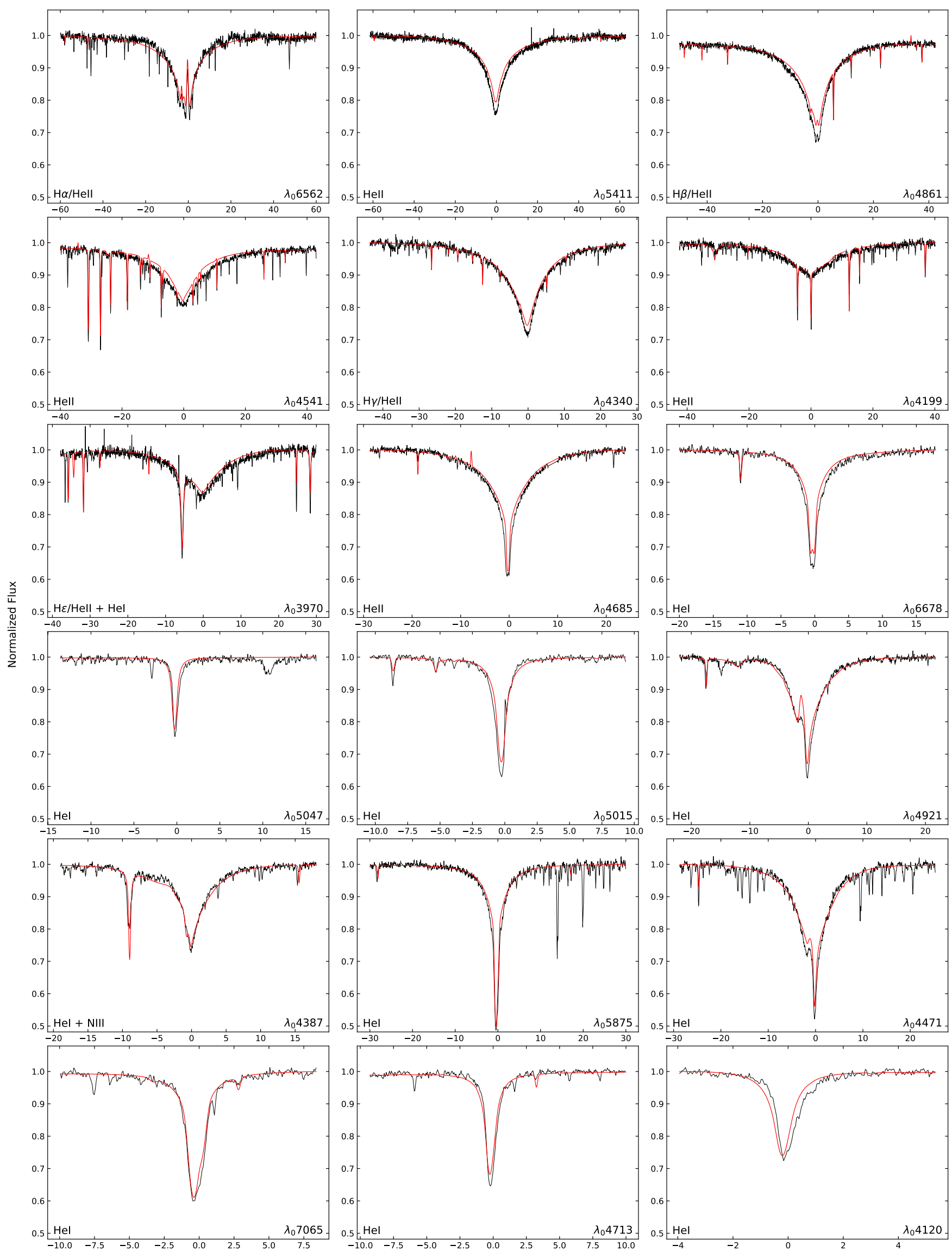

$$
\lambda-\lambda_{0}(\AA)
$$

Fig. 2. Best fit (red) to the Balmer and helium lines selected in the normalized FEROS spectrum of HD 127493 (black). 

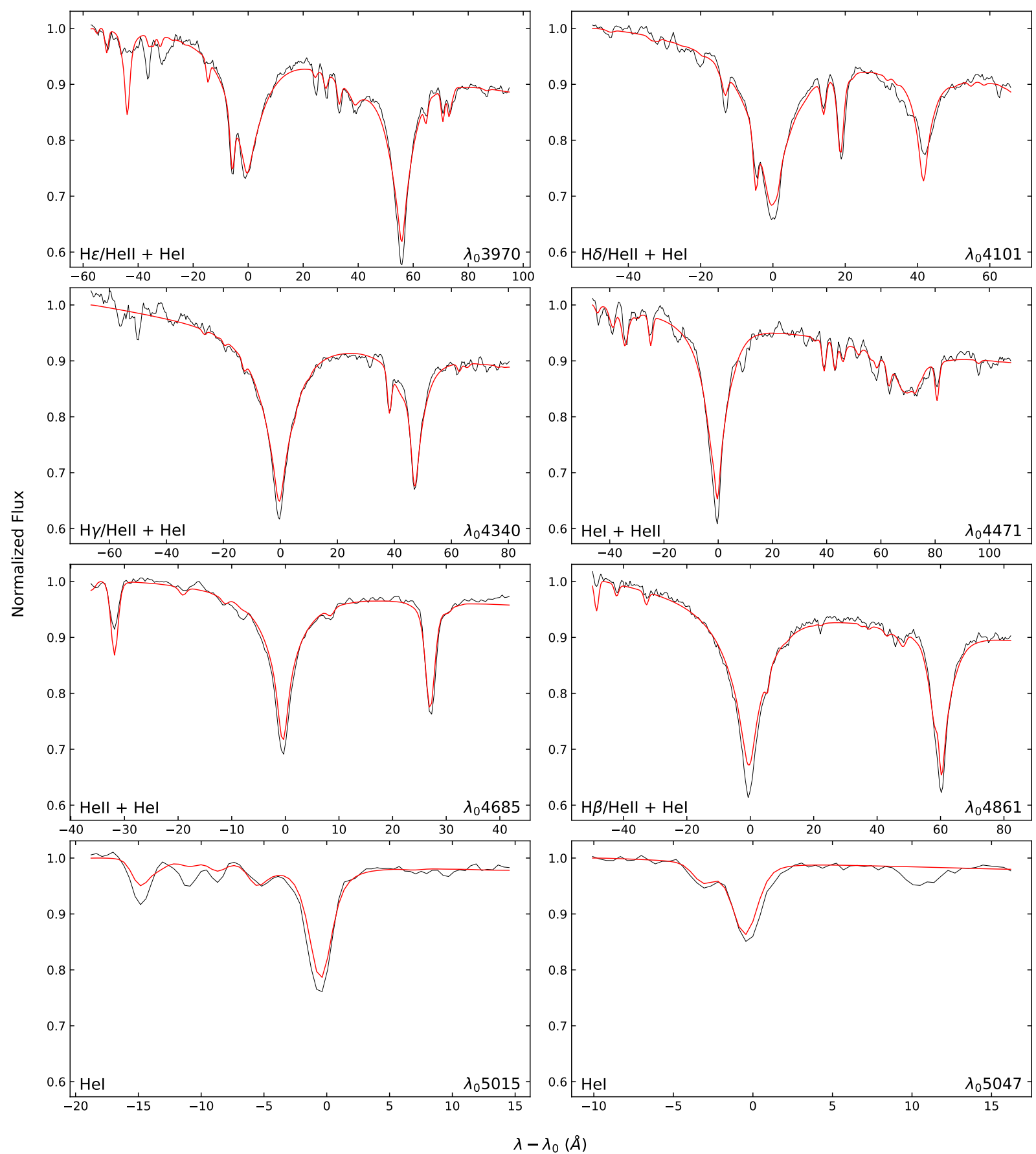

Fig. 3. Best fit (red) to the Balmer and helium lines selected in the flux-calibrated ISIS spectrum of HZ 44 (black).

Table 2. Parameters derived from optical spectroscopy.

\begin{tabular}{llccccc}
\hline \hline Name & $\begin{array}{l}T_{\text {eff }} \\
(\mathrm{K})\end{array}$ & $\begin{array}{c}\log g \\
(\mathrm{cgs})\end{array}$ & $\log n_{\mathrm{He}} / n_{\mathrm{H}}$ & $\begin{array}{c}v_{\text {rot }} \sin i \\
\left(\mathrm{~km} \mathrm{~s}^{-1}\right)\end{array}$ & Spectrum & Ref. \\
\hline HZ 44 & $39100 \pm 600$ & $5.64 \pm 0.10$ & $0.08 \pm 0.05$ & $<5$ & ISIS & 1 \\
HD 127493 & $42070 \pm 180$ & $5.61 \pm 0.04$ & $0.33 \pm 0.06$ & $<10$ & FEROS & 1 \\
& $42480 \pm 250$ & $5.60 \pm 0.05$ & $0.60 \pm 0.30$ & $<10$ & FEROS & 2 \\
\hline
\end{tabular}

Notes. The uncertainties stated were determined using different methods. Uncertainties on our results are determined using the bootstrapping method. Hirsch (2009) provide an explanation of their uncertainties.

References. ${ }^{(1)}$ This work, ${ }^{(2)}$ Hirsch (2009). 
Table 3. Parallax and parameters derived from the SED fitting.

\begin{tabular}{lcc}
\hline \hline Results & HZ 44 & HD 127493 \\
\hline$\varpi(\mathrm{mas})$ & $2.48 \pm 0.08$ & $5.82 \pm 0.09$ \\
$d(\mathrm{pc})$ & $403 \pm 13$ & $172 \pm 3$ \\
$\theta\left(10^{-11} \mathrm{rad}\right)$ & $2.134 \pm 0.011$ & $4.53 \pm 0.03$ \\
$E_{B-V}$ & $0.010 \pm 0.004$ & $0.052 \pm 0.004$ \\
$R / R_{\odot}$ & $0.191 \pm 0.007$ & $0.172 \pm 0.003$ \\
$M / M_{\odot}$ & $0.59 \pm 0.14$ & $0.43 \pm 0.05$ \\
$L / L_{\odot}$ & $77 \pm 4$ & $84 \pm 2$ \\
\hline
\end{tabular}

\section{Stellar masses, radii, and luminosities}

With the release of Gaia Data Release 2 (DR2), high accuracy parallax $(\varpi)$ and therefore distance measurements have become available for a large sample of hot subdwarfs. This allows us to derive more precise spectroscopic masses for these stars. We collected photometric measurements from several surveys and converted them into fluxes (see Tables A.3 and A.4). In addition, we used low-resolution $(\sim 6 \AA)$, large-aperture IUE spectra that were averaged in three regions (1300-1800,, $2000-2500 \AA$, 2500-3000 $\AA$ ) as "box filters" to cover the UV range. Our photometric fitting procedure is described in detail in Heber et al. (2018). The $\chi^{2}$ fitting procedure scales our final synthetic spectra to match the photometric data and has the solid angle $\theta=2 R / D$ and the color excess $E_{B-V}$ as free parameters. Reddening is modeled with $R_{V}=3.1$ as the extinction parameter (a standard value for the diffuse ISM) and the corresponding mean extinction law from Fitzpatrick (1999). The resulting solid angle can be combined with the Gaia parallax distance to obtain the stellar radius, from which the stellar mass can be computed using the surface gravity derived from spectroscopy. The SED fits are shown in Fig. 4 and the derived parameters in Table 3. Considering the non-detection of radial velocity variations and the evident lack of an IR excess, we can state that there is no indication of binarity in HZ 44. The SED of HD 127493 also shows no IR excess that would hint at a companion. The masses determined from the SED fits are consistent with the canonical subdwarf mass, $0.47 M_{\odot}$ (Fontaine et al. 2012, and references therein).

\section{Atomic data}

While atomic data and line lists for elements lighter than the iron group are readily accessible via, for example, the Kurucz compilations and the NIST ${ }^{5}$ database, data for trans-iron elements are much more scarce. Since these elements are of special interest for the analysis of our two stars, we invested particular effort into searching the literature and collecting data (energy levels, line positions, and oscillator strengths) for many trans-iron elements. We list in Table 4 the ions that we took into consideration as well as the references for their atomic data. We also include in this table, for each ion, the number of lines visible (with a predicted equivalent width greater than $5 \mathrm{~m} \AA$ ) in the final model spectrum of HZ 44. The basis of our line list is the most recent line list published by Kurucz (2018) and available online ${ }^{6}$. The list was further extended with data listed in ALL, the Atomic Line List

\footnotetext{
5 National Institute of Standards and Technology, https://physics. nist.gov/PhysRefData/ASD/lines_form.html

6 Kurucz/Linelists, http://kurucz .harvard.edu/linelists/ gfnew/gfallo8oct 17 .dat
}

(v2.05b21) ${ }^{7}$. In the context of their ongoing "Stellar Laboratories" series, Rauch et al. (2015) have published a large collection of atomic data for elements with $Z \geq 30$ on the TOSS $^{8}$ website. While this collection was made for the analysis of hot white dwarfs with $T_{\text {eff }}>60000 \mathrm{~K}$, it also includes atomic data for ions of stages IV-V that are observed in the sdOs discussed here. Thus, additional lines were added from TOSS and other theoretical works listed in Table 4. Finally the list was merged with the collection of lines from low-lying energy levels by Morton (2000) but preferring more recent data if available. Hyper-fine structure and isotopic line splitting are not considered because of the lack of atomic data. For subordinate lines the effect is expected to be small, but may be significant for resonance lines (e.g., Mashonkina et al. 2003) such as the Pb IV $1313 \AA$ A. Fortunately, for the latter resonance line atomic data are available for several isotopes and we included them in the line formation calculations (see O'Toole \& Heber 2006).

\section{Metal abundance analysis}

Model atmospheres were calculated for each star using their atmospheric parameters as listed in Table 2. All ions for which model atoms are available are included in NLTE (see Table 5), while the remaining elements are treated with the LTE approximation. The next higher ionization stage of each metal listed in Table 5 is considered as a one-level ion. More information on the model atoms we use can be found on the TLUSTY website ${ }^{9}$ and in Lanz \& Hubeny $(2003,2007)$. The Mg III-V and Ar IV-V model atoms are described in Latour et al. (2013). The Ca III-IV model atoms were constructed in a similar manner ( $\mathrm{P}$. Chayer, priv. comm.) while the Ca II model atom is described in Allende Prieto et al. (2003). To compute the partition functions of heavy elements $(Z>30)$ in ionization stages IV-VI, we added atomic data from NIST to SYNSPEC, as in Chayer et al. (2006) and Latour et al. (2019a). As a starting point, abundances in the TLUSTY model were set to values estimated by eye for each element. Based on this preliminary model, a series of synthetic spectra with a range of abundances for each element were created with SYNSPEC. The abundances of the elements were determined one by one using the downhill-simplex fitting program SPAS developed by Hirsch (2009). This method works well for isolated lines but is not reliable for heavily blended lines, in particular in the UV region. The abundance for these elements was estimated by manually comparing models with the observation. Even with this method, the placement of the continuum (especially in the FUSE range) remains an important source of uncertainty. As noted by Pereira et al. (2006), the true continuum in the FUSE spectral region may be well above the highest observed fluxes. This complicates the continuum placement since some opacity (photospheric and interstellar) is still missing in our final synthetic spectra. Thus for some elements we could only derive upper limits. This includes elements with low abundances but also elements that show lines in the FUSE range only, where the aforementioned problems are most severe. For some elements in HD 127493 no abundances, or upper limits, could be derived $(\mathrm{Cl}, \mathrm{K}, \mathrm{As}, \mathrm{Se}, \mathrm{Sb}, \mathrm{Xe}, \mathrm{Bi})$. This is due to insufficient spectral coverage: the elements in question have their strongest spectral

\footnotetext{
Atomic Line List (v2.05b21), http://www.pa.uky.edu/ peter/ newpage/

8 Tübingen Oscillator Strengths Service, http://dc.g-vo.org/ TOSS

9 http://tlusty.oca.eu/Tlusty2002/tlusty-frames-data. html
} 

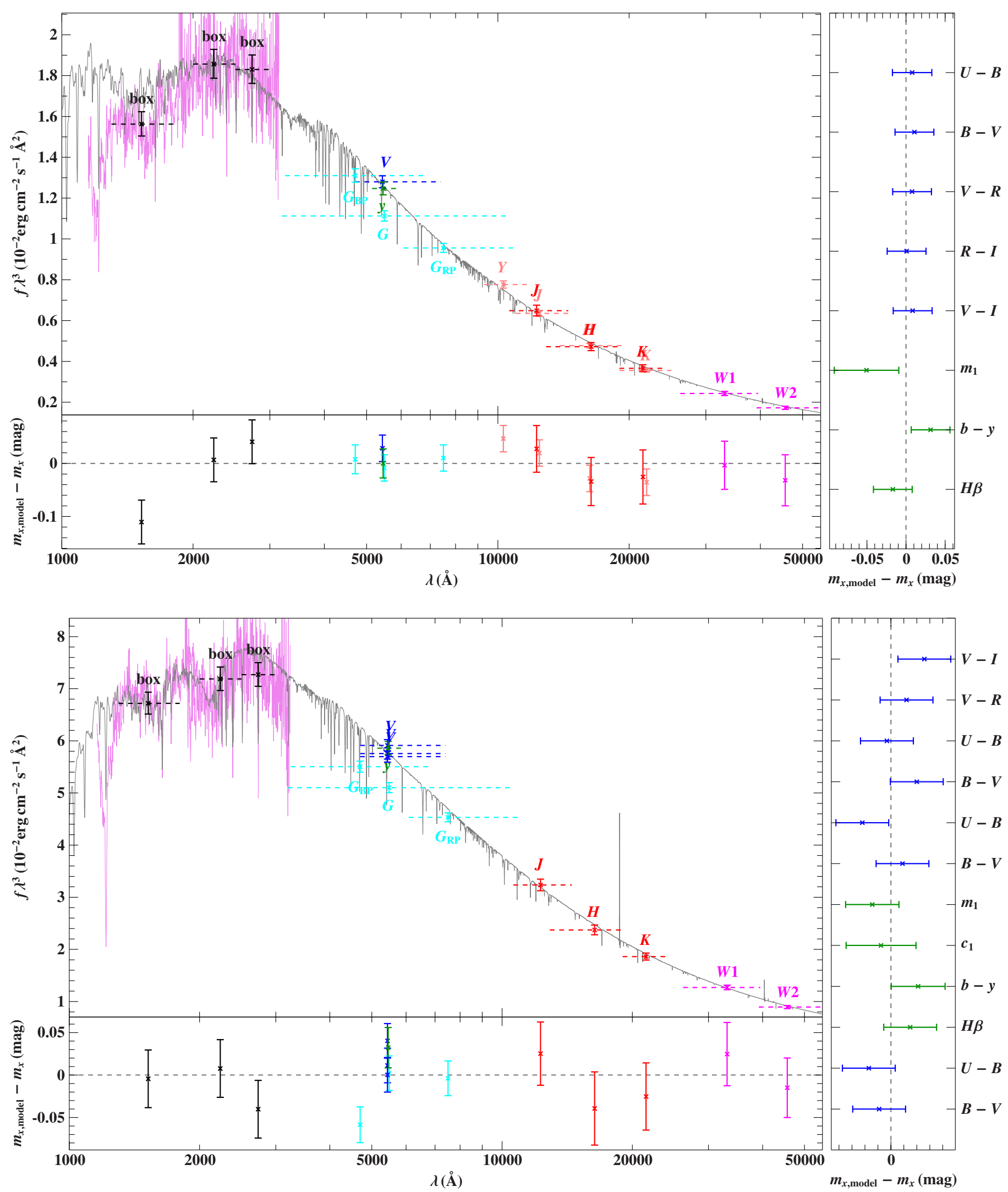

Fig. 4. Comparison of synthetic spectra with photometric data for HZ 44 (top) and HD 127493 (bottom). The three black data points labeled "box" are binned fluxes from a low-dispersion IUE spectrum. Filter-averaged fluxes are shown as colored data points that were converted from observed magnitudes (the dashed horizontal lines indicate the respective filter widths), while the gray solid line represents a synthetic spectrum using the atmospheric parameters given in Table 2. The residual panels at the bottom and right-hand side show the differences between synthetic and observed magnitudes as well as colors. The following color codes are used to identify the photometric systems: Johnson-Cousins (blue), Strömgren (green), Gaia (cyan), UKIDSS (rose), 2MASS (red), and WISE (magenta). 
Table 4. Data for elements not included in the Kurucz line-list (gfallo8oct17. dat).

\begin{tabular}{|c|c|c|c|c|c|c|c|c|c|c|c|}
\hline Ion & $N_{\mathrm{UV}}$ & $N_{\mathrm{VIS}}$ & References & Ion & $N_{\mathrm{UV}}$ & $N_{\mathrm{VIS}}$ & References & Ion & $N_{\mathrm{UV}}$ & $N_{\mathrm{VIS}}$ & References \\
\hline Ga III & 3 & 2 & $15,2(28)$ & $\mathrm{Sr} \mathrm{V}^{*}$ & 23 & - & 3 & $\mathrm{Te} \mathrm{V}^{*}$ & 1 & - & $1(30)$ \\
\hline Ga IV & 69 & - & 3 & Y III* & 1 & 2 & 10,16 & Te VI* & 2 & - & 3 \\
\hline $\mathrm{Ga} \mathrm{V}$ & 37 & - & 3 & Zr IV & 11 & 8 & 3 & $\mathrm{Xe} \mathrm{IV}^{*}$ & 5 & - & 3 \\
\hline Ge III & 1 & - & $1(25,18)$ & Zr V & - & - & 3 & $\mathrm{Xe} \mathrm{V}^{*}$ & 4 & - & 3 \\
\hline Ge IV & 7 & 6 & $23,2(28)$ & Mo IV* & 92 & - & 29,3 & $\mathrm{BaV}^{*}$ & 2 & - & 3 \\
\hline Ge V & 24 & - & 3 & Mo V* & 69 & - & 3 & Tl III* & - & - & 5 \\
\hline As III & - & - & $1(26,19,17)$ & Mo VI* & 5 & - & 3 & $\mathrm{~Pb}$ III & 2 & - & $7,1(24)$ \\
\hline As IV & - & 6 & $2(27), 1(25)$ & In III* & 5 & - & 4 & $\mathrm{~Pb}$ IV & 17 & 9 & $11,5,8,1(24)$ \\
\hline As V & 2 & - & $1(19)$ & Sn III & - & - & 13 & $\mathrm{~Pb} \mathrm{~V}$ & 36 & - & 9 \\
\hline $\mathrm{Se} \mathrm{IV}^{*}$ & 3 & - & $1(22)$ & Sn IV & 7 & - & 4,12 & Bi III* & - & - & $1(23)$ \\
\hline $\mathrm{Se} \mathrm{V}^{*}$ & 4 & - & 3 & Sb III* & - & - & $1(17)$ & Bi IV* & - & - & $1(21)$ \\
\hline $\mathrm{Kr}_{\mathrm{IV}}{ }^{*}$ & 42 & 6 & 3 & $\mathrm{Sb} \mathrm{IV}^{*}$ & 1 & - & $1(20)$ & $\mathrm{Bi} \mathrm{V}^{*}$ & 1 & - & 5 \\
\hline $\mathrm{Kr} \mathrm{V}^{*}$ & - & - & 3 & $\mathrm{Sb} \mathrm{V}^{*}$ & 2 & - & 4 & Th IV* & 2 & - & 6 \\
\hline $\mathrm{Sr}_{\mathrm{IV}}{ }^{*}$ & 109 & 1 & 3 & Te III* & - & - & 14 & & & & \\
\hline
\end{tabular}

Notes. The number of lines with predicted equivalent width greater than $5 \mathrm{~m} \AA$ in the final model of HZ 44 (upper limits marked with *) and in spectral ranges where observations are available for HZ 44 are listed (UV: $916-1980 \AA$, VIS: 3022-7580 A). For references from compilations, the compilation is listed first and the individual references in parenthesis.

References. (1) Morton (2000), (2) ALL, van Hoof (2017), (3) TOSS, Rauch et al. (2015), (4) Safronova et al. (2003), (5) Safronova \& Johnson (2004), (6) Safronova \& Safronova (2013), (7) Alonso-Medina et al. (2009), (8) Alonso-Medina et al. (2011), (9) Colón et al. (2014), (10) Naslim et al. (2011), (11) Naslim et al. (2013), (12) Biswas et al. (2018), (13) Haris \& Tauheed (2012), (14) Zhang et al. (2013), (15) Nielsen et al. (2005), (16) Redfors (1991), (17) Andersen \& Lindgard (1977), (18) Andersen et al. (1979), (19) Pinnington et al. (1981), (20) Pinnington et al. (1985a), (21) Pinnington et al. (1988), (22) Bahr et al. (1982), (23) Migdałek (1983), (24) Ansbacher et al. (1988), (25) Curtis (1992), (26) Marcinek \& Migdalek (1993), (27) Churilov \& Joshi (1996), (28) O’Reilly \& Dunne (1998), (29) Kurucz (2018), (30) Pinnington et al. (1985b).

Table 5. Model atoms used for the final model of HZ44 with their number of explicit levels (L) and superlevels (SL).

\begin{tabular}{lrrllrr}
\hline \hline Ion & L & SL & & Ion & L & SL \\
\cline { 1 - 2 } H I & 17 & - & & Si II & 36 & 4 \\
He I & 24 & - & & Si III & 31 & 15 \\
He II & 20 & - & Si IV & 19 & 4 \\
C II & 34 & 5 & P IV & 14 & - \\
C III & 34 & 12 & P V & 13 & 4 \\
C IV & 35 & 2 & S II & 23 & 10 \\
N II & 32 & 10 & S III & 29 & 12 \\
N III & 40 & 9 & S IV & 33 & 5 \\
N IV & 34 & 14 & & S V & 20 & 5 \\
N V & 21 & 4 & & S VI & 13 & 3 \\
O II & 36 & 12 & & Ar II & 42 & 12 \\
O III & 28 & 13 & & Ar III & 27 & 17 \\
O IV & 31 & 8 & Ar IV & 39 & - \\
O V & 34 & 6 & Ar V & 25 & - \\
O VI & 15 & 5 & Ca II & 32 & - \\
Ne II & 23 & 9 & Ca III & 15 & 4 \\
Ne III & 22 & 12 & Ca IV & 17 & 4 \\
Ne IV & 10 & 2 & & Fe III & - & 50 \\
Mg II & 21 & 4 & Fe IV & - & 43 \\
Mg III & 37 & 3 & Fe V & - & 42 \\
Mg IV & 29 & 5 & Ni III & - & 36 \\
Mg V & 18 & 2 & Ni IV & - & 38 \\
Al II & 20 & 9 & & Ni V & - & 48 \\
Al III & 19 & 4 & & Total & 1062 & 506 \\
\hline & & & & & &
\end{tabular}

lines in ranges where no data are available for that star (FUSE, $3000-3700 \AA)$.

A summary of the photospheric abundances derived for HZ 44 and HD 127493 is presented at the end of this section in
Fig. 12 and in Table A.5. In addition, we include in Appendix A.3 a comparison between the final synthetic spectrum and the observed spectrum in all wavelength ranges for both stars. We note that for some elements, namely $\mathrm{Ne}, \mathrm{Ar}, \mathrm{Cl}, \mathrm{Sn}, \mathrm{Tl}, \mathrm{Pb}$, and Th, the uncertainty on their solar photospheric abundance (Asplund et al. 2009) contributes significantly to the total uncertainty when computing the ratio with solar abundances. The uncertainty stated on upper limits and by-eye abundances is defined as follows: at the upper bound the lines are judged to be clearly too strong, while they cannot be distinguished from noise at the lower bound.

In the following subsections, we present in detail the result of our abundance analysis for each element. Light elements $(\mathrm{C}, \mathrm{N}$, $\mathrm{O}$ ) are discussed in Sect. 6.1, intermediate elements (F, Ne, Na, $\mathrm{Mg}, \mathrm{Al}, \mathrm{Si}, \mathrm{P}, \mathrm{S}, \mathrm{Cl}, \mathrm{Ar}, \mathrm{K}, \mathrm{Ca}, \mathrm{Ti}$ ) in Sect. 6.2, iron-group elements treated in NLTE (Fe, Ni) in Sect. 6.3, iron-group elements treated in LTE (V, Cr, Mn, Co, Cu, Zn) in Sect. 6.4, detected trans-iron elements $(\mathrm{Ga}, \mathrm{Ge}, \mathrm{As}, \mathrm{Se}, \mathrm{Zr}, \mathrm{Sn}, \mathrm{Pb})$ in Sect. 6.5, and trans-iron elements with upper limits $(\mathrm{Kr}, \mathrm{Sr}, \mathrm{Y}, \mathrm{Mo}, \mathrm{Sb}, \mathrm{Te}$, $\mathrm{Xe}, \mathrm{Th})$ in Sect. 6.6. Finally, Sect. 6.7 addresses the elements for which we could not even assess an upper limit due to the weakness of their predicted lines ( $\mathrm{Sc}, \mathrm{In}, \mathrm{Ba}, \mathrm{Tl}, \mathrm{Bi}$ ). We then discuss the chemical portrait obtained for both stars in Sect. 6.8. In the rest of the paper we give our abundances as $\log n_{X} / n_{H}$ and use the shorter notation $\log X / H$. Here, $n_{X}$ is the dimensionless number fraction. To put the abundances into perspective, we additionally state the corresponding number fraction relative to solar values $n_{X} / n_{X, \odot}$.

\subsection{Light metals $(C, N, O)$}

The carbon abundance in HZ 44 was measured using nine optical $\mathrm{C}$ III lines. The abundance derived this way, $\log \mathrm{C} / \mathrm{H}=$ $-4.31 \pm 0.13\left(8.6 \times 10^{-2}\right.$ times solar $)$, is consistent with the strong C III and CIV lines observed in the UV region. Carbon lines are weaker in the optical spectrum of HD 127493. We 
use the resonance doublet $\mathrm{C}$ IV $\lambda \lambda 1548,1551 \AA$ and the $\mathrm{C}$ III sextuplet lines at $1175 \AA$ to derive an abundance of $\log \mathrm{C} / \mathrm{H}=$ $-4.30 \pm 0.08\left(3.9 \times 10^{-2}\right.$ times solar $)$.

The nitrogen abundances measured in HZ 44 from different ionization stages and lines in the optical region are not very consistent. Most N III lines are well reproduced; some are too strong (e.g., N III $\lambda \lambda 4378.99,4379.20 \AA$ ) while a few are too weak (e.g., N III 4003.58 ). We identified only one strong N IV line in the optical spectrum of HZ 44 (N IV $4057.76 \AA$ ) that fits the final model well. We measure an abundance of $\log N / H=$ $-2.39 \pm 0.21$ (29 times solar) for HZ 44. However, many strong $\mathrm{N}$ II lines are too broad and shallow in the model, even assuming a rotation velocity and microturbulence of $0 \mathrm{~km} \mathrm{~s}^{-1}$ and were therefore excluded from the fit (e.g., N II $\lambda \lambda 3995.00,4041.31$, $4236.91 \AA$ ). This may be related to numerical issues due to low population numbers. In HD 127493 the issues with some optical $\mathrm{N}$ II lines are even more pronounced; they appear in emission in the model and were excluded from the fit. In addition to optical N III-IV lines we used UV lines to constrain the abundance, including the $\mathrm{N} v \lambda \lambda 1238.8,1242.8 \AA$ resonance lines, the strong N IV $1718 \AA$ line, and several N III lines. All ionization stages give a consistent abundance of $\log \mathrm{N} / \mathrm{H}=-2.09 \pm 0.17$ (26 times solar).

The abundance of oxygen in HZ 44 was measured using optical O II and O III lines. Although these lines are weak compared to lines from other elements, they could be used to find an abundance of $\log \mathrm{O} / \mathrm{H}=-3.77 \pm 0.13\left(12 \times 10^{-2}\right.$ times solar $)$. The strongest observed O lines in the UV region (O III $1150.884 \AA$ in FUSE and O IV $1343.51 \AA$ in the IUE/SWP spectrum) support this value. The O IV $\lambda \lambda 1338.62,1343.51 \AA$ lines are observed in the GHRS spectrum of HD 127493 but blended with Ni lines. Since no optical lines were detected in that star we only set an upper limit of $\log \mathrm{O} / \mathrm{H} \leq-4.3\left(2 \times 10^{-2}\right.$ times solar $)$ and note that the actual abundance is likely to be not significantly lower.

\subsection{Intermediate metals (F to Ti)}

For $\mathrm{HZ} 44$, all elements from fluorine to titanium were analyzed. Due to the lack of UVA and FUSE spectra, P, Cl, Ar, K, and Ti could not be studied in HD 127493.

No fluorine lines are observed in HZ44 which allows us to provide an upper limit of $\log \mathrm{F} / \mathrm{H} \leq-5.0 \pm 0.4$ (130 times solar) based on F II $\lambda \lambda 3503.11 ， 3505.63 ， 3847.09 ， 3849.99$, and $3851.67 \AA$. None of these F II lines are strong enough in HD 127493 to set a meaningful limit on the abundance.

The neon abundance measurement for HZ 44 is based on several strong Ne II lines, which are weaker in HD 127493 due to its higher temperature. Most of them lie between 3300 and $3800 \AA$ though some strong Ne II lines exist at longer wavelengths. Fitting all accessible $\mathrm{Ne}$ lines results in $\log \mathrm{Ne} / \mathrm{H}=-3.23 \pm 0.11$ (3.3 times solar) for $\mathrm{HZ} 44$ and $\log \mathrm{Ne} / \mathrm{H}=-2.38 \pm 0.18$ (3.1 times solar) for HD 127493. The weaker Ne III lines are reasonably well reproduced with the abundance stated above.

Sodium lines are weak, but clearly visible in HZ 44, most notably NaII $\lambda \lambda$ 3285.61, 3533.06, 3631.27, 4392.81 ̊. We find $\log \mathrm{Na} / \mathrm{H}=-4.43 \pm 0.06$ (10 times solar) for $\mathrm{HZ} 44$. The Na II $\lambda \lambda$ 4392.81, 4405.12 $\AA$ lines allow an upper limit of $\log \mathrm{Na} / \mathrm{H} \leq-3.4 \pm 0.3$ (48 times solar) to be set for HD 127493 .

The strongest observed magnesium lines are by far the $\mathrm{Mg}$ II triplet at $4481 \AA$. All other optical lines are too weak to be observed in either star. We derive $\log \mathrm{Mg} / \mathrm{H}=-3.8 \pm 0.2$ (1.9 times solar) for $\mathrm{HZ} 44$ and $\log \mathrm{Mg} / \mathrm{H}=-3.4 \pm 0.2$ (2.1 times solar) for HD 127493, consistent with Mg II $2798.82 \AA$ in the IUE LWR spectrum.

We measure the aluminum abundance in $\mathrm{HZ} 44$ to be $\log \mathrm{Al} / \mathrm{H}=-4.86 \pm 0.11$ (2.4 times solar) based on 11 optical Al III lines. This abundance is consistent with strong Al III lines observed in the UV range, including the Al III $\lambda \lambda$ 1854.72, $1862.79 \AA$ resonance lines. The Al abundance in HD 127493 , $\log \mathrm{Al} / \mathrm{H}=-4.53 \pm 0.10$ (2.2 times solar), is derived from Al III $\lambda \lambda 4479.97,4512.57,4529.19,5696.60 \AA$.

Both stars show strong silicon lines in their optical and UV spectra. The abundance measurement for HZ 44 is based on ten optical Si III and nine optical Si IV lines. The derived abundance of $\log \mathrm{Si} / \mathrm{H}=-3.88 \pm 0.11$ (2.0 times solar) is consistent with the resonance lines Si IV $\lambda \lambda 1394,1403 \AA$, the very strong Si IV $\lambda \lambda$ 1066.6, 1122.5, $1128.3 \AA$, and Si III 1113.2 $\AA$ in the FUSE spectrum, as well as many more silicon lines in the UV region. For HD 127493 we used three lines in the UV (including Si IV $\lambda \lambda 1394,1403 \AA$ ) for our fit in addition to four lines from the optical range. Both UV and optical lines give a consistent abundance of $\log \mathrm{Si} / \mathrm{H}=-3.31 \pm 0.14$ (3.2 times solar).

The strongest observed phosphorus lines lie in the FUSE spectral range, which is only accessible for $\mathrm{HZ} 44$. This includes the P V $\lambda \lambda 1117.98,1128.01 \AA$, and P IV 950.66 $\AA$ resonance lines as well as several strong P IV lines, such as P IV 1030.52 $\AA$. In the optical and UVA (here: $3000-3700 \AA$ ) ranges, there are only three observable lines: P IV $\lambda \lambda$ 3347.74, 3364.47 $\AA$ (see Fig. 5), and P IV $4249.66 \AA$. We derive an abundance of $\log \mathrm{P} / \mathrm{H}=$ $-5.66 \pm 0.25$ (4.1 times solar) for HZ 44. There are only two unambiguously identified P lines in HD 127493: P IV 1888.52 ̊ and P IV $4249.66 \AA$. They can be used to derive an abundance of $\log \mathrm{P} / \mathrm{H}=-4.7 \pm 0.4$ (16 times solar).

Besides many strong sulfur III-IV lines at optical wavelengths, we observed strong S III-VI lines in the UV spectrum of HZ 44 (e.g., S IV $\lambda \lambda$ 1062.66, 1072.97, 1623.94 ̊). However, some of these lines are listed with inaccurate atomic data in the newest Kurucz line list, in which case we preferred older values. The abundance measurement of $\log \mathrm{S} / \mathrm{H}=-3.87 \pm 0.37$ (4.9 times solar) based on optical S III-IV lines is consistent with the UV lines. Several optical S III lines are too weak and broad in the model (e.g., S III $\lambda \lambda$ 3497.28, 3662.01, 3717.77, $3928.61,4361.47 \AA$ ) and were excluded from the fit. Sulfur lines are slightly weaker in HD 127493 . We derive an abundance of $\log \mathrm{S} / \mathrm{H}=-3.90 \pm 0.35$ (2.0 times solar) from UV lines, consistent with optical lines such as S IV $\lambda \lambda$ 4485.64, 4504.11, 5497.78 A.

Chlorine shows strong lines from low-lying levels in the FUSE spectral region (Cl IV $\lambda \lambda$ 973.22, 977.57, 977.90, 984.96, $985.76 \AA$ ). Although these lines are strong in HZ 44, it is hard to determine abundances from them as they are rather insensitive to changes in abundance due to saturation effects. In addition, the usual problems with lines in the FUSE spectra apply: they suffer from unidentified blends, both of stellar and interstellar origin. Nevertheless, the abundance of $\log \mathrm{Cl} / \mathrm{H}=-7.0 \pm 0.7$ (0.05 times solar) derived from these lines is remarkably low despite the large uncertainty.

Argon shows many strong lines in the UVA spectrum of $\mathrm{HZ} 44$. We determine an abundance of $\log \mathrm{Ar} / \mathrm{H}=-3.79 \pm 0.11$ (31 times solar) for HZ 44 based on optical/UVA lines. Some strong Ar III lines (e.g., Ar III $\lambda \lambda 3286.11,3302.19,3311.56 \AA$ ) were excluded from the fit since they show the same discrepancies already observed in N II lines - they are very narrow in the observation and too broad in the model. Except for a very 


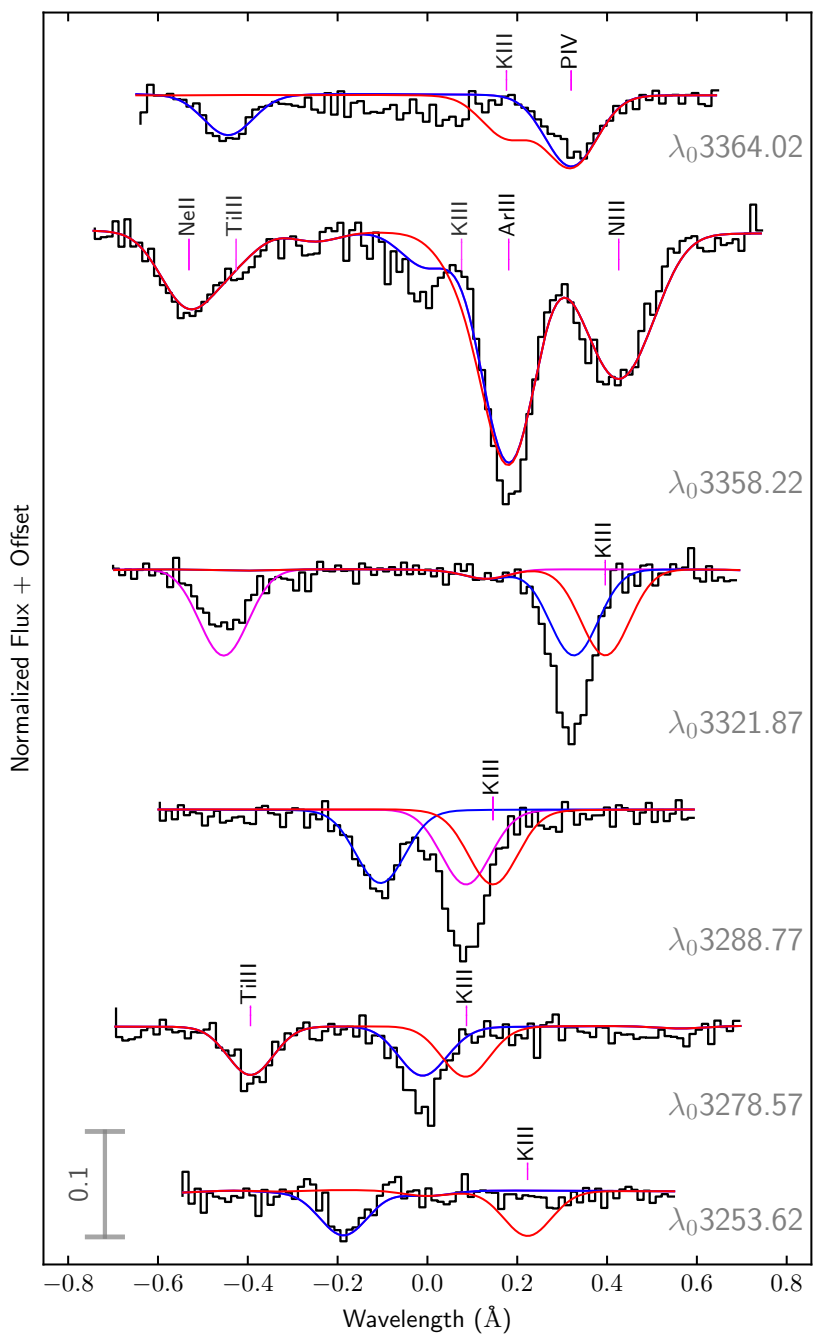

Fig. 5. Strongest K III lines in the observed spectrum of HZ44. The model using the Kurucz wavelengths is shown in red, while a model with shifted lines is shown in blue. Alternative line shifts are shown in violet (marked with ${ }^{*}$ in Table 6). All models have a potassium abundance of $\log \mathrm{K} / \mathrm{H}=-5$.

weak Ar III $4182.97 \AA$ A line, we could identify no optical Ar lines in HD 127493. The upper limit for HD 127493 derived from this line is still super-solar at $\log \mathrm{Ar} / \mathrm{H} \leq-3.6 \pm 0.2$ (21 times solar). The blended Ar IV $\lambda \lambda$ 1409.30, 1435.56 $\AA$, and Ar IV 2641.09 are well reproduced at this abundance but ArV $1371.87 \AA$ suggests a lower abundance.

We found no strong potassium lines in the UV spectrum of HZ 44, but some optical lines were clearly identified. However, several lines appear to lie at shorter wavelengths than listed in the Kurucz line list. Since the difference correlates with their LS-coupling terms, it seemed reasonable to shift them in order to match their observed position. Their wavelengths and configurations are listed in Table 6 . Other $\mathrm{K}$ lines are clearly identified at wavelengths very close to their listed value (K III $\lambda \lambda 3052.016$, 3468.314, 3513.822 ̊). As shown in Fig. 5, lines with a ${ }^{4} \mathrm{P}$ lower term had to be shifted by approximately $-0.1 \AA$, whereas the shift was larger for all lines with ${ }^{2} \mathrm{P}$ lower terms. All identified lines are reproduced reasonably well with an abundance of $\log \mathrm{K} / \mathrm{H}=-4.92 \pm 0.16$ (55 times solar), when shifted to the observed position.

While there are no usable calcium lines in the optical spectrum of HD 127493, HZ44 shows some strong Ca II and
Table 6. K III lines with deviation between predicted and observed wavelengths.

\begin{tabular}{lllc}
\hline \hline$\lambda_{\text {Kurucz }}(\AA)$ & $\lambda_{\text {obs }}(\AA)$ & $\Delta \lambda(\AA)$ & Configuration \\
\hline 3253.973 & 3253.563 & -0.41 & $4 \mathrm{~s}^{2} \mathrm{P}_{3 / 2}-4 \mathrm{p}^{2} \mathrm{D}_{3 / 2}^{\circ}$ \\
3278.787 & 3278.687 & -0.10 & $4 \mathrm{~s}^{4} \mathrm{P}_{5 / 2}-4 \mathrm{p}^{4} \mathrm{P}_{3 / 2}^{\circ}$ \\
3289.046 & 3288.796 & -0.25 & $4 \mathrm{~s}^{2} \mathrm{P}_{3 / 2}-4 \mathrm{p}^{2} \mathrm{D}_{5 / 2}^{\circ}$ \\
& $3288.9866^{*}$ & -0.06 & \\
3322.396 & 3322.326 & -0.07 & $4 \mathrm{~s}^{4} \mathrm{P}_{5 / 2}-4 \mathrm{p}^{4} \mathrm{P}_{5 / 2}^{\circ}$ \\
& 3321.546 & -0.85 & \\
3358.426 & 3358.346 & -0.08 & $4 \mathrm{~s}^{4} \mathrm{P}_{3 / 2}-4 \mathrm{p}^{4} \mathrm{P}_{1 / 2}^{\circ}$ \\
3364.326 & 3363.706 & -0.62 & $4 \mathrm{~s}^{2} \mathrm{P}_{1 / 2}-4 \mathrm{p}^{2} \mathrm{P}_{3 / 2}^{\circ}$ \\
3468.314 & 3468.260 & -0.05 & $4 \mathrm{~s}^{4} \mathrm{P}_{3 / 2}-4 \mathrm{p}^{4} \mathrm{P}_{5 / 2}^{\circ}$ \\
3513.822 & 3513.782 & -0.04 & $4 \mathrm{~s}^{4} \mathrm{P}_{1 / 2}-4 \mathrm{p}^{4} \mathrm{P}_{3 / 2}^{\circ}$ \\
\hline
\end{tabular}

Notes. The configurations are taken from NIST. The superscript ${ }^{\circ}$ indicates odd parity. The superscript ${ }^{*}$ marks alternative identifications.

Ca III lines. The optical resonance lines Ca II $\lambda \lambda 3934,3968 \AA$ are almost entirely photospheric. Some strong $\mathrm{Ca}$ III lines were excluded from the fit (e.g., Ca III $\lambda \lambda 3372.68,3537.78 \AA$ ) since they have sharp cores and are too broad and shallow in the model. NLTE effects cannot be blamed since we included Ca II and $\mathrm{Ca}$ III in NLTE. We measure $\log \mathrm{Ca} / \mathrm{H}=-3.9 \pm 0.24$ (28 times solar) for HZ44 and derive an upper limit of $\log \mathrm{Ca} / \mathrm{H} \leq$ $-3.9 \pm 0.2$ (12 times solar) for HD 127493. This upper limit is likely to be close to the actual abundance since including $\mathrm{Ca}$ at this abundance improves the fit for blended UV lines such as Ca III 1545.30 ̊ and Ca IV $\lambda \lambda$ 1647.44, 1648.62, 1655.53.

The strongest titanium III-IV lines lie in the UVA spectral region although some lines exist at longer wavelengths. We measure a strong enrichment in $\mathrm{HZ} 44$ with $\log \mathrm{Ti} / \mathrm{H}=-4.56 \pm 0.22$ (150 times solar). This abundance is consistent between strong optical and ultraviolet lines (e.g., Ti IV $\lambda \lambda$ 1183.63, 1451.74, 1467.34, $1469.19 \AA$, and Ti III $1498.70 \AA$ ). Although Ti lines at wavelengths above $3800 \AA$ are strong in HZ 44, the same lines are weak in HD 127493. The few lines that can clearly be identified in HD 127493 (Ti IV $\lambda \lambda 4397.31,5885.97 \AA$ ) do not give a consistent abundance. Other predicted lines (Ti IV $\lambda \lambda 4397.31$, $5398.93,5492.51 \AA$ A ) are not observed. Therefore we adopt a conservative upper limit of $\log \mathrm{Ti} / \mathrm{H} \leq-4.40 \pm 0.25$ (94 times solar) for HD 127493 based on optical lines. However, Ti III-IV lines in the IUE range would favor higher abundances.

\subsection{Fe and Ni (NLTE)}

We determine iron abundances by fitting the IUE spectrum of HZ44 and the GHRS spectrum of HD 127493 in ranges that span 10-20 ̊, from $1300 \AA$ onward (at shorter wavelengths, the amount of unidentified opacity increases). Since $\mathrm{Fe}$ and Ni were fitted separately, blends are not treated exactly, which may lead to overestimated abundances. However, since abundances in the initial model were already close to the best-fit abundances, this effect is partly compensated for. Missing opacity from other sources may also introduce a bias toward higher abundances but since the observed spectrum is well reproduced in the considered ranges, we are confident that the derived abundances are reliable within their respective uncertainties. The average of the abundances over all ranges yields $\log \mathrm{Fe} / \mathrm{H}=-4.00 \pm 0.25$ (1.5 times solar) for $\mathrm{HZ} 44$ and $\log \mathrm{Fe} / \mathrm{H}=-2.82 \pm 0.13$ (10 times solar) 


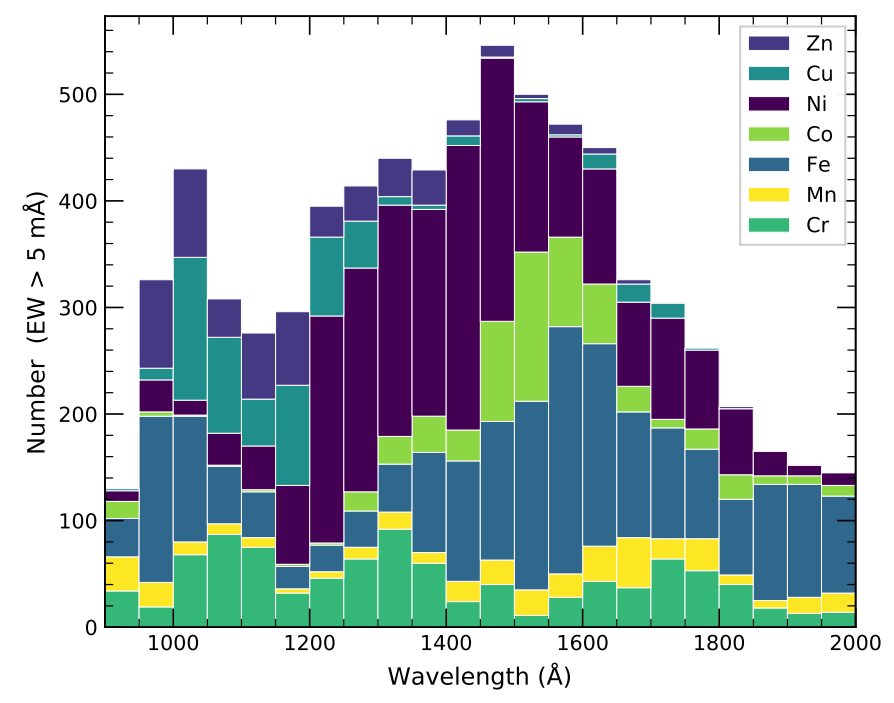

Fig. 6. Histogram of iron-peak lines with estimated equivalent widths larger than $5 \mathrm{~m} \AA$ in the final model of HZ 44. Bins are every $50 \AA$. Elements are listed in the legend in the same order as they appear in the histogram.

for HD 127493. The same procedure was applied for nickel, resulting in $\log \mathrm{Ni} / \mathrm{H}=-4.05 \pm 0.15$ (26 times solar) for $\mathrm{HZ} 44$ and $\log \mathrm{Ni} / \mathrm{H}=-3.61 \pm 0.13$ (31 times solar) for HD 127493 .

\subsection{Additional iron-group abundances (LTE)}

The UV spectral range is dominated by lines from iron-peak elements. Although most lines are from iron and nickel, opacity contributions from other iron-peak elements are also significant. Figure 6 shows the number of lines from iron-peak elements with estimated equivalent width larger than $5 \mathrm{~m} \AA$ in the final model for HZ 44. While many of these lines are observed in FUSE and IUE spectra, the opacity peak below $900 \AA$ is outside of our observed spectral range. Our models include only $\mathrm{Fe}$ and $\mathrm{Ni}$ in NLTE.

Many vanadium lines in the IUE spectrum of HZ 44 would fit well with abundances of up to $\log \mathrm{V} / \mathrm{H}=-4.8$ (e.g., V IV $\lambda \lambda 1226.53,1308.05,1356.53,1426.65,1520.16$, 1522.51, 1810.58, 1817.69, $1861.57 \AA$, and V V 1680.20 ̊). It seems unlikely that such a large amount of lines would fit the observation due to accidental alignment with unmodeled blends. However, other lines suggest abundances below $\log \mathrm{V} / \mathrm{H} \leq$ $-6.0 \pm 0.4$, for example V IV $\lambda \lambda 1317.56,1329.28,1355.13$, $1806.20 \AA$. Several lines in the FUSE spectrum seem to exclude abundances of more than $\log \mathrm{V} / \mathrm{H} \leq-5.2 \pm 0.4$, for example V IV $\lambda \lambda 1071.06,1112.20,1123.43 \AA$, and V III 1149.95 although they lie in regions where the continuum is poorly defined. We conclude that a precise abundance determination for vanadium would require a more complete model, possibly including $\mathrm{V}$ in NLTE. Alternatively, it is possible that some $\mathrm{V}$ oscillator strengths are uncertain. Therefore we set an upper limit of $\log \mathrm{V} / \mathrm{H} \leq-4.8 \pm 0.4$ (893 times solar) for $\mathrm{HZ} 44$. As for HD 127493, V IV $\lambda \lambda 1317.56,1329.28 \AA$ seem to exclude abundances higher than $\log \mathrm{V} / \mathrm{H} \leq-6.0 \pm 0.4$ (25 times solar). However, these lines may not be reliable since they also give a low upper limit in HZ 44. We therefore adopt no upper limit for HD 127493.

Chromium shows many strong lines in the ultraviolet spectrum of both stars, for example CrIV $\lambda \lambda 1433.89,1658.08$, $1825.00,1826.22,1826.88,1827.43 \AA$. The overall fit is good and we adopt an abundance of $\log \mathrm{Cr} / \mathrm{H}=-4.4 \pm 0.3$ (28 times solar) for $\mathrm{HZ} 44$ and $\log \mathrm{Cr} / \mathrm{H}=-3.9 \pm 0.3$ (76 times solar) for HD 127493.

We derive the manganese abundance in HZ 44 from FUSE and IUE to be $\log \mathrm{Mn} / \mathrm{H}=-4.9 \pm 0.4$ (22 times solar). Fairly strong and unblended lines are, among many others: Mn III $\lambda \lambda$ 917.80, 956.47 $\AA$ and Mn IV $\lambda \lambda$ 1450.36, 1780.00, $1786.05 \AA$. For HD 127493 we derive an upper limit of $\log \mathrm{Mn} / \mathrm{H} \leq-5.5 \pm 0.3$ (2.5 times solar) from several undetected lines in the GHRS spectrum such as Mn IV $\lambda \lambda 1244.33,1720.87$, $1721.49,1724.90 \AA$.

Cobalt lines in the FUSE spectrum of HZ44 (e.g., Co III $\lambda \lambda$ 944.77, 946.54, 946.61 $̊$ ) suggest an upper limit of $\log \mathrm{Co} / \mathrm{H} \leq-5.6 \pm 0.4$. Many Co lines in the IUE region, such as Co IV $\lambda \lambda 1451.43,1502.06,1502.70,1508.42 \AA$, support this upper limit. Other lines fit well with this upper limit or a slightly higher abundance: Co IV $\lambda \lambda$ 1494.75, 1500.58, 1502.19, $1565.91 \AA$. Because of the unambiguous identification of Co lines and the slight discrepancy between upper limit and best fit, we adopt $\log \mathrm{Co} / \mathrm{H}=-5.6 \pm 0.5$ (12 times solar) with a relatively large uncertainty. In HD 127493, Co IV $\lambda \lambda 1535.28$, 1540.56, 1548.83, 1559.64, 1636.40 $⿱$ are resolved by GHRS and fit well with an abundance of $\log \mathrm{Co} / \mathrm{H}=-5.1$, while Co IV $\lambda \lambda 1415.05,1550.28,1562.06 \AA$ suggest an abundance no higher than $\log \mathrm{Co} / \mathrm{H} \leq-5.3$. We therefore adopt an abundance of $\log \mathrm{Co} / \mathrm{H}=-5.3 \pm 0.3$ (9 times solar) and note that discrepancies between single lines could result from inaccuracy in line wavelengths or NLTE effects.

Many strong copper lines lie in the FUV spectral region (here: 900-1190 $\AA$ ). The Cu IV $\lambda \lambda$ 1053.73, $1057.62 \AA$ lines in the FUSE spectrum of HZ 44 are quite strong and almost free from blends. Other strong $\mathrm{Cu}$ lines are affected by unidentified blends or lie in a region where the continuum placement is not well constrained. $\mathrm{Cu}$ lines are weaker in the IUE spectrum, with a few notable exceptions: $\mathrm{Cu}$ III $\lambda \lambda$ 1674.59, 1684.63, 1702.11 $1709.03,1722.37 \AA$. We determine an abundance of $\log \mathrm{Cu} / \mathrm{H}=$ $-5.8 \pm 0.4$ (49 times solar) from the lines listed above. $\mathrm{Cu} \mathrm{V}$ lines such as $\mathrm{Cu}$ V $\lambda \lambda 1245.99,1255.30,1268.32,1274.74,1286.55$, $1299.16 \AA$ in the GHRS spectrum of HD 127493 exclude abundances higher than $\log \mathrm{Cu} / \mathrm{H} \leq-6.1 \pm 0.4$ (11 times solar).

The zinc abundances in HZ 44 and HD 127493 are based on strong Zn III-IV lines that lie mostly in the IUE spectral range. $\mathrm{ZnIV}$ is the dominant ion in HD 127493 while HZ 44 shows about the same amount of $\mathrm{Zn} \mathrm{III}$ and $\mathrm{ZnIV}$ lines. We derive $\log \mathrm{Zn} / \mathrm{H}=-5.7 \pm 0.3$ (26 times solar) for $\mathrm{HZ} 44$ and $\log \mathrm{Zn} / \mathrm{H}=-5.3 \pm 0.3$ (29 times solar) for HD 127493 .

\subsection{Detected trans-iron peak elements (LTE)}

We were able to measure the abundance of $\mathrm{Ge}, \mathrm{Ga}$, and $\mathrm{Pb}$ based on their UV lines in both HZ 44 and HD 127493. In HZ 44 we could additionally derive abundances for As and Sn based on the FUSE spectrum.

In the following we will give a brief overview of the atomic data and lines used for the abundance measurement of each element. The uncertainties on the abundances can be quite large. This could be the result of strong blending with unidentified lines, of the sparse atomic data available for most of these elements, and potential NLTE effects. Even if atomic data are available, oscillator strengths and line wavelengths are not always well tested.

We use data from TOSS for gallium IV-V and data from O'Reilly \& Dunne (1998) for Ga III, with updates for two lines 


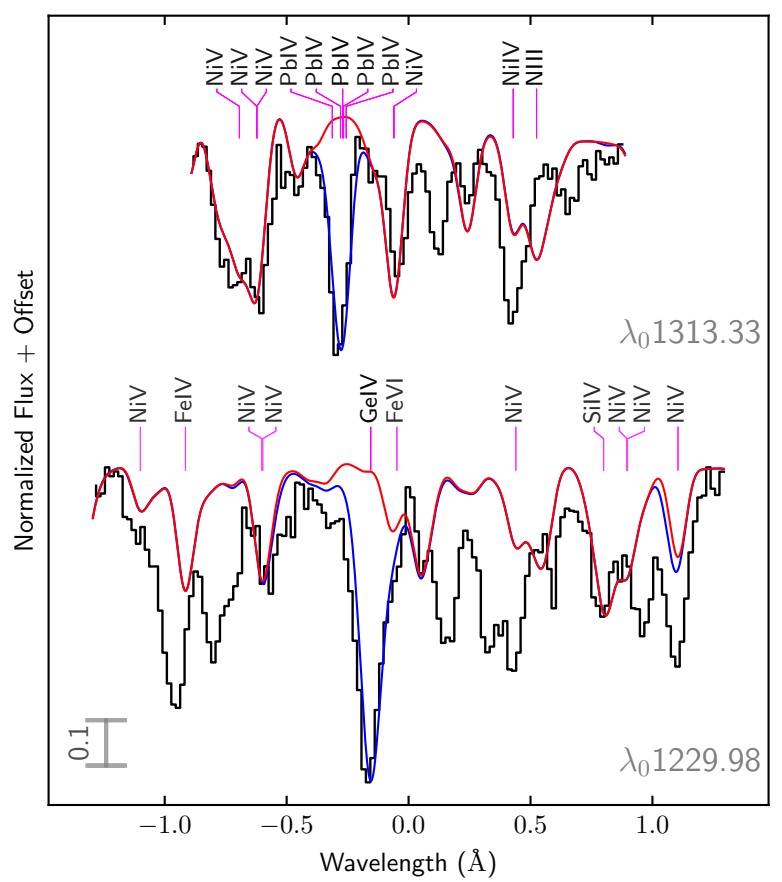

Fig. 7. Ge IV and $\mathrm{Pb}$ IV lines in the GHRS spectrum of HD 127493. In blue: a model with $\log \mathrm{Ge} / \mathrm{H}=-5.0, \log \mathrm{Pb} / \mathrm{H}=-5.7$. In red: the same model without $\mathrm{Pb}$ and $\mathrm{Ge}$.

from Nielsen et al. (2005). Many Ga lines are observed in the UV spectra of HZ 44 and HD 127493. The strongest, isolated lines in HZ 44 include Ga IV $\lambda \lambda 1163.609,1170.585,1258.801$, $1299.476,1303.540$, and $1347.083 \AA$. However, a precise abundance measurement is difficult since most lines are relatively weak and blended with lines from other elements. Nevertheless, we measured an abundance of $\log \mathrm{Ga} / \mathrm{H}=-6.0 \pm 0.5$ (440 times solar) for $\mathrm{HZ} 44$. We only derive an upper $\operatorname{limit}$ of $\log \mathrm{Ga} / \mathrm{H} \leq$ $-6.4 \pm 0.4$ (80 times solar) for HD 127493 since all Ga lines are weaker and blended.

Germanium shows many lines in the FUSE and IUE spectral range, including the strong resonance lines Ge IV $\lambda \lambda 1189.028$, 1229.840 $\AA$, and Ge III 1088.463 $\AA$. We identified lines from Ge III-V in HZ44 that can be matched at an abundance of $\log \mathrm{Ge} / \mathrm{H}=-5.9 \pm 0.3$. (140 times solar). For HD 127493, we derive an abundance of $\log \mathrm{Ge} / \mathrm{H}=-5.0 \pm 0.4$ (470 times solar) from the strong resonance lines Ge IV $1189.028 \AA$ (IUE) and Ge IV $1229.840 \AA$ (GHRS, shown in Fig. 7).

Morton (2000) lists oscillator strengths for ten ultraviolet arsenic III lines from low-lying levels, as computed by Marcinek \& Migdalek (1993). Oscillator strengths for several optical As IV lines are listed in ALL, originally from Churilov \& Joshi (1996). The only ultraviolet As IV line listed in Morton (2000) is the resonance line As IV 1299.28 $\AA$ but the oscillator strength provided by Curtis (1992) is low $\left(f=1.94 \times 10^{-3}\right)$. Atomic data for the two resonance lines As V 987.651 $\AA$ and As V 1029.480 $\AA$ are provided by Pinnington et al. (1981), as listed in Morton (2000). These As V oscillator strengths have previously been used for the As abundance measurement in DO white dwarfs by Chayer et al. (2015) and Rauch et al. (2016a). Morton (2000) also lists a third resonance line, As V 1001.211 A. This line is not observed in the spectrum of HZ 44 and was disregarded by both Chayer et al. (2015) and Rauch et al. (2016a). It is only mentioned in Froese Fischer (1977) (and may have been confused with the ${ }^{2} \mathrm{~S}_{1 / 2}-{ }^{2} \mathrm{P}_{1 / 2}$ transition line As V 1029.480 $\mathrm{A}$ ).

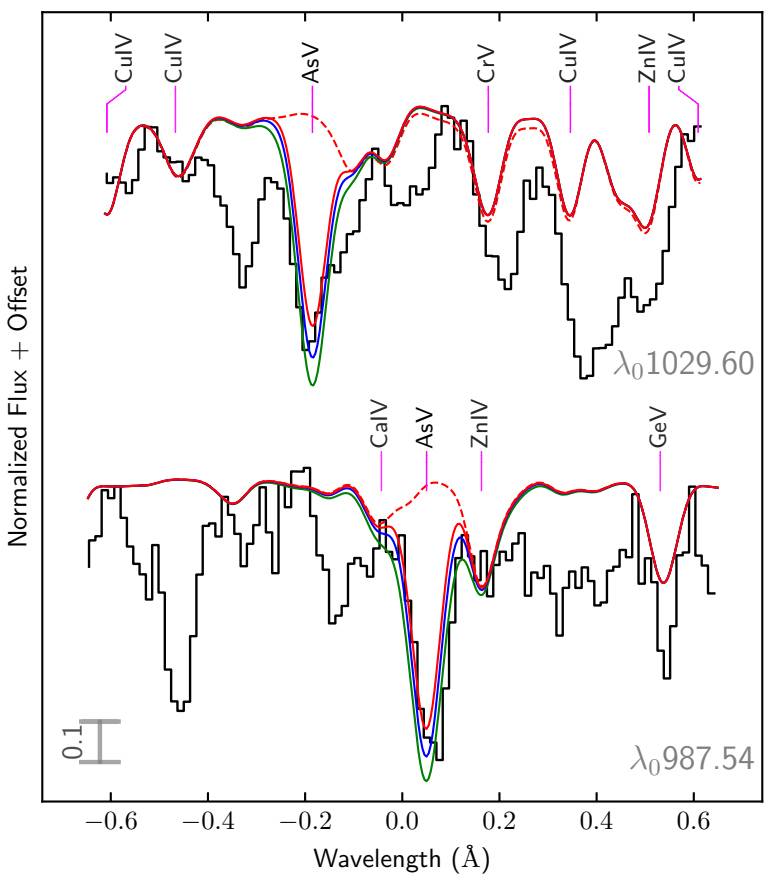

Fig. 8. As v $987.651 \AA$ and As v $1029.480 \AA$ in the FUSE spectrum of HZ 44. In red: a model with $\log \mathrm{As} / \mathrm{H}=-7.8$; dashed without As. In blue: the adopted abundance $\log \mathrm{As} / \mathrm{H}=-7.4$. In green: $\log \mathrm{As} / \mathrm{H}=$ -7.0 .

Neither NIST (Moore 1971) nor Joshi \& van Kleef (1986) list an energy level that would be consistent with an As V resonance line at 1001.211 $\AA$, so we decided to exclude it as well. As III lines are weak in HZ 44 and As III $\lambda \lambda$ 927.540, 944.726 $\AA$ exclude abundances higher than $\log \mathrm{As} / \mathrm{H} \leq-6.4 \pm 0.4$ (960 times solar). As IV $1299.28 \AA$ would fit an otherwise unidentified line at an abundance of $\log \mathrm{As} / \mathrm{H}=-5.6$, which is excluded by other lines. Figure 8 shows the strongest observed As lines in HZ 44, As V $987.651 \AA$, and As V 1029.480 $\mathrm{A}$. We use these lines to derive an abundance of $\log \mathrm{As} / \mathrm{H}=-7.4 \pm 0.4$ (100 times solar). As IV $1299.28 \AA$ is also visible in the GHRS spectrum of HD 127493 and fits the observation at an abundance of $\log \mathrm{As} / \mathrm{H}=-4.9 \pm 0.4$ (1300 times solar). Due to the discrepancy observed in HZ 44, we do not consider this an abundance measurement.

Also as part of their "Stellar Laboratories" series, Rauch et al. (2017b) have measured the abundance of selenium in the peculiar DO white dwarf RE0503-289. We use their oscillator strengths for Se V and results from Bahr et al. (1982) for Se IV, as listed in Morton (2000). Se IV $\lambda \lambda$ 959.590, $996.710 \AA$, and Se V 1094.691 $\AA$ fit strong, otherwise unidentified lines at $\log \mathrm{Se} / \mathrm{H}=-6.3 \pm 0.6$. However, Se IV $984.341 \AA$ seems to exclude abundances higher than $\log \mathrm{Se} / \mathrm{H} \leq-7.6 \pm 0.4$. Like As V $1001.211 \AA$ this line may not be real; its lower level is not listed in NIST nor in the newest reference on Se IV energy levels we found, Pakalka et al. (2018). Therefore, we adopt an abundance of $\log \mathrm{Se} / \mathrm{H}=-6.3 \pm 0.6$ (110 times solar) for $\mathrm{HZ} 44$.

HZ 44 is one of the very few hot subdwarf stars showing zirconium in its optical spectrum. We used the atomic data from Rauch et al. (2017a) for our analysis. We fit four distinct $\mathrm{Zr}$ IV lines in the HIRES spectrum of HZ 44 (Zr IV $\lambda \lambda$ 3576.107, 3686.902, 4317.077, 4569.218, 4569.272 ^, see Fig. 9) and found an abundance of $\log \mathrm{Zr} / \mathrm{H}=-5.92 \pm 0.19$ (1500 times solar). The UV spectra of HZ 44 also show Zr IV-V lines, and although 


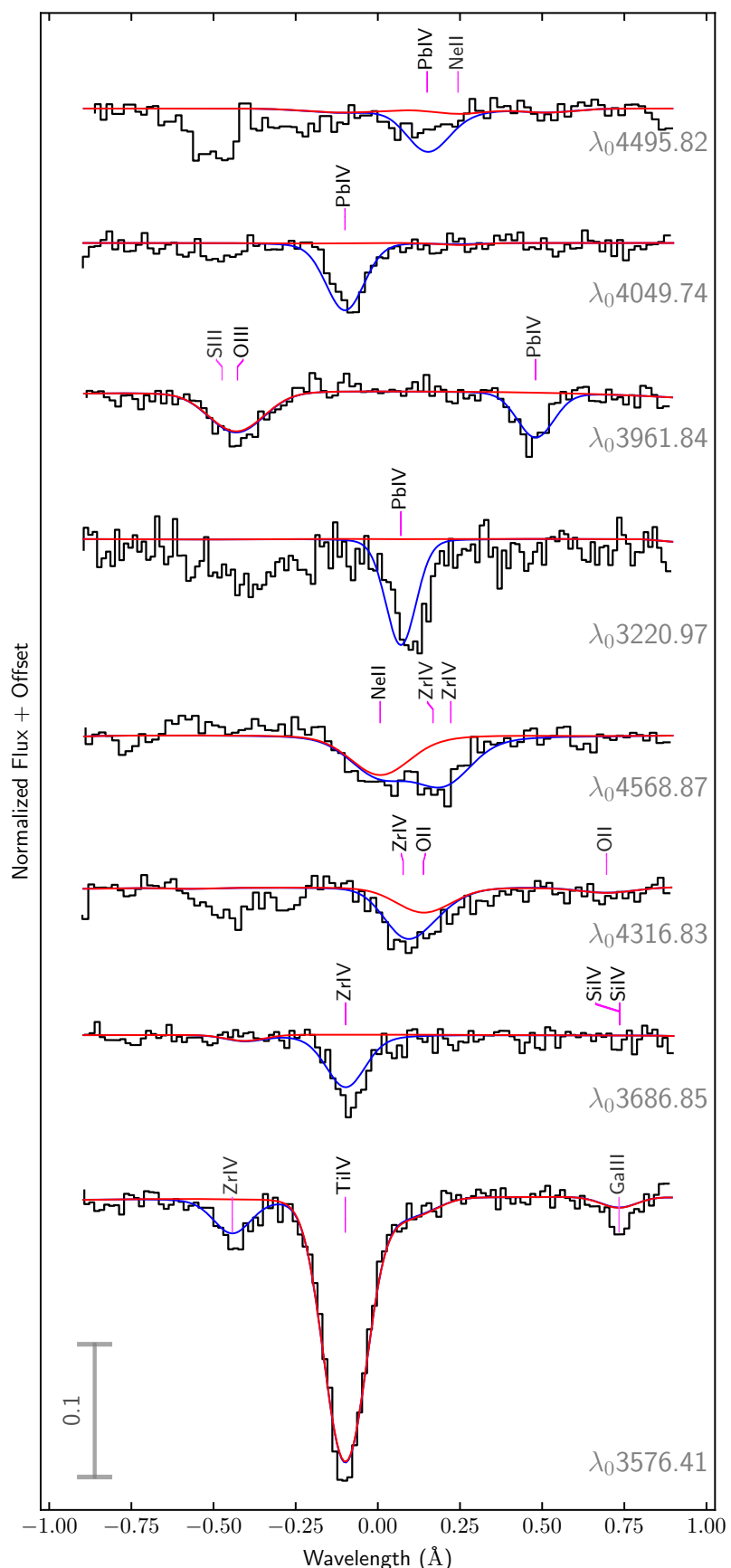

Fig. 9. Selection of $\mathrm{Zr}$ IV and $\mathrm{Pb}$ IV lines in the HIRES spectrum of HZ 44. In blue: a model with $\log \mathrm{Zr} / \mathrm{H}=-5.9$ and $\log \mathrm{Pb} / \mathrm{H}=-5$.9. In red: the same model, but without $\mathrm{Zr}$ or $\mathrm{Pb}$.

none of them are strong or isolated enough to independently measure the abundance, they are consistent with the abundance derived from the optical lines. The doublet $\operatorname{Zr}$ IV $\lambda \lambda 4569.218$, 4569.272 $\AA$ is visible in HD 127493 and would fit well with an abundance of $\log \mathrm{Zr} / \mathrm{H}=-5.5$ (1700 times solar). Since no other lines could clearly be identified as $\mathrm{Zr}$, we adopt an upper limit of $\log \mathrm{Zr} / \mathrm{H} \leq-5.4 \pm 0.2$.

Tin is one of the elements that was identified in HZ 44 by O'Toole (2004). We used atomic data from Safronova et al. (2003), supplemented with data from Biswas et al. (2018) for Sn IV and results from Haris \& Tauheed (2012) for Sn III. We derived the $\mathrm{Sn}$ abundance in $\mathrm{HZ} 44$ to be $\log \mathrm{Sn} / \mathrm{H}=-6.9 \pm 0.4$ (550 times solar), based on the strong Sn IV $1119.338 \AA$ line,

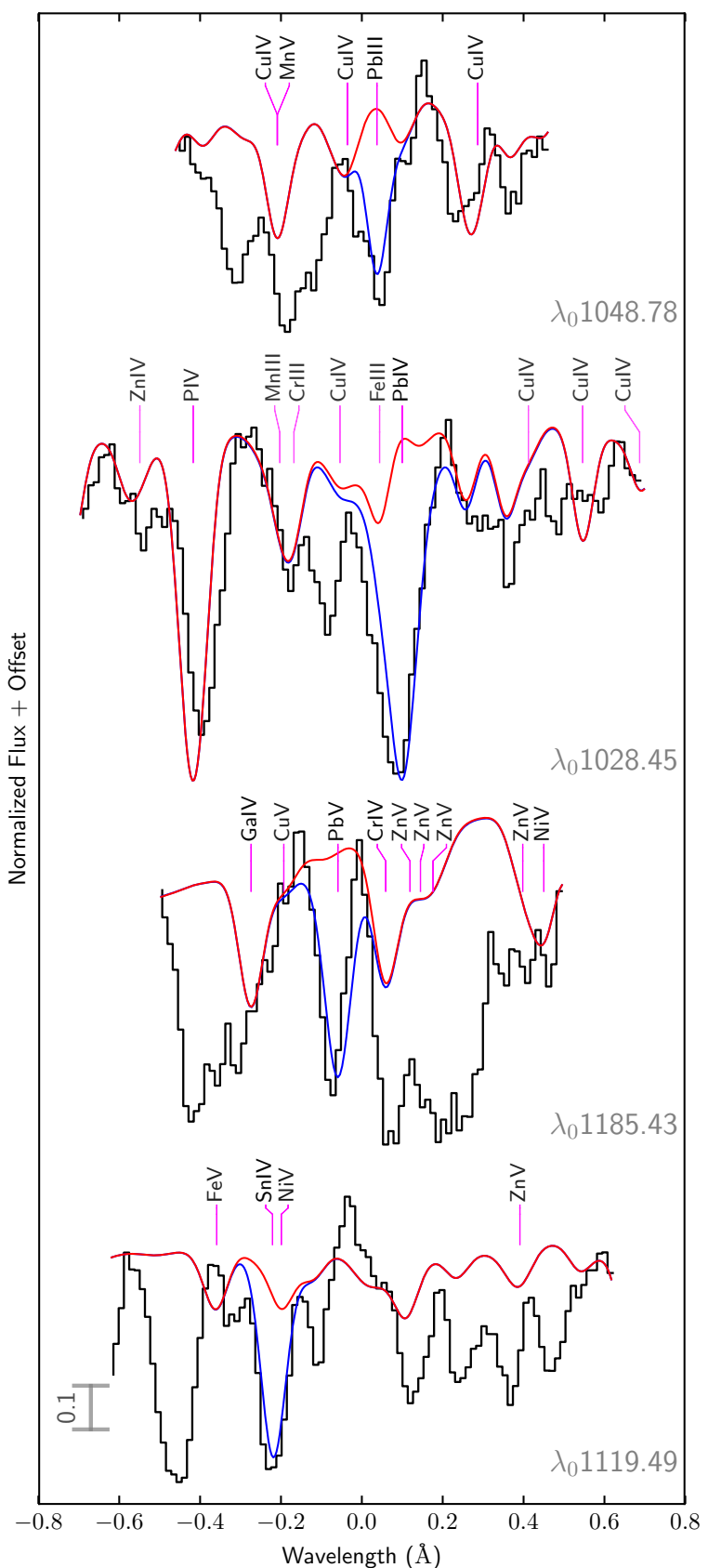

Fig. 10. $\mathrm{Pb}$ III, $\mathrm{Pb} I V, \mathrm{~Pb}$, and $\mathrm{Sn} I V$ lines in the FUSE spectrum of $\mathrm{HZ}$ 44. In blue: a model with $\log \mathrm{Pb} / \mathrm{H}=-5.9$ and $\log \mathrm{Sn} / \mathrm{H}=-6.9$. In red: the same model without $\mathrm{Pb}$ and $\mathrm{Sn}$.

which is almost free from blends (see Fig. 10). The other strong, but blended Sn IV $\lambda \lambda$ 1019.720, 1044.487, 1314.539 , and Sn IV $1437.527 \AA$ (blended with Co IV 1437.488 ̊) lines support this measurement. Even if the FUSE continuum estimation is too high in our models, Sn lines in the IUE spectrum, where the model is more complete, set the upper limit to $\log \mathrm{Sn} / \mathrm{H} \leq-6.5 \pm 0.4$ (1400 times solar). The Sn IV $1314.539 \AA$ line excludes abundances higher than $\log \mathrm{Sn} / \mathrm{H} \leq-6.6 \pm 0.4$ (480 times solar) for HD 127493.

We collected atomic data for lead III-V from several sources. $\mathrm{Pb}$ III oscillator strengths are from Alonso-Medina et al. (2009), with the exception of the resonance lines Pb III 1048.877 $\AA$ and Pb III 1553.021 $\AA$, which are based on lifetime measurements by Ansbacher et al. (1988) as listed in Morton (2000). For Pb IV, 
we use oscillator strengths from Safronova \& Johnson (2004) with additional lines from Alonso-Medina et al. (2011) and one line (Pb IV 4496.15 $\AA$ ) from Naslim et al. (2013). Data for Pb V are provided by Colón et al. (2014). While this collection is far from complete, many $\mathrm{Pb}$ lines could be identified, including not only strong $\mathrm{Pb}$ III-V lines in the ultraviolet spectrum of HZ 44 but also five $\mathrm{Pb}$ IV lines in its optical spectrum ( $\mathrm{Pb}$ IV $\lambda \lambda 3052.56$, 3221.17, 3962.48, 4049.80, 4496.15 A, see Fig. 9).

Fitting all identified $\mathrm{Pb}$ lines in the HIRES spectrum except $\mathrm{Pb}$ IV $3052.56 \AA$ (S/N too low) results in $\log \mathrm{Pb} / \mathrm{H}=$ $-5.89 \pm 0.09$ (11 000 times solar). This is remarkably consistent with $\mathrm{Pb}$ lines observed in the UV region, including lines from $\mathrm{Pb}$ III and $\mathrm{Pb} \mathrm{V}$. As far as we know, this is the first time $\mathrm{Pb}$ V lines were modeled in any star. The strongest $\mathrm{Pb}$ lines per ionization stage observed in the FUSE spectrum of HZ44 are shown in Fig. 10. The $\mathrm{Pb}$ abundance measurement in HD 127493 is based mostly on Pb IV $1313 \AA$ (see Fig. 7), assuming a solar isotopic ratio as in O'Toole \& Heber (2006). We derive an abundance of $\log \mathrm{Pb} / \mathrm{H}=-5.65 \pm 0.40$ (8400 times solar), consistent with $\mathrm{Pb}$ v $\lambda \lambda 1233.50,1248.46 \AA$ in the GHRS spectrum.

\subsection{Trans-iron elements with upper limits}

The abundance measurements for Se, Kr, Sr, Y, Mo, Sb, Te, $\mathrm{Xe}$, and Th turned out to be inconclusive because too few lines were found and/or their relative line strengths were at variance with model predictions. Instead we derived upper limits for these elements.

Krypton and strontium belong to the group of elements that have been studied in white dwarfs by Rauch et al. (2016b, 2017b). Despite the large number of Kr IV-V lines in the TOSS line list, none of them are strong enough in the final synthetic spectrum of HZ 44 to be identified in the observation. We derive an upper limit of $\log \mathrm{Kr} / \mathrm{H} \leq-5.2 \pm 0.6$ (1700 times solar) from four undetected KrIV lines, the strongest being

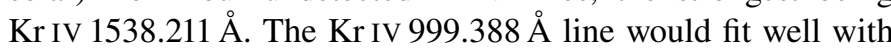
an abundance of $\log \mathrm{Kr} / \mathrm{H}=-5$ but is likely blended. The Kr IV $\lambda \lambda$ 1400.898, 1538.211, $1558.514 \AA$, and Kr V $1293.917 \AA$ lines in the GHRS spectrum of HD 127493 exclude abundances higher than $\log \mathrm{Kr} / \mathrm{H} \leq-4.8 \pm 0.4$ (1900 times solar).

The situation is similar for strontium in HZ 44. The undetected Sr V 962.378 A, Sr IV 1244.137 A, and Sr IV $1244.763 \AA$ lines exclude abundances higher than $\log \mathrm{Sr} / \mathrm{H} \leq-5.1 \pm 0.6$ (5100 times solar). The Sr IV 1331.129 ̊ line would fit well with $\log \mathrm{Sr} / \mathrm{H}=-5.0$. The Sr IV $\lambda \lambda 1244.137,1244.888,1268.622$, 1275.354, 1729.533 $\AA$ lines in GHRS exclude abundances higher than $\log \mathrm{Sr} / \mathrm{H} \leq-4.9 \pm 0.3$ (3600 times solar) in HD 127493 .

Naslim et al. (2011) have observed yttrium in the iHe hot subdwarf LS IV $-14^{\circ} 116$. So far it has been observed to be extremely enriched in two additional iHe-sds: HE 2359-2844 (Naslim et al. 2013) and [CW83] 0825+15 (Jeffery et al. 2017). We used their oscillator strengths for Y III $4039.602 \AA$ and Y III $4040.112 \AA$ to search for $\mathrm{Y}$ in $\mathrm{HZ} 44$ and HD 127493. Both lines are predicted to be weak in the models and were not detected in the spectra of either star. We derive an upper limit of $\log \mathrm{Y} / \mathrm{H} \leq-5.3 \pm 0.2$ (14800 times solar) for HZ 44. Due to the lower S/N of the FEROS spectrum and the higher temperature in HD 127493, the upper limit derived from the same lines in that star is even higher: $\log \mathrm{Y} / \mathrm{H} \leq-4.7 \pm 0.3$ (26000 times solar). None of the ultraviolet Y III lines for which Redfors (1991) computed oscillator strengths are strong enough to improve on this threshold. Unfortunately, we found no oscillator strength measurements for the resonance lines Y III 1000.563 $\AA$ and Y III 1006.587 $\AA$ that are listed in Morton (2000). A more complete analysis of Y in subdwarf stars would benefit from oscillator strengths for Y IV, which is the dominant ionization stage at effective temperatures around $40000 \mathrm{~K}$.

Rauch et al. (2016a) have observed molybdenum in RE 0503-289. We use their atomic data for Mo V and atomic data from the Kurucz line list for Mo IV to search for Mo in HZ 44. The Mo IV $\lambda \lambda$ 965.485, 966.638 $\AA$ and Mo v $\lambda \lambda$ 939.248, $1127.101 \AA$ lines are well reproduced with $\log \mathrm{Mo} / \mathrm{H}=-6.4$ (2500 times solar). Since these lines are relatively weak and our model is missing opacity in the FUSE region, we adopt an upper limit of $\log \mathrm{Mo} / \mathrm{H} \leq-6.2 \pm 0.4$ (4000 times solar). Several lines in the IUE spectrum return a non-detection compatible with this upper limit, for example Mo v $\lambda \lambda$ 1586.898, 1774.317 ̊. As for HD 127493 Mo v $\lambda \lambda$ 1586.898, 1590.414, $1653.5411661 .2151774 .317 \AA$ exclude abundances higher than $\log \mathrm{Mo} / \mathrm{H} \leq-6.1 \pm 0.4$ (2200 times solar).

Werner et al. (2018) have recently measured photospheric abundances of antimony in two DO white dwarfs: RE 0503-289 and PG 0109+111. Both stars are chemically peculiar, with strong enrichment of trans-iron elements. Despite blends with unidentified lines in FUSE and the low resolution of IUE, we were able to set the upper limit on the $\mathrm{Sb}$ abundance in HZ 44 to $\log \mathrm{Sb} / \mathrm{H} \leq-8.0 \pm 0.5$ (470 times solar), which is well below the extreme enrichment observed in the two aforementioned white dwarfs. This upper limit is based on three lines: Sb IV $1042.190 \AA$ (blended with Cr IV), Sb v 1104.23 $\AA$, and Sb v 1226.001 $\AA$. The Sb V $1104.23 \AA$ line even fits well at this abundance.

Zhang et al. (2013) have computed oscillator strengths for tellurium II-III, including UV and optical lines. However, since the Te III population numbers are low in $\mathrm{HZ} 44$, none of these lines are visible. Rauch et al. (2017b) provided oscillator strengths for Te VI lines, including the resonance lines Te VI $\lambda \lambda$ 951.021, $1071.414 \AA$, which are visible in HZ 44 (see Fig. 11). They are best reproduced with an abundance of $\log \mathrm{Te} / \mathrm{H}=-7.5 \pm 0.4$ (100 times solar). Due to the weakness of the lines and their position in the FUSE spectrum we only adopt an upper limit of $\log \mathrm{Te} / \mathrm{H}=-7.1 \pm 0.4$ (250 times solar). The Te V $1281.670 \AA$ resonance line listed in Morton (2000) also supports this upper limit. The Te V $1281.670 \AA$ line is also visible in the GHRS spectrum of HD 127493, but blended with a weaker Co V line. We derive an upper limit of $\log \mathrm{Te} / \mathrm{H}=-5.8 \pm 0.4$ (2200 times solar).

We use oscillator strengths for xenon IV-V from Rauch et al. (2017a), provided on the TOSS website, in order to look for $\mathrm{Xe}$ in $\mathrm{HZ} 44$. Most Xe lines in the FUSE spectrum of HZ 44 are blended with unidentified lines. The resonance lines Xe IV $\lambda \lambda$ 935.251, 1003.373 $\AA$ and Xe V $\lambda \lambda$ 936.284, 945.248 allow us to set an upper limit of $\log \mathrm{Xe} / \mathrm{H} \leq-7.3 \pm 0.4$ (140 times solar). The actual Xe abundance in HZ 44 might be close to our upper limit, since the additional opacity improves the fit for many lines. However, the only isolated line that could be identified is Xe v 936.284 A.

Thorium is the heaviest element for which we found atomic data. It is of particular interest since it is not produced through the s-process and can be used for age determination because of its radioactivity. Safronova \& Safronova (2013) have computed atomic properties of 24 low-lying states of the Th IV ion. Atomic data for Th III are published in Safronova et al. (2014), but could not be used here since all transitions with calculated oscillator strengths lie in the infrared region. Even with a relatively low abundance of $\log \mathrm{Th} / \mathrm{H}=-8.5$ (1400 times solar), our models predict several Th IV lines with estimated equivalent widths 


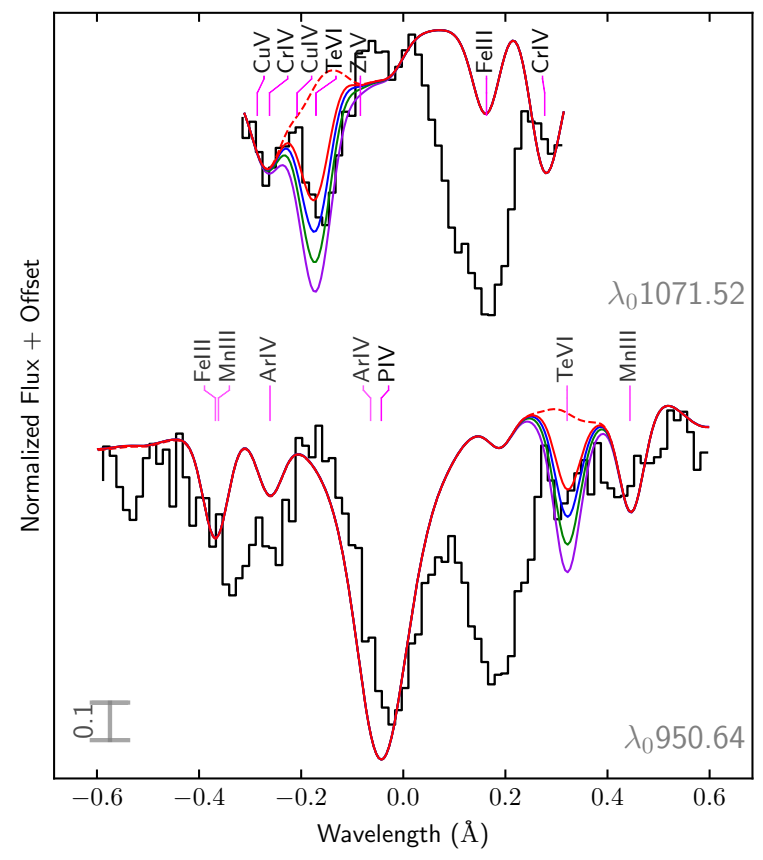

Fig. 11. Te VI resonance lines in the FUSE spectrum of HZ 44. We show a model with $\log \mathrm{Te} / \mathrm{H}=-7.9$ (red) and without $\mathrm{Te}$ (dashed). Models including higher $\mathrm{Te}$ abundances are further shown: $\log \mathrm{Te} / \mathrm{H}=-7.5$ (blue), $\log \mathrm{Te} / \mathrm{H}=-7.1$ (green), and $\log \mathrm{Te} / \mathrm{H}=-6.7$ (pink).

up to $30 \mathrm{~m} \AA$ in the UV range. In particular, the non-detection of Th IV $983.140 \AA$ (which falls conveniently on one of the few points of pseudo-continuum in the FUSE spectrum of HZ 44), Th IV $1140.612 \AA$ (in the wing of a well-modeled Si III line), and Th IV 1682.213 $\AA$ allow us to set the upper limit for the photospheric Th abundance in $\mathrm{HZ} 44$ to $\log \mathrm{Th} / \mathrm{H} \leq-8.0$ (4600 times solar). We derive an upper limit of $\log \mathrm{Th} / \mathrm{H} \leq-7.8 \pm 0.3(3200$ times solar) for HD 127493 based on the Th IV $\lambda \lambda 4413.576$, 5420.380, 5841.397, 6019.151 ̊ lines in the FEROS spectrum.

\subsection{An unsuccessful search for additional trans-iron elements}

We also searched for predicted lines of $\mathrm{Sc}$, In, $\mathrm{Ba}, \mathrm{Tl}$, and $\mathrm{Bi}$ in the ultraviolet and optical spectra of HZ 44. However, no lines from these elements could be identified and no meaningful upper limit could be derived either.

The strongest scandium lines in the model of HZ 44 are the resonance lines Sc III 1610.194 $\AA$ and Sc III 1603.064 $\AA$. Both lines are weak in the model, even at a high abundance of -5 dex relative to hydrogen and are blended with both modeled and unidentified lines, so no meaningful upper limit could be determined.

Safronova et al. (2003) have calculated atomic properties along the silver isoelectronic sequence, including In III, Sn IV, and Sb V. They predict indium III lines from low-lying levels with high oscillator strengths in the IUE spectral region. However, the population numbers for In III are too low to set a meaningful upper limit for both HZ 44 and HD 127493.

Barium was observed in RE 0503-289 by Rauch et al. (2014) who also provide atomic data for $\mathrm{Ba} \mathrm{V}$. The predicted $\mathrm{Ba} \mathrm{V}$ lines are so weak in the model of $\mathrm{HZ} 44$ that $\log \mathrm{Ba} / \mathrm{H}=-5(32000$ times solar) is required before the strongest predicted lines in synthetic spectrum of $\mathrm{HZ} 44$ (Ba V $\lambda \lambda 1097.415,1103.140 \AA$ ) reach equivalent widths of $0.1 \mathrm{~m} \AA$. It was therefore not possible to set a meaningful upper limit.
The strongest observable bismuth line in the spectrum of HZ 44 is by far Biv $1139.549 \AA$. Since it is blended with an unidentified line, we can only derive an upper limit of $\log \mathrm{Bi} / \mathrm{H} \leq$ -8.5 (340 times solar). We consider this upper limit preliminary since it is based on a single, blended line in the FUSE spectrum.

Oscillator strengths along the gold isoelectronic sequence were computed by Safronova \& Johnson (2004), including not only $\mathrm{Pb}$ IV but also thallium III. Similar to In III, the population number for Tl III is too low to set a meaningful upper limit in both HZ 44 and HD 127493.

\subsection{Chemical composition summary}

Figures 12 and 13 as well as Table A.5 show our final abundance values for HZ 44 and HD 127493. The abundance patterns are remarkably similar in both stars despite the $\sim 2500 \mathrm{~K}$ difference in their effective temperature. The overall resemblance between the chemistry of both stars is especially visible when comparing their abundances in number fractions (Fig. 13). In addition, some trans-iron elements are present in the atmosphere with very similar abundances. For example, in $\mathrm{HZ} 44 \mathrm{Cu}, \mathrm{Zn}, \mathrm{Ga}, \mathrm{Ge}, \mathrm{Zr}$, and $\mathrm{Pb}$ have the same number fraction of $\sim 10^{-6.2}$, whereas the solar abundances show a strong decrease with increasing atomic mass. The elements As and Sn are significantly less abundant than the other trans-iron elements.

Both stars show a strong CNO cycle pattern, most obvious in Fig. 13, with nitrogen being enriched while carbon and oxygen are depleted with respect to solar values. $\mathrm{Ne}$ is mildly enriched (by a factor of three) in both stars compared to solar. With the exception of $\mathrm{Cl}$, all elements with $11 \leq Z \leq 20$ are more abundant in HZ 44 than in the Sun. The abundance of $\mathrm{Mg}, \mathrm{Al}, \mathrm{Si}$, and $\mathrm{S}$ is similar in both stars. With a measured abundance of $148_{-61}^{+98}$ times solar, the Ti IV lines are very strong in the UVA spectrum of HZ 44. In contrast, the Ti lines covered by the FEROS spectrum of HD 127493 are weak and set an upper limit for Ti to $94^{+105}$ times solar. Co and $\mathrm{Ni}$ have very similar abundances in both stars: they are about 30 times the solar values. $\mathrm{Mn}$ and $\mathrm{Cu}$ could not be detected in HD 127493, which indicates that they are less abundant than in HZ 44. As seen in many other hot subdwarf stars, $\mathrm{Fe}$ is the least enriched element among the iron group in HZ $44(\sim 1.5$ times solar). Fe is more enriched ( $\sim 10$ times solar) in HD 127493. The $\mathrm{Zn}$ abundance in both stars is similar to that of $\mathrm{Ni}$, between 25 and 30 times solar. While the Ge abundances in HD 127493 ( 470 times solar) and HZ 44 ( 140 times solar) are similar considering uncertainties, the $\mathrm{Ga}$ abundance in HD 127493 ( $\$ 75$ times solar) is lower than in HZ $44(\sim 440$ times solar). The As abundance exceeds that of the Sun by a factor of about 100 in HZ 44. Interestingly, HZ 44 is one of the few hot subdwarf stars showing $\mathrm{ZrIV}$ and Pb IV lines in their optical spectrum. As far as we know, Zr IV has been identified in the optical spectrum of only two iHe hot subdwarfs, LS IV-14 ${ }^{\circ} 116$ and HE 2359-2844 (Naslim et al. 2011, 2013). Zr is enriched at about 1500 times solar in HZ 44, which is not excluded also in HD 127493. In addition, the measured $\mathrm{Pb}$ abundance in both stars is almost identical; they are enhanced by a factor of 10000 . The enrichment is significantly lower for other heavy elements in HZ 44. In particular Xe and Te exceed the solar abundance by a factor of less than 500 in HZ44.

\section{Discussion}

The Carnegie Yearbook No. 55 for $1955 / 56$ reports the discovery of a new sdO star, BD $+25^{\circ} 4655$, to be similar to HD 127493 and HZ 44 and quotes a very foresighted conclusion (probably 


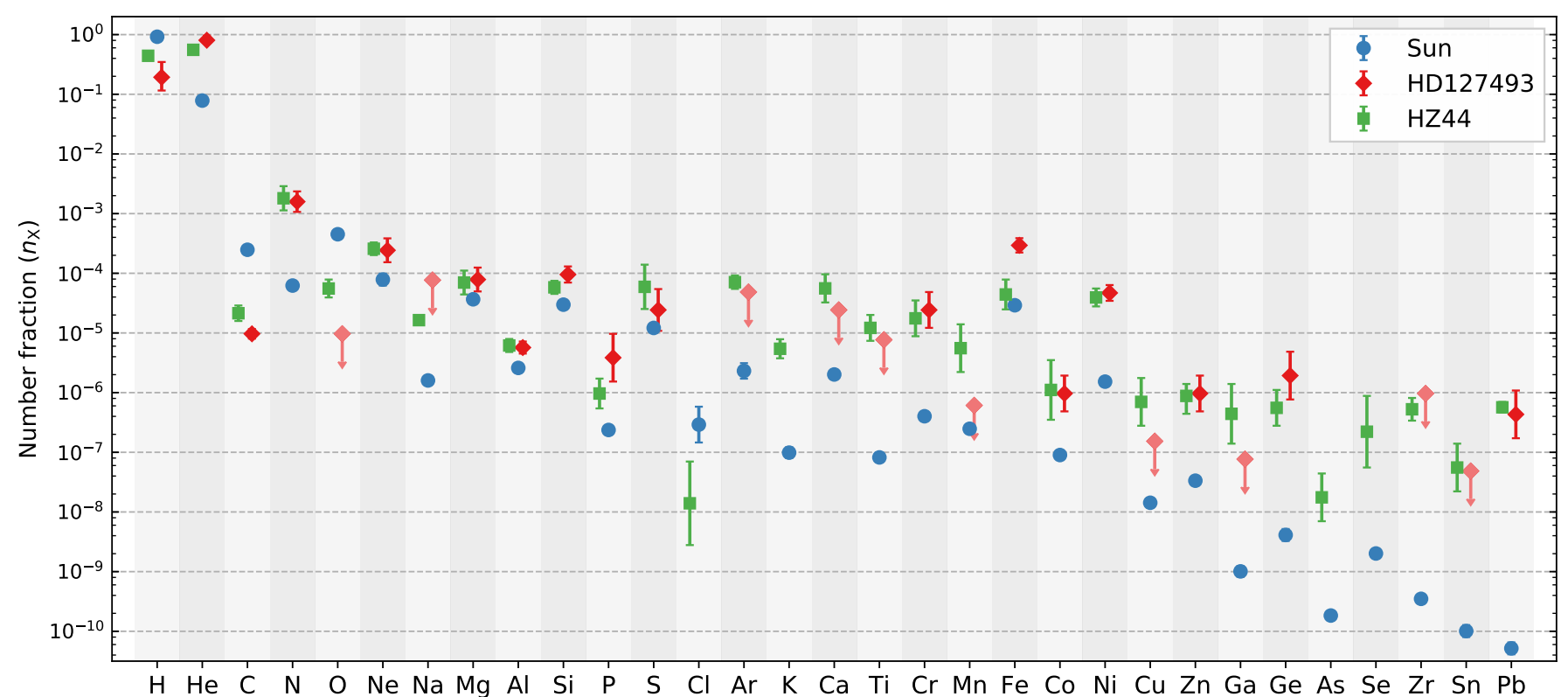

Fig. 12. Abundance patterns of HD 127493 and HZ 44 compared to that of the Sun (by number fraction). Only elements with an abundance measurement in at least one of the stars are shown. Upper limits are marked with an arrow and less saturated colors.

by Münch): that the spectrum of the newly discovered sdO "is extremely rich in faint sharp lines, those of N II, N III, and Ne II being especially conspicuous. The complete absence of lines of oxygen and carbon, in any stage of ionization, suggests that the surface material of this kind of star has undergone nuclear processes which transformed the carbon into nitrogen and the oxygen to neon". The abundance of those elements derived in previous quantitative spectral analyses as well as in ours substantiate this statement. The strong overabundances of heavy elements found for HZ 44, HD 127493, and a few other iHe hot subdwarfs stars are generally believed to be caused by atmospheric diffusion processes (radiative levitation). However, it is not plausible to assume that diffusion creates an abundance pattern of $\mathrm{C}, \mathrm{N}, \mathrm{O}$, and $\mathrm{Ne}$ that mimics the nucleosynthesis pattern so well. In the following subsections, we first discuss the evidence for diffusion processes and compare the abundance patterns of our two stars to that of other iHe hot subdwarfs. Then we revisit the nuclear synthesis aspect and discuss implications for the evolutionary status.

\subsection{Diffusion}

Diffusion refers to the equilibrium between gravitational settling and radiative levitation. While heavy elements are pulled downwards by gravity, their important line opacities in the UV region, where the photospheric flux distribution peaks, lead to opposing forces due to radiation pressure. This force is limited by the saturation of spectral lines at high abundances. Once an equilibrium of both forces has been established, the elemental abundances should be fixed.

Models that account for gravitational settling and radiative levitation, only, fail to reproduce the observed abundance pattern of sdB stars (see Heber 2016, for a discussion). Additional processes have to be taken into account. Stellar winds and turbulent mixing have been suggested. Michaud et al. (2011) have studied the effects of non-equilibrium diffusion and radiative levitation on element abundances up to $\mathrm{Ni}$ for sdB stars on the horizontal branch (up to $T_{\text {eff }} \approx 37000 \mathrm{~K}$ ), but not for sdOs. To match the iron abundances observed in sdBs by Geier et al. (2010), and later Geier (2013), they required some process to dampen the effect of radiative levitation. Michaud et al. (2011) adopted a turbulent surface mixing zone during the horizontal branch (HB) evolution that includes the outer $\sim 10^{-7.5} M_{\odot}$ in the envelope. Similarly to the sdOs discussed in this paper, the photospheric iron abundance in sdBs is approximately solar. This low Fe enhancement is a result of its high absolute abundance in the photosphere and the consequent line saturation (see Fig. 12). Since heavy elements, such as $\mathrm{Zr}$ and $\mathrm{Pb}$, are initially less abundant in absolute terms, a stronger enrichment due to radiative levitation is expected. The models for the hottest stars $\left(T_{\text {eff }}=35-37 \mathrm{kK}\right)$ in Michaud et al. (2011) predict abundances that are lower than what is observed in HZ 44 and HD 127493. For example, N, Ne, $\mathrm{Al}, \mathrm{Si}$, and $\mathrm{Mg}$ are predicted to be depleted with respect to the solar values. Thus additional processes are required to explain the abundance pattern of our two sdOs; nucleosynthesis during the formation of the stars, weak stellar winds (Unglaub 2008; Hu et al. 2011), and a possible atmospheric surface convection zone (Groth et al. 1985; Unglaub 2010) might well be involved.

The models by Michaud et al. (2011) cannot reproduce the He-enrichment and CNO-cycle pattern observed in some sdBs (and the sdOs discussed here) since they use approximated methods to evolve their models through the He-flash. Byrne et al. (2018) have preformed similar calculations for post common envelope sdBs from the top of the RGB to the zero age HB with a more self-consistent treatment of the He-flash. They produced He-rich atmospheres in their delayed He-flash models and predict $\mathrm{C}$ and $\mathrm{N}$ to be enriched and $\mathrm{O}$ to be depleted for sdBs on the zero-age horizontal branch (ZAHB). The abundances of other elements are similar to those of Michaud et al. (2011) but both models are not especially well suited for the hotter stars discussed here. Detailed sdO evolutionary models (e.g., through the HeWD-merger channel) including diffusion of heavy elements beyond the iron group would be required to explain the observed abundance pattern. Unfortunately, the atomic data required for modeling diffusion of elements heavier than $\mathrm{Ni}$ is still lacking.

\subsection{Comparison with other iHe hot subdwarfs}

In Fig. 13 we compared the abundance pattern of HZ 44 and HD 127493 with literature abundances of two other iHe subdwarf 


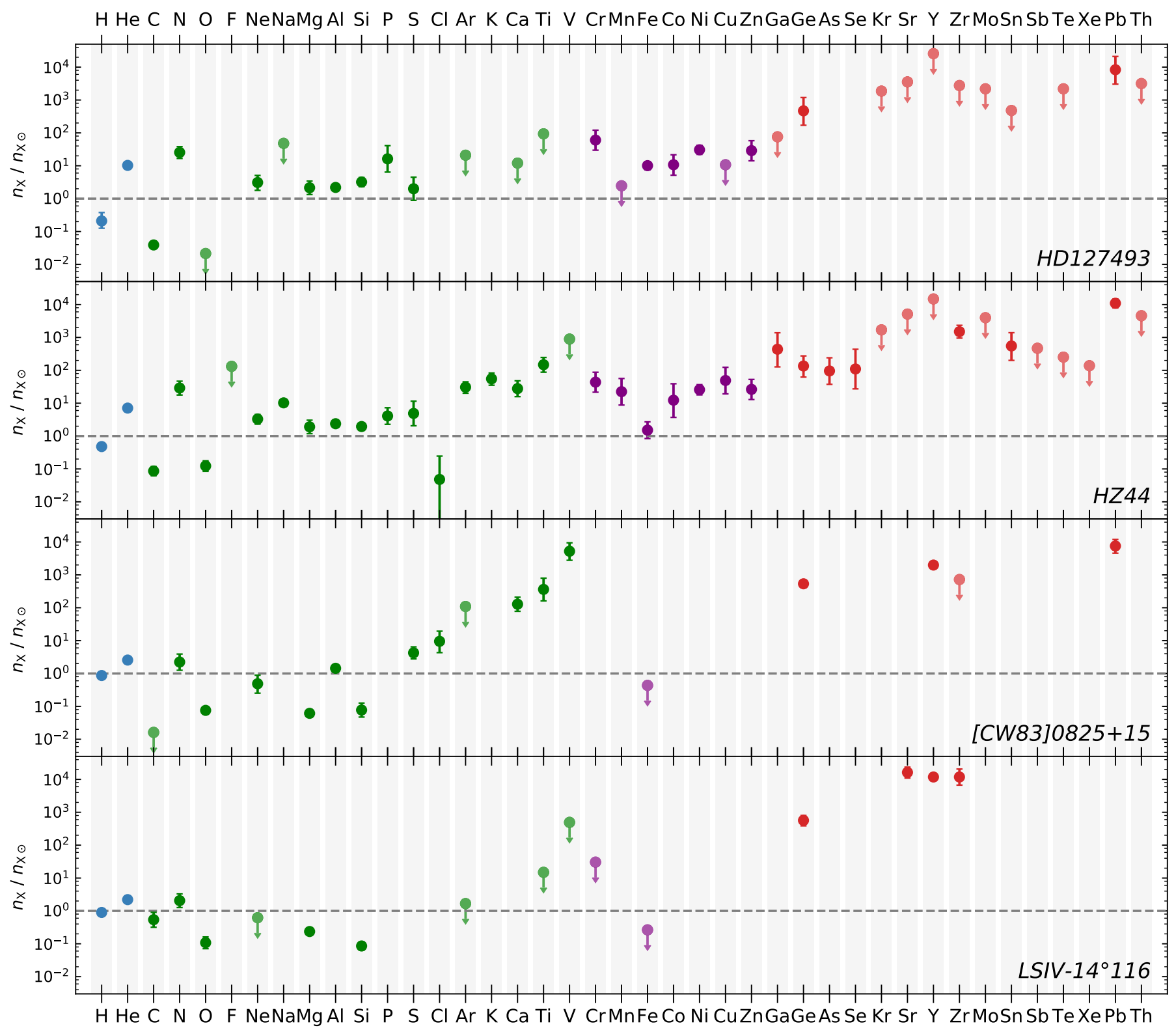

Fig. 13. Abundance pattern of HD 127493 and HZ 44 with respect to solar composition. Results for the heavy-metal subdwarfs [CW83] 0825+15 (Jeffery et al. 2017) and LS IV $-14^{\circ} 116$ (Naslim et al. 2011) are shown for comparison. Light elements $(23 \leq Z)$ are marked by green symbols, iron-peak elements $(24 \leq Z \leq 30)$ in purple, and heavier elements $(Z \geq 31)$ in red. Upper limits are marked with an arrow and less saturated colors.

stars: [CW83] $0825+15$ and LS IV $-14^{\circ} 116$. [CW83] $0825+15$ is the closest match to HZ 44 and HD 127493 in terms of of atmospheric properties with $T_{\text {eff }}=38900 \mathrm{~K}$ and $\log g=5.97$ (Jeffery et al. 2017). Although less He-rich $\left(\log n_{\mathrm{He}} / n_{\mathrm{H}}=-0.6\right)$, it is the only known heavy-metal iHe hot subdwarf to be C-deficient, like the two stars we analyzed here. Its abundance pattern is also similar to HZ 44 and HD 127493 in that the CNO-cycle pattern is evident and lead is equally enriched. However, the abundances of some specific elements differ significantly: $\mathrm{Mg}$ and $\mathrm{Si}$ are less abundant by $\sim 1$ dex while $\mathrm{Cl}$ is about 2 dex more abundant in [CW83] 0825+15.

LS IV $-14^{\circ} 116$ was the first heavy-metal hot subdwarf to be recognized as such and is considered as the prototype of the class, with its extreme enrichment in $\mathrm{Sr}, \mathrm{Y}$, and $\mathrm{Zr}$ (Naslim et al. 2011). With $T_{\text {eff }}=34950 \mathrm{~K}, \log g=5.93$, and $\log n_{\mathrm{He}} / n_{\mathrm{H}}=$ -0.62 (Green et al. 2011), the star is cooler and less helium rich than HZ44 and HD 127493. Similarly to the two other heavy-metal subdwarfs, HE 2359-2844 and HE 1256-2738, its $\mathrm{C}$ abundance is higher than in HZ 44 and HD 127493 (see also Fig. 1). Its $\mathrm{Sr}$ and $\mathrm{Zr}$ enrichment is stronger than in $\mathrm{HZ} 44$ and HD 127493 (and [CW83] 0825+15).

Although the abundances of HZ44 and HD 127493 are remarkably similar, the patterns observed in other heavy-metal subdwarfs appear to be different. However, it is difficult to draw firm conclusions when abundances are known only for a much more limited subset of elements in the other stars. In the case of LS IV $-14^{\circ} 116$ and [CW83] 0825+15, the lack of UV data strongly restricts their chemical portrait. Along with [CW83] 0825+15, our two stars HZ 44 and HD 127493 are the only known heavy-metal subwarfs to be enriched in nitrogen, but depleted in carbon and oxygen. The three other known heavymetal subdwarfs have higher $\mathrm{C}$-abundances, similarly to the group of $\mathrm{CN}$-rich eHe subdwarfs that is observed at higher temperatures. Whether the differences in the abundances of carbon 
and nitrogen in iHe subdwarfs are related to stellar evolution or the effects of diffusion remains unclear.

\subsection{Nuclear synthesis and evolutionary status}

The formation of hot subdwarfs with intermediate He abundances (10-90\% by number) through merging He-WDs with low-mass MS stars was investigated by Zhang et al. (2017). In these models, subdwarfs with intermediate He-rich atmospheres represent a short ( $<5 \mathrm{Myr}$ ) phase after the He-flash is ignited during the merger. The initially He-rich atmosphere of the merger remnant transforms into a $\mathrm{H}$-rich one as the heavier He diffuses downward (gravitational settling) until the atmosphere is H-rich when the ZAHB is reached. The same process is predicted in accretion-based HeWD+HeWD mergers (Zhang \& Jeffery 2012) that can also reproduce the $\mathrm{He}$ abundance in iHe-sds.

A known problem is that merger calculations predict a fast surface rotation. Schwab (2018) has calculated postHeWD+HeWD merger models with initial conditions taken from hydrodynamic merger calculations and found that merger products have $v_{\text {rot }} \geq 30 \mathrm{~km} \mathrm{~s}^{-1}$ once they appear as hot subdwarfs. This rotation is usually not observed in single sdBs (Geier \& Heber 2012) and Hirsch (2009) found N-rich He-sdOs (such as HZ44 and HD 127493) to have $v_{\text {rot }}$ similar to sdBs. For individual stars, this can be explained by a small inclination $i$ (which leads to a small $v_{\text {rot }} \sin i$ ). However, with increasing evidence for slowly rotating (intermediate) He-sdOs, it seems likely that additional physics is needed to match the observations (Schwab 2018). Alternatively, slowly rotating hot subdwarfs may be created through a different process altogether.

The observation of the CNO cycle pattern in HD 127493 and HZ 44 indicates that the $\mathrm{CNO}$ process must have been efficient in a H-burning shell or mixed from a sufficiently hot core in the stars' progenitor. In fact, the slow HeWD+HeWD merger model by Zhang \& Jeffery (2012) is able to reproduce the CNO pattern observed in HZ 44 and HD 127493 well except for somewhat higher predicted $\mathrm{O}$ abundances. This may be an indication that $\mathrm{O}$ has been processed to Ne through the $\alpha$ capture ${ }_{8}^{16} \mathrm{O}(\alpha, \gamma){ }_{10}^{20} \mathrm{Ne}$. In HeWD+MS merger models presented by Zhang et al. (2017), temperatures high enough for ${ }_{8}^{18} \mathrm{O}(\alpha, \gamma){ }_{10}^{22} \mathrm{Ne}$ burning are reached following the first He-flash, even if the processed material is not always mixed to the surface. That the $\mathrm{He}, \mathrm{C}, \mathrm{N}, \mathrm{O}$, and $\mathrm{Ne}$ abundances in some He-sdOs, and in the two stars analyzed here, can be explained by nuclear synthesis might indicate that these light elements are less affected by diffusion in this type of stars.

An alternative explanation to diffusion for the extreme enrichment of heavy element could be that they were created in the stars' progenitor. Heavy elements like $\mathrm{Zr}$ and $\mathrm{Pb}$ are produced mainly in the s-process, which is thought to be efficient in AGB stars. While most hot subdwarfs do not evolve through the AGB phase, low-mass post-AGB tracks cross the $\log g-T_{\text {eff }}$ diagram in the region populated by luminous hot subdwarfs (Napiwotzki 2008). Therefore such an evolutionary channel might be responsible for a small fraction of the hot subdwarfs. However, diffusion calculations for these elements are required before conclusions on possible AGB progenitors of heavy-metal enriched iHe-sds can be made.

\section{Conclusion}

We have performed a detailed spectroscopic analysis of the two intermediate He-sdOs HZ44 and HD 127493. SED fits combined with parallax distances for both stars result in masses that are consistent with the canonical subdwarf mass of $0.47 M_{\odot}$ within $1 \sigma$ uncertainty. No indication of binarity was found for either star. Our main focus was the determination of photospheric metal abundances, including heavy elements. We found the abundance pattern in both stars to be very similar. They show a typical CNO-cycle pattern and slight enrichment of intermediate-mass elements $(Z \leq 30$, except $\mathrm{Cl})$ compared to solar values. Heavier elements such as $\mathrm{Ga}, \mathrm{Ge}$, and As were found to be enriched on the order of 100 times solar. Most interestingly, the abundances of $\mathrm{Zr}$ and $\mathrm{Pb}$ were measured from optical lines and confirmed with UV transitions in HZ44, and turned out to be more than 1000 and 10000 times solar, respectively. HD 127493 shows no optical $\mathrm{Zr}$ or $\mathrm{Pb}$ lines, but we derived a $\mathrm{Pb}$ enrichment of about 8000 times solar from $\mathrm{Pb}$ IV-V lines in its HST/GHRS UV spectrum. Pb V lines were modeled for the first time in a stellar photosphere and their predicted strength reproduced well the observations of both stars. We also determined upper limits for several additional heavy elements. Some of them, for example $\mathrm{Xe}$ and $\mathrm{Te}$, have a moderate enrichment ( $\$ 500$ times solar) in HZ 44.

In order to improve the accuracy of abundance measurements, additional atomic data are much-needed, in particular for the heavy elements. Many lines in both the optical and ultraviolet spectra still remain unidentified. This is especially evident in the FUSE spectrum of HZ 44, where not only interstellar but also many photospheric lines are missing from our models. Some of those lines likely belong to ionized heavy elements for which no atomic data, or only a limited subset, are available.

Interestingly, pulsations were observed in three other iHe subdwarfs, namely [CW83] 0825+15 (Jeffery et al. 2017), LS IV-14 116 (Ahmad \& Jeffery 2005), and Feige 46 (Latour et al. 2019b). This would make it worth looking for photometric variability in HZ 44 and HD 127493 as well.

As of now, we are not able to fully explain the observed abundance pattern in intermediate He-sdOs. Evolutionary simulations for sdOs including diffusion for heavy elements and mixing during hot flasher or merger evolution would be required to interpret the abundance pattern. Even though we obtained a quite exhaustive chemical portrait for the two stars analyzed here, this is generally not the case for the other iHe hot subdwarfs. More complete sets of abundances for additional stars are also necessary to properly investigate these intriguing patterns.

The efficiency of radiative support on heavy elements in hot subdwarfs might be linked to their helium abundance, given that the intermediate helium-rich hot subdwarfs seem to favorably display extreme enhancements. Hydrogen-rich sdB stars were found to be enriched in some heavy elements as well (O'Toole \& Heber 2006; Blanchette et al. 2008), but their enrichment in $\mathrm{Pb}$ for example is significantly lower than that observed in the heavy-metal iHe subdwarfs. At the other end of the helium abundance spectrum, abundance analyses of He-sdOs are more limited, especially concerning heavy metals. The only He-rich $\mathrm{sdO}$ for which heavy-metal abundances have been derived, $\mathrm{BD}+393226$, turned out to be less than 2 dex enhanced in $\mathrm{Zr}$ and $\mathrm{Pb}$ (Chayer et al. 2014). It would be most interesting to determine abundances of heavy elements in additional He-rich stars. The He-sdOs recently analyzed by Schindewolf et al. (2018) would be well-suited to confirm (or not) this milder enrichment in heavy metals. Their atmospheric parameters, as well as their abundances of lighter elements, are well constrained, and excellent UV data are available. The current set of hot subdwarfs for which abundances of heavy elements are known do not allow us to rule out the possibility that the effective temperature also plays a role in favoring the radiative support of particular elements. Once again abundances for a larger sample of stars across 
the $T_{\text {eff }}$ range where the extreme overabundances are observed ( 34-43 kK), also including hydrogen-rich stars such as the two hottest objects from O'Toole \& Heber (2006), will be necessary in order to investigate the relation between $T_{\text {eff }}$ and the (over)abundances of particular elements.

Acknowledgements. We thank Andreas Irrgang and Simon Kreuzer for the development of the SED fitting tool, Monika Schork for measuring the radial velocitie of HZ 44 from HIRES spectra, and Markus Schindewolf for providing preliminary atmospheric parameters of HZ 44. M.L. acknowledges funding from the Deutsche Forschungsgemeinschaft (grant DR 281/35-1). Based on observations made with the NASA/ESA Hubble Space Telescope, obtained from the data archive (prop. ID GO5305) at the Space Telescope Science Institute. STScI is operated by the Association of Universities for Research in Astronomy, Inc. under NASA contract NAS 5-26555. Support for MAST for non-HST data is provided by the NASA Office of Space Science via grant NNX09AF08G and by other grants and contracts. Based on INES data from the IUE satellite. Based on observations made with ESO Telescopes at the La Silla Paranal Observatory under programme ID 074.B-0455(A). This research has made use of the Keck Observatory Archive (KOA), which is operated by the W.M Keck Observatory and the NASA Exoplanet Science Institute (NExScI), under contract with the National Aeronautics and Space Administration. This work has made use of data from the European Space Agency (ESA) mission Gaia (https: //www. cosmos.esa.int/gaia), processed by the Gaia Data Processing and Analysis Consortium (DPAC, https://www.cosmos.esa.int/web/ gaia/dpac/consortium). Funding for the DPAC has been provided by national institutions, in particular the institutions participating in the Gaia Multilateral Agreement. The TOSS service (http://dc.g-vo.org/TOSS) used for this paper was constructed as part of the activities of the German Astrophysical Virtual Observatory. We acknowledge the use of the Atomic Line List (http://www.pa.uky.edu/ peter/newpage/). We also thank the referee, C. Moni Bidin, for his helpful comments.

\section{References}

Ahmad, A., \& Jeffery, C. S. 2005, A\&A, 437, L51

Allende Prieto, C., Lambert, D. L., Hubeny, I., \& Lanz, T. 2003, ApJS, 147, 363 Alonso-Medina, A., Colón, C., \& Zanón, A. 2009, MNRAS, 395, 567

Alonso-Medina, A., Colón, C., \& Porcher, P. 2011, At. Data Nucl. Data Tables, 97,36

Andersen, T., \& Lindgard, A. 1977, J. Phys. B At. Mol. Phys., 10, 2359

Andersen, T., Eriksen, P., Poulsen, O., \& Ramanujam, P. S. 1979, Phys. Rev. A, 20, 2621

Ansbacher, W., Pinnington, E. H., \& Kernahan, J. A. 1988, Can. J. Phys., 66, 402

Asplund, M., Grevesse, N., Sauval, A. J., \& Scott, P. 2009, ARA\&A, 47, 481

Bahr, J. L., Pinnington, E. H., Kernahan, J. A., \& O’Neill, J. A. 1982, Can. J. Phys., 60, 1108

Bauer, F., \& Husfeld, D. 1995, A\&A, 300, 481

Bessell, M. S. 1999, PASP, 111, 1426

Biswas, S., Das, A., Bhowmik, A., \& Majumder, S. 2018, MNRAS, 477, 5605

Blanchette, J.-P., Chayer, P., Wesemael, F., et al. 2008, ApJ, 678, 1329

Bohlin, R. C. 1996, AJ, 111, 1743

Bohlin, R. C., Harris, A. W., Holm, A. V., \& Gry, C. 1990, ApJS, 73, 413

Bohlin, R. C., Dickinson, M. E., \& Calzetti, D. 2001, AJ, 122, 2118

Byrne, C. M., Jeffery, C. S., Tout, C. A., \& Hu, H. 2018, MNRAS, 475, 4728

Chayer, P., Fontaine, M., Fontaine, G., Wesemael, F., \& Dupuis, J. 2006, Balt. Astron., 15, 131

Chayer, P., Green, E. M., \& Fontaine, G. 2014, Amer. Astron. Soc. Meet. Abstr., 223, 154.21

Chayer, P., Dupuis, J., \& Kruk, J. W. 2015, in 19th European Workshop on White Dwarfs, eds. P. Dufour, P. Bergeron, \& G. Fontaine, ASP Conf. Ser., 493, 3

Churilov, S. S., \& Joshi, Y. N. 1996, J. Opt. Soc. Am. B Opt. Phys., 13, 11

Colón, C., Alonso-Medina, A., \& Porcher, P. 2014, At. Data Nucl. Data Tables, 100,272

Curtis, L. J. 1992, J. Opt. Soc. Am. B Opt. Phys., 9, 5

Cutri, R. M., Skrutskie, M. F., van Dyk, S., et al. 2003, VizieR Online Data Catalog: II/246

Cutri, R. M., Wright, E. L., Conrow, T. R. M. \& et al. 2012, VizieR Online Data Catalog: II/311

Dorman, B., Rood, R. T., \& O’Connell, R. W. 1993, ApJ, 419, 596

Dorsch, M., Latour, M., \& Heber, U. 2018, Open Astron., 27, 19

Fitzpatrick, E. L. 1999, PASP, 111, 63

Fontaine, G., Brassard, P., Charpinet, S., et al. 2012, A\&A, 539, A12

Froese Fischer, C. 1977, J. Phys. B: At. Mol. Phys., 10, 1241

Gaia Collaboration 2018, VizieR Online Data Catalog: I/345
Geier, S. 2013, A\&A, 549, A110

Geier, S., \& Heber, U. 2012, A\&A, 543, A149

Geier, S., Heber, U., Edelmann, H., Morales-Rueda, L., \& Napiwotzki, R. 2010, Ap\&SS, 329, 127

Green, E. M., Guvenen, B., O’Malley, C. J., et al. 2011, ApJ, 734, 59

Groth, H. G., Kudritzki, R. P., \& Heber, U. 1985, A\&A, 152, 107

Gruschinske, J., Hunger, K., Kudritzki, R. P., \& Simon, K. 1980, in Ultraviolet observations of Quasars, eds. B. Battrick \& J. Mort, ESA SP, 157, 311

Han, Z., Podsiadlowski, P., Maxted, P. F. L., Marsh, T. R., \& Ivanova, N. 2002, MNRAS, 336, 449

Haris, K., \& Tauheed, A. 2012, Phys. Scr, 85, 055301

Heber, U. 2016, PASP, 128, 082001

Heber, U., Irrgang, A., \& Schaffenroth, J. 2018, Open Astron., 27, 35

Hirsch, H. A. 2009, PhD Thesis, Friedrich-Alexander University ErlangenNürnberg, Germany

Hu, H., Tout, C. A., Glebbeek, E., \& Dupret, M. A. 2011, MNRAS, 418, 195

Hubeny, I. 1988, Comput. Phys. Commun., 52, 103

Hubeny, I., \& Lanz, T. 2017a, ArXiv e-prints [arXiv:1706 . 01859]

Hubeny, I., \& Lanz, T. 2017b, ArXiv e-prints [arXiv:1706.01935]

Hubeny, I., \& Lanz, T. 2017c, ArXiv e-prints [arXiv:1706.01937]

Humason, M. L., \& Zwicky, F. 1947, ApJ, 105, 85

Jeffery, C. S., Baran, A. S., Behara, N. T., et al. 2017, MNRAS, 465, 3101

Joshi, Y. N., \& van Kleef, T. A. M. 1986, Can. J. Phys., 64, 330

Kaufer, A., Stahl, O., Tubbesing, S., et al. 1999, The Messenger, 95, 8

Kilkenny, D., van Wyk, F., Roberts, G., Marang, F., \& Cooper, D. 1998 MNRAS, 294, 93

Kudritzki, R. P. 1976, A\&A, 52, 11

Kurucz, R. L. 2018, ASP Conf. Ser., 515, 47

Landolt, A. U., \& Uomoto, A. K. 2007, AJ, 133, 768

Lanz, T., \& Hubeny, I. 2003, ApJS, 146, 417

Lanz, T., \& Hubeny, I. 2007, ApJS, 169, 83

Lanz, T., Brown, T. M., Sweigart, A. V., Hubeny, I., \& Landsman, W. B. 2004, ApJ, 602, 342

Latour, M., Fontaine, G., Chayer, P., \& Brassard, P. 2013, ApJ, 773, 84

Latour, M., Dorsch, M., \& Heber, U. 2019a, A\&A, in press, https://doi.org/10.1051/0004-6361/201936247

Latour, M., Green, E. M., \& Fontaine, G. 2019b, A\&A, 623, L12

Lawrence, A., Warren, S. J., Almaini, O., et al. 2013, VizieR Online Data Catalog: II/319

Lisker, T., Heber, U., Napiwotzki, R., et al. 2005, A\&A, 430, 223

Marcinek, R., \& Migdalek, J. 1993, J. Phys. B At. Mol. Phys., 26, 1403

Marinoni, S., Pancino, E., Altavilla, G., et al. 2016, MNRAS, 462, 3616

Mashonkina, L., Gehren, T., Travaglio, C., \& Borkova, T. 2003, A\&A, 397, 275

Massey, P., Strobel, K., Barnes, J. V., \& Anderson, E. 1988, ApJ, 328, 315

Menzies, J. W., Marang, F., \& Westerhuys, J. E. 1990, SAAO Circ., 14, 33

Mermilliod, J. C. 2006, VizieR Online Data Catalog: II/168

Michaud, G., Richer, J., \& Richard, O. 2011, A\&A, 529, A60

Migdałek, J. 1983, J. Quant. Spectr. Rad. Transf., 30, 169

Miller Bertolami, M. M., Althaus, L. G., Unglaub, K., \& Weiss, A. 2008, A\&A, 491, 253

Moore, C. E. 1971, in National Standard Reference Data Series, NSRDS-NBS 35, Vol. II (Reprint of NBS Circ. 467, Vol. II, 1952) (U.S.: National Bureau of Standards)

Morton, D. C. 2000, ApJS, 130, 403

Münch, G. 1958, ApJ, 127, 642

Napiwotzki, R. 2008, in Hot Subdwarf Stars and Related Objects, eds. U. Heber, C. S. Jeffery, \& R. Napiwotzki, ASP Conf. Ser., 392, 139

Napiwotzki, R., Karl, C. A., Lisker, T., et al. 2019, A\&A, accepted [arXiv:1906.10977]

Naslim, N., Jeffery, C. S., Behara, N. T., \& Hibbert, A. 2011, MNRAS, 412, 363 Naslim, N., Jeffery, C. S., Hibbert, A., \& Behara, N. T. 2013, MNRAS, 434, 1920

Nielsen, K. E., Wahlgren, G. M., Proffitt, C. R., Leckrone, D. S., \& Adelman, S. J. 2005, AJ, 130, 2312

Oke, J. B. 1990, AJ, 99, 162

O'Reilly, F., \& Dunne, P. 1998, J. Phys. B At. Mol. Phys., 31, 1059

O'Toole, S. J. 2004, A\&A, 423, L25

O’Toole, S. J., \& Heber, U. 2006, A\&A, 452, 579

Pakalka, S., Kučas, S., Masys, K. A., et al. 2018, Phys. Rev. A, 97, 012708

Paunzen, E. 2015, VizieR Online Data Catalog: III/58

Pereira, C., Wesemael, F., \& Bergeron, P. 2006, Balt. Astron., 15, 123

Peterson, A. V. 1970, PhD Thesis, California Institute of Technology, California, USA

Pinnington, E. H., Bahr, J. L., Kernahan, J. A., \& Irwin, D. J. G. 1981, J. Phys. B At. Mol. Phys., 14, 1291

Pinnington, E. H., Ansbacher, W., Kernahan, J. A., Gosselin, R. N., \& Bahr, J. L. 1985a, J. Opt. Soc. Am. B Opt. Phys., 2, 1653 
Pinnington, E. H., Ansbacher, W., Kernahan, J. A., \& Inamdar, A. S. 1985b, J. Opt. Soc. Am. B Opt. Phys., 2, 331

Pinnington, E. H., Ansbacher, W., Kernahan, J. A., Ge, Z.-Q., \& Inamdar, A. S. 1988, Nucl. Instrum. Methods Phys. Res. B, 31, 206

Rauch, T., Werner, K., Quinet, P., \& Kruk, J. W. 2014, A\&A, 566, A10

Rauch, T., Quinet, P., Hoyer, D., et al. 2015, Tuebingen Oscillator Strengths Service Form Interface, VO resource provided by the GAVO Data Center

Rauch, T., Quinet, P., Hoyer, D., et al. 2016a, A\&A, 587, A39

Rauch, T., Quinet, P., Hoyer, D., et al. 2016b, A\&A, 590, A128

Rauch, T., Gamrath, S., Quinet, P., et al. 2017a, A\&A, 599, A142

Rauch, T., Quinet, P., Knörzer, M., et al. 2017b, A\&A, 606, A105

Redfors, A. 1991, A\&A, 249, 589

Safronova, U. I., \& Johnson, W. R. 2004, Phys. Rev. A, 69, 052511

Safronova, M. S., \& Safronova, U. I. 2013, Phys. Rev. A, 87, 062509

Safronova, U. I., Savukov, I. M., Safronova, M. S., \& Johnson, W. R. 2003, Phys. Rev. A, 68, 062505

Safronova, M. S., Safronova, U. I., \& Clark, C. W. 2014, Phys. Rev. A, 90, 032512
Schindewolf, M., Németh, P., Heber, U., et al. 2018, A\&A, 620, A36 Schork, M. 2018, Teacher's Thesis, Friedrich-Alexander University ErlangenNürnberg, Germany

Schwab, J. 2018, MNRAS, 476, 5303

Simon, K. P., Gruschinske, J., Hunger, K., \& Kudritzki, R. P. 1980, in Ultraviolet observations of Quasars, eds. B. Battrick \& J. Mort, ESA SP, 157, 305 Spencer Jones, J. H. 1985, MNASSA, 44, 33

Stroeer, A., Heber, U., Lisker, T., et al. 2007, A\&A, 462, 269

Tomley, L. 1970, ApJ, 162, 239

Unglaub, K. 2008, A\&A, 486, 923

Unglaub, K. 2010, AIP Conf. Ser., 1273, 251

van Hoof, P. 2017, The Atomic Line List v2.05b21, http://www . pa.uky.edu/ $\sim$ peter/newpage/, accessed: 2018-11-05

Wamsteker, W., Skillen, I., Ponz, J., et al. 2000, Ap\&SS, 273, 155

Werner, K., Rauch, T., Knörzer, M., \& Kruk, J. W. 2018, A\&A, 614, A96

Zhang, W., Palmeri, P., Quinet, P., \& Biémont, É. 2013, ApJ, 551, A136

Zhang, X., Hall, P. D., Jeffery, C. S., \& Bi, S. 2017, ApJ, 835, 242

Zhang, X., \& Jeffery, C. S. 2012, MNRAS, 419, 452 


\section{Appendix A: Additional material}

\section{A.1. Spectroscopic and photometric data}

Table A.1. Spectra used in our analysis.

\begin{tabular}{|c|c|c|c|c|c|}
\hline Star & Instrument & Dataset & Range $(\AA)$ & Exp. (s) & $R$ \\
\hline \multirow[t]{21}{*}{ HD 127493} & \multirow[t]{3}{*}{ FEROS } & ADP.2016-09-21T07:07:18.680 & $3527.9-9217.7$ & 600 & \multirow[t]{3}{*}{48000} \\
\hline & & ADP.2016-09-21T07:07:18.736 & $3527.9-9217.7$ & 300 & \\
\hline & & ADP.2016-09-21T07:07:18.686 & $3527.9-9217.7$ & 300 & \\
\hline & \multirow[t]{4}{*}{ IUE LWR } & LWR03587HS & $1850.0-3350.0$ & 5400 & \multirow[t]{4}{*}{10000} \\
\hline & & LWR04198HL & $1850.0-3350.0$ & 4450 & \\
\hline & & LWR06702HL & $1850.0-3350.0$ & 5400 & \\
\hline & & LWR07211HL & $1850.0-3350.0$ & 2950 & \\
\hline & \multirow[t]{10}{*}{ GHRS G160M } & Z2H60107T & $1222.6-1258.8$ & 462 & \multirow[t]{10}{*}{$0.07 \AA$} \\
\hline & & $\mathrm{Z} 2 \mathrm{H} 60109 \mathrm{~T}$ & $1254.9-1291.0$ & 462 & \\
\hline & & Z2H6010BT & $1285.6-1321.6$ & 462 & \\
\hline & & Z2H6010DT & $1317.7-1353.6$ & 462 & \\
\hline & & Z2H6010FT & $1349.7-1385.5$ & 517 & \\
\hline & & $\mathrm{Z} 2 \mathrm{H} 6010 \mathrm{HT}$ & $1383.0-1418.8$ & 598 & \\
\hline & & Z2H6010JT & $1414.9-1450.5$ & 517 & \\
\hline & & Z2H6010LT & $1532.5-1567.7$ & 653 & \\
\hline & & Z2H6010OT & $1623.2-1658.1$ & 462 & \\
\hline & & Z2H6010QT & $1713.0-1747.6$ & 462 & \\
\hline & \multirow[t]{4}{*}{ IUE SWP } & SWP04071HS & $1150.0-1980.0$ & 5880 & \multirow[t]{4}{*}{10000} \\
\hline & & SWP04860HL & $1150.0-1980.0$ & 3000 & \\
\hline & & SWP07695HL & $1150.0-1980.0$ & 4500 & \\
\hline & & SWP08276HL & $1150.0-1980.0$ & 3930 & \\
\hline \multirow[t]{11}{*}{$\mathrm{HZ} 44$} & \multirow[t]{6}{*}{ HIRES } & HI.20050810.20686 & $3214.0-5990.0$ & 600 & \multirow[t]{6}{*}{36000} \\
\hline & & HI.20050810.21381 & $3890.0-6732$ & 600 & \\
\hline & & HI.20050812.21565 & $3214.0-5990$ & 500 & \\
\hline & & HI.20070504.38715 & $3022.0-5800$ & 900 & \\
\hline & & HI.20160203.58141 & $4716.0-7580$ & 600 & \\
\hline & & HI.20160401.55323 & $3128.0-5947$ & 600 & \\
\hline & \multirow[t]{2}{*}{ IUE SWP } & SWP16294HL & $1150.0-1980.0$ & 9600 & \multirow[t]{2}{*}{10000} \\
\hline & & SWP17350HL & $1150.0-1980.0$ & 14820 & \\
\hline & FUSE MDRS & p3020401000 & $904.3-1188.4$ & 5919 & 19000 \\
\hline & FUSE LWRS & m1080401000 & $904.3-1188.4$ & 4679 & 17000 \\
\hline & FUSE LWRS & s5051901000 & $904.3-1188.4$ & 3937 & \\
\hline
\end{tabular}

\section{A.2. Comparison with literature}

Figure A.1 shows the comparison of abundances determined in this paper with literature values for HZ 44 and HD 127493. The only previous metal analysis of HZ 44 was performed by Peterson (1970) using the curve-of-growth method. Their results for $\mathrm{C}, \mathrm{N}, \mathrm{O}, \mathrm{Ne}, \mathrm{Mg}, \mathrm{Al}, \mathrm{Si}$, and $\mathrm{S}$ are consistent with the values presented in this paper considering $1 \sigma$ uncertainties. Only their $\mathrm{H}$ abundance (based on early ATLAS model atmospheres) and Fe abundance (based on three weak optical Fe III lines with at the time uncertain oscillator strengths) are overestimated compared to ours.

Peterson (1970) also performed a curve-of-growth analysis of optical spectra for HD 127493 (including C, N, Mg, and Si), which agrees well with the abundances derived here. A similar analysis was performed by Tomley (1970); his abundance results for $\mathrm{C}$ and $\mathrm{Ne}$ are higher by about $1 \mathrm{dex}$, while the abundances of $\mathrm{N}, \mathrm{Mg}$, and Si match within the respective uncertainties. $\mathrm{C}$ and $\mathrm{Si}$ abundance determinations from early NLTE models by Bauer \& Husfeld (1995) are higher by $\sim 0.5$ dex whereas their $\mathrm{N}$ and $\mathrm{Mg}$ abundances match well. The $\mathrm{C}$ and $\mathrm{N}$ abundances derived by Hirsch (2009) are consistent with our results.

\section{A.3. Abundances and stellar spectra}

This section presents our final abundance values (Table A.5) as well as a comparison between the full observed and final synthetic spectra of HZ 44 and HD 127493 (Figs. A.2-A.6). In the synthetic spectra, elements with upper limits only are included at their upper limit. The synthetic spectra are convolved with a Gaussian kernel (constant for GHRS, but wavelength-dependent for all echelle spectrographs) to match the resolution of the respective spectrograph. The strongest photospheric metal lines are labeled with magenta marks, interstellar lines are labeled with green marks. At the bottom of each spectral range we also show the residual between the observation and our final model. A proper normalization of the HIRES spectrum of HZ 44 was only possible using the final synthetic spectrum as a template. Thus the shape of broad hydrogen and helium lines in the HIRES spectra is adjusted during the normalization procedure to fit the shape of the synthetic spectrum. However, the shape of the sharp metal lines, which are of interest in the HIRES spectra, are not affected by the normalization procedure. The optical spectra of HZ 44 and HD 127493 are shown up to $6710 \AA$ since the number of metal lines at longer wavelengths is very limited. 
Table A.2. Radial velocity measurements for HZ 44 and HIRES spectra used.

\begin{tabular}{|c|c|c|}
\hline Time (YYYY-MM-DD hh:mm) & Number of considered lines & $v_{\mathrm{rad}}\left(\mathrm{km} \mathrm{s}^{-1}\right)$ \\
\hline $1995-07-02$ 05:26 & 10 & $12.4 \pm 0.8$ \\
\hline 1995-07-02 05:33 & 10 & $12.9 \pm 0.7$ \\
\hline $1995-07-02$ 05:40 & 10 & $13.0 \pm 0.6$ \\
\hline $1996-05-2607: 50$ & 10 & $12.8 \pm 0.6$ \\
\hline $1997-07-13$ 05:54 & 19 & $13.0 \pm 0.6$ \\
\hline $1998-05-19$ 05:33 & 25 & $11.9 \pm 0.7$ \\
\hline $1999-02-14$ 15:45 & 20 & $12.6 \pm 0.5$ \\
\hline 2000-02-05 16:10 & 5 & $12.0 \pm 1.0$ \\
\hline 2001-03-02 16:04 & 23 & $12.2 \pm 0.6$ \\
\hline 2002-02-03 16:18 & 10 & $12.9 \pm 0.7$ \\
\hline 2002-08-04 05:26 & 18 & $12.7 \pm 0.9$ \\
\hline $2005-08-10$ 05:45 & 27 & $12.6 \pm 0.9$ \\
\hline $2005-08-12 \quad 05: 59$ & 27 & $11.9 \pm 0.6$ \\
\hline 2005-08-12 08:10 & 22 & $12.7 \pm 0.6$ \\
\hline 2006-06-18 05:37 & 29 & $13.2 \pm 0.7$ \\
\hline 2006-06-18 05:39 & 27 & $13.3 \pm 0.8$ \\
\hline 2006-06-18 05:42 & 26 & $13.2 \pm 0.8$ \\
\hline 2007-05-04 10:45 & 17 & $13.2 \pm 0.5$ \\
\hline $2008-07-1105: 53$ & 10 & $12.7 \pm 0.9$ \\
\hline 2008-07-11 06:00 & 10 & $12.4 \pm 0.8$ \\
\hline 2009-07-13 06:56 & 19 & $12.9 \pm 0.7$ \\
\hline 2012-01-04 16:14 & 17 & $12.4 \pm 0.5$ \\
\hline 2012-01-04 16:17 & 17 & $12.3 \pm 0.5$ \\
\hline $2013-05-05$ 05:21 & 22 & $13.0 \pm 0.7$ \\
\hline $2013-05-07$ 05:24 & 25 & $12.7 \pm 0.7$ \\
\hline 2015-04-09 15:34 & 12 & $12.0 \pm 0.6$ \\
\hline $2015-04-11$ 15:24 & 19 & $12.4 \pm 0.6$ \\
\hline
\end{tabular}

Table A.3. Photometric data used for the SED-fit of HZ 44.

\begin{tabular}{|c|c|c|c|c|c|}
\hline System & Passband & Magnitude & Uncertainty & Type & Reference \\
\hline 2MASS & $H$ & 12.569 & 0.023 & Magnitude & (Cutri et al. 2003, 2MASS: II/246/out) \\
\hline 2MASS & $J$ & 12.386 & 0.022 & Magnitude & (Cutri et al. 2003, 2MASS: II/246/out) \\
\hline 2MASS & $K$ & 12.672 & 0.027 & Magnitude & (Cutri et al. 2003, 2MASS: II/246/out) \\
\hline Stroemgren & $H_{\beta}$ & 2.617 & & Color & (Paunzen $2015, \mathrm{~J} / \mathrm{A}+\mathrm{A} / 580 / \mathrm{A} 23 /$ catalog) \\
\hline Stroemgren & $b-y$ & -0.151 & & Color & (Paunzen 2015, J/A+A/580/A23/catalog) \\
\hline Stroemgren & $m 1$ & 0.104 & 0.020 & Color & (Paunzen 2015, J/A+A/580/A23/catalog) \\
\hline Stroemgren & $y$ & 11.715 & 0.007 & Magnitude & (Paunzen 2015, J/A+A/580/A23/catalog) \\
\hline UKIDSS & $H$ & 12.560 & 0.002 & Magnitude & (Lawrence et al. 2013, UKIDSS DR9: II/319/las9) \\
\hline UKIDSS & $J$ & 12.400 & 0.001 & Magnitude & (Lawrence et al. 2013, UKIDSS DR9: II/319/las9) \\
\hline UKIDSS & $K$ & 12.687 & 0.002 & Magnitude & (Lawrence et al. 2013, UKIDSS DR9: II/319/las9) \\
\hline UKIDSS & $Y$ & 12.276 & 0.001 & Magnitude & (Lawrence et al. 2013, UKIDSS DR9: II/319/las9) \\
\hline WISE & $W 1$ & 12.750 & 0.023 & Magnitude & (Cutri et al. 2012, AllWISE: II/328/allwise) \\
\hline WISE & $W 2$ & 12.830 & 0.025 & Magnitude & (Cutri et al. 2012, AllWISE: II/328/allwise) \\
\hline IUE box & $1300-1800 \AA$ & 7.903 & 0.020 & Magnitude & (Wamsteker et al. 2000, VI/110/inescat, SWP03432LL) \\
\hline IUE box & $2000-2500 \AA$ & 8.609 & 0.020 & Magnitude & (Wamsteker et al. 2000, VI/110/inescat, LWR03017LL) \\
\hline IUE box & $2500-3000 \AA$ & 9.047 & 0.020 & Magnitude & (Wamsteker et al. 2000, VI/110/inescat, LWR03017LL) \\
\hline Gaia & $\mathrm{G}$ & 11.6350 & 0.001 & Magnitude & (Gaia Collaboration 2018, I/345/gaia2) \\
\hline Gaia & GBP & 11.3913 & 0.007 & Magnitude & (Gaia Collaboration 2018, I/345/gaia2) \\
\hline Gaia & GRP & 11.9377 & 0.001 & Magnitude & (Gaia Collaboration 2018, I/345/gaia2) \\
\hline Johnson & $V-I$ & -0.322 & 0.002 & Color & (Landolt \& Uomoto 2007, J/AJ/133/768/table4) \\
\hline Johnson & $R-I$ & -0.181 & 0.001 & Color & (Landolt \& Uomoto 2007, J/AJ/133/768/table4) \\
\hline Johnson & $V-R$ & -0.141 & 0.001 & Color & (Landolt \& Uomoto 2007, J/AJ/133/768/table4) \\
\hline Johnson & $B-V$ & -0.291 & 0.001 & Color & (Landolt \& Uomoto 2007, J/AJ/133/768/table4) \\
\hline Johnson & $U-B$ & -1.196 & 0.003 & Color & (Landolt \& Uomoto 2007, J/AJ/133/768/table4) \\
\hline Johnson & $V$ & 11.673 & 0.002 & Magnitude & (Landolt \& Uomoto 2007, J/AJ/133/768/table4) \\
\hline
\end{tabular}


Table A.4. Photometric data used for the SED-fit of HD 127493.

\begin{tabular}{|c|c|c|c|c|c|}
\hline System & Passband & Magnitude & Uncertainty & Type & Reference \\
\hline 2MASS & $H$ & 10.816 & 0.028 & Magnitude & (Cutri et al. 2003, 2MASS: II/246/out) \\
\hline 2MASS & $J$ & 10.641 & 0.023 & Magnitude & (Cutri et al. 2003, 2MASS: II/246/out) \\
\hline 2MASS & $K$ & 10.907 & 0.025 & Magnitude & (Cutri et al. 2003, 2MASS: II/246/out) \\
\hline Johnson & $B-V$ & -0.234 & & Color & (Mermilliod 2006, II/168/ubvmeans) \\
\hline Johnson & $U-B$ & -1.17 & & Color & (Mermilliod 2006, II/168/ubvmeans) \\
\hline Johnson & $V$ & 10.05 & & Magnitude & (Mermilliod 2006, II/168/ubvmeans) \\
\hline Stroemgren & $H_{\beta}$ & 2.57 & & Color & (Paunzen 2015, J/A+A/580/A23/catalog) \\
\hline Stroemgren & $b-y$ & -0.114 & 0.003 & Color & (Paunzen 2015, J/A+A/580/A23/catalog) \\
\hline Stroemgren & $c 1$ & -0.214 & 0.013 & Color & (Paunzen 2015, J/A+A/580/A23/catalog) \\
\hline Stroemgren & $m 1$ & 0.048 & 0.002 & Color & (Paunzen 2015, J/A+A/580/A23/catalog) \\
\hline Stroemgren & $y$ & 10.035 & 0.009 & Magnitude & (Paunzen 2015, J/A+A/580/A23/catalog) \\
\hline WISE & W1 & 10.954 & 0.023 & Magnitude & (Cutri et al. 2012, AllWISE: II/328/allwise) \\
\hline WISE & W2 & 11.045 & 0.021 & Magnitude & (Cutri et al. 2012, AllWISE: II/328/allwise) \\
\hline IUE box & $1300-1800$ & 6.306 & 0.02 & Magnitude & (Wamsteker et al. 2000, VI/110/inescat, SWP08275LL) \\
\hline IUE box & $2000-2500$ & 7.164 & 0.02 & Magnitude & (Wamsteker et al. 2000, VI/110/inescat, LWR07210LL) \\
\hline IUE box & $2500-3000$ & 7.547 & 0.02 & Magnitude & (Wamsteker et al. 2000, VI/110/inescat, LWR07210LL) \\
\hline Gaia $G$ & & 9.9636 & 0.0011 & Magnitude & (Gaia Collaboration 2018, I/345/gaia2) \\
\hline Gaia GBP & & 9.8227 & 0.0038 & Magnitude & (Gaia Collaboration 2018, I/345/gaia2) \\
\hline Gaia GRP & & 10.2446 & 0.0015 & Magnitude & (Gaia Collaboration 2018, I/345/gaia2) \\
\hline Johnson & $B-V$ & -0.258 & & Color & Menzies et al. (1990) \\
\hline Johnson & $U-B$ & -1.165 & & Color & Menzies et al. (1990) \\
\hline Johnson & $V$ & 10.01 & & Magnitude & Menzies et al. (1990) \\
\hline Johnson & $B-V$ & -0.269 & & Color & Kilkenny et al. (1998) \\
\hline Johnson & $U-B$ & -1.184 & & Color & Kilkenny et al. (1998) \\
\hline Johnson & $V-R$ & -0.115 & & Color & Kilkenny et al. (1998) \\
\hline Johnson & $V-I$ & -0.276 & & Color & Kilkenny et al. (1998) \\
\hline Johnson & $V$ & 10.039 & & Magnitude & Kilkenny et al. (1998) \\
\hline
\end{tabular}




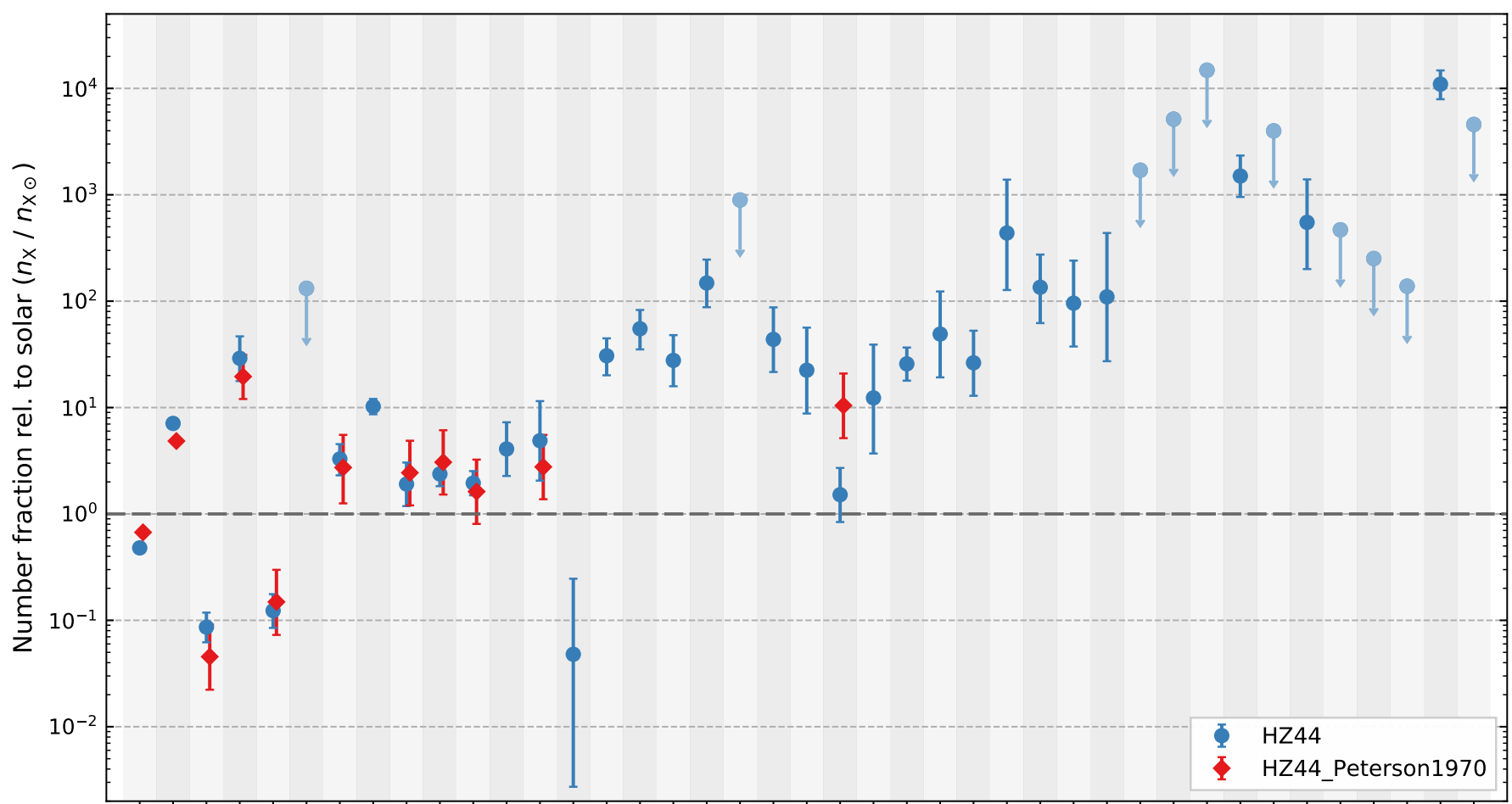

H He C N O F NeNaMgAl Si P S Cl Ar K Ca Ti V CrMnFeCo NiCuZnGaGeAsSe Kr Sr Y ZrMoSnSbTeXePbTh

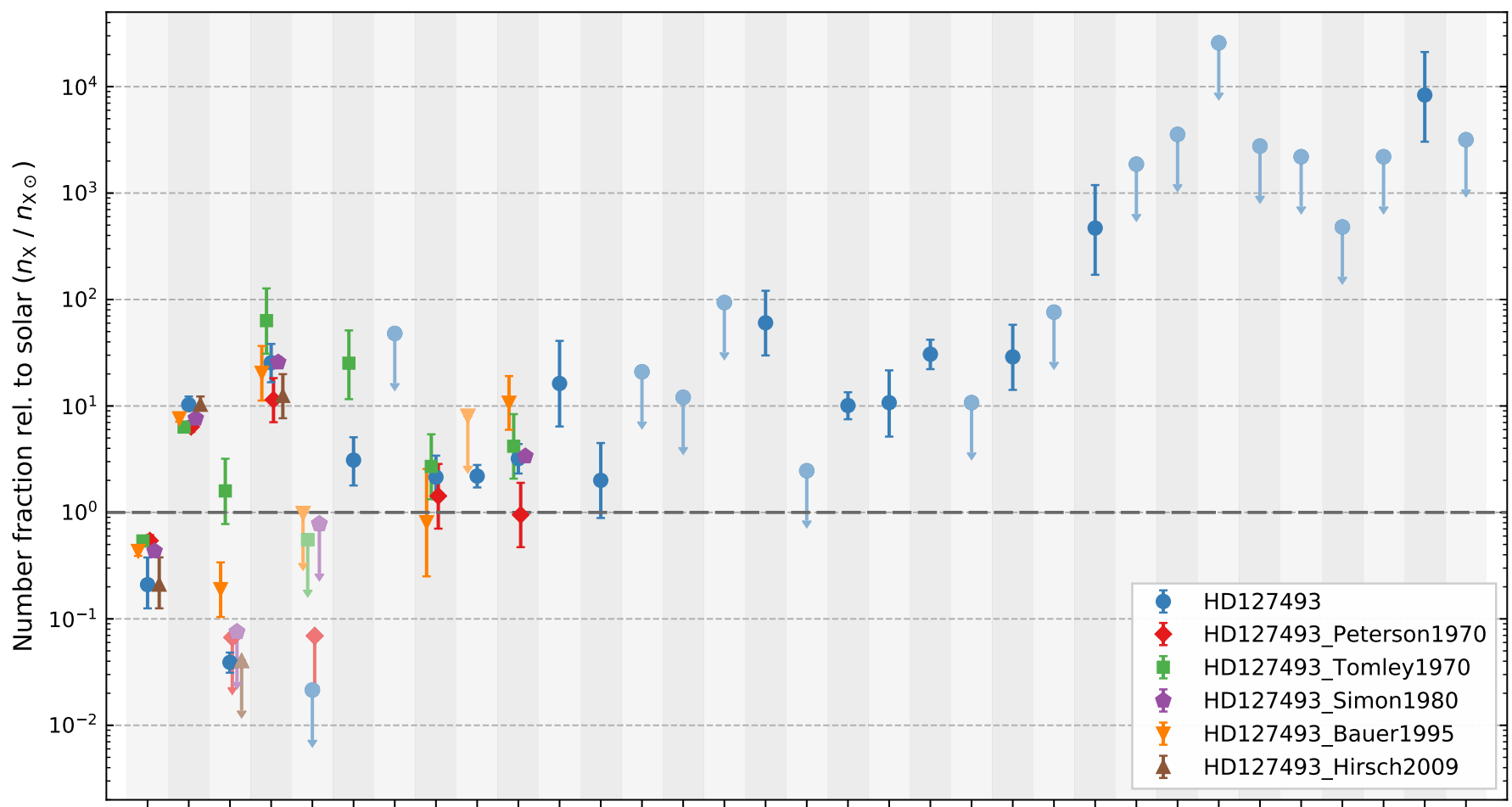

H He C N O NeNaMg Al Si P S Ar Ca Ti Cr Mn Fe Co Ni Cu Zn GaGe Kr Sr Y Zr Mo Sn Te Pb Th

Fig. A.1. Same as Fig. 13 but for the comparison of abundances derived in this paper with literature values. 
Table A.5. Abundances of HZ 44 and HD 127493 as derived from visual/UVA and FUV data.

\begin{tabular}{|c|c|c|c|c|c|c|c|c|}
\hline \multirow[b]{2}{*}{ Element } & \multicolumn{2}{|c|}{ Abundance $\left(\log n_{\mathrm{X}} / n_{\mathrm{H}}\right)$} & \multicolumn{2}{|c|}{ Mass fraction $\left(\beta_{\mathrm{X}}\right)$} & \multicolumn{2}{|c|}{ Number fraction $\left(\log n_{\mathrm{X}}\right)$} & \multicolumn{2}{|c|}{ Abundance $\left(\log n_{\mathrm{X}} / n_{\mathrm{X}, \odot}\right)$} \\
\hline & $\mathrm{HZ} 44$ & HD 127493 & HZ 44 & HD 127493 & $\mathrm{HZ} 44$ & HD 127493 & $\mathrm{HZ} 44$ & HD 127493 \\
\hline $\mathrm{H}$ & $0.00_{-0.00}^{+0.00}$ & $0.00_{-0.00}^{+0.00}$ & $-0.79_{-0.03}^{+0.03}$ & $-1.26_{-0.21}^{+0.25}$ & $-0.36_{-0.03}^{+0.03}$ & $-0.71_{-0.22}^{+0.26}$ & $-0.32_{-0.03}^{+0.03}$ & $-0.68_{-0.22}^{+0.26}$ \\
\hline $\mathrm{He}$ & $0.10_{-0.05}^{+0.05}$ & $0.62_{-0.30}^{+0.30}$ & $-0.09_{-0.01}^{+0.01}$ & $-0.03_{-0.01}^{+0.02}$ & $-0.26_{-0.02}^{+0.02}$ & $-0.09_{-0.04}^{+0.08}$ & $0.85_{-0.02}^{+0.02}$ & $1.01_{-0.05}^{+0.08}$ \\
\hline $\mathrm{C}$ & $-4.31_{-0.13}^{+0.13}$ & $-4.30_{-0.08}^{+0.08}$ & $-4.02_{-0.13}^{+0.13}$ & $-4.48_{-0.09}^{+0.11}$ & $-4.67_{-0.13}^{+0.02}$ & $\begin{array}{r}-5.01_{-0.08}^{+0.08} \\
-0.08\end{array}$ & $-1.06_{-0.14}^{+0.02}$ & $-1.41_{-0.10}^{+0.09}$ \\
\hline $\mathrm{N}$ & $-2.39_{-0.20}^{+0.20}$ & $-2.08_{-0.17}^{+0.17}$ & $-2.03_{-0.20}^{+0.20}$ & $-2.19_{-0.18}^{+0.18}$ & $-2.74_{-0.20}^{+0.20}$ & $-2.80_{-0.17}^{+0.17}$ & $1.46_{-0.21}^{+0.21}$ & $1.41_{-0.18}^{+0.18}$ \\
\hline $\mathrm{O}$ & $-3.90_{-0.15}^{+0.15}$ & $<-4.30^{+0.20}$ & $-3.49_{-0.15}^{+0.15}$ & $<-4.35^{+0.21}$ & $-4.26_{-0.15}^{+0.15}$ & $<-5.01^{+0.20}$ & $-0.91_{-0.16}^{+0.16}$ & $<-1.67^{+0.20}$ \\
\hline $\mathrm{F}$ & $<-5.00^{+0.40}$ & & $<-4.51^{+0.40}$ & & $<-5.36^{+0.40}$ & & $<2.12^{+0.45}$ & \\
\hline $\mathrm{Ne}$ & $-3.23_{-0.11}^{+0.11}$ & $-2.90_{-0.20}^{+0.20}$ & $-2.72_{-0.11}^{+0.11}$ & $-2.85_{-0.21}^{+0.21}$ & $-3.59_{-0.11}^{+0.11}$ & $-3.61_{-0.20}^{+0.20}$ & $0.52_{-0.15}^{+0.14}$ & $0.49_{-0.24}^{+0.21}$ \\
\hline $\mathrm{Na}$ & $-4.43_{-0.06}^{+0.06}$ & $<-3.40^{+0.30}$ & $-3.86_{-0.06}^{+0.06}$ & $<-3.29^{+0.30}$ & $-4.79_{-0.06}^{+0.06}$ & $<-4.11^{+0.30}$ & $1.01_{-0.07}^{+0.07}$ & $<1.68^{+0.30}$ \\
\hline $\mathrm{Mg}$ & $-3.80_{-0.20}^{+0.20}$ & $-3.39_{-0.20}^{+0.20}$ & $-3.20_{-0.20}^{+0.20}$ & $-3.26_{-0.21}^{+0.21}$ & $-4.16_{-0.20}^{+0.20}$ & $-4.10_{-0.20}^{+0.20}$ & $0.28_{-0.21}^{+0.20}$ & $0.33_{-0.21}^{+0.20}$ \\
\hline $\mathrm{Al}$ & $-4.86_{-0.11}^{+0.11}$ & $-4.53_{-0.10}^{+0.10}$ & $-4.21_{-0.11}^{+0.11}$ & $-4.35_{-0.11}^{+0.12}$ & $-5.21_{-0.11}^{+0.11}$ & $-5.24_{-0.10}^{+0.10}$ & $0.38_{-0.11}^{+0.11}$ & $0.34_{-0.11}^{+0.10}$ \\
\hline $\mathrm{Si}$ & $-3.88_{-0.11}^{+0.11}$ & $-3.31_{-0.14}^{+0.14}$ & $-3.22_{-0.11}^{+0.11}$ & $-3.11_{-0.14}^{+0.15}$ & $-4.24_{-0.11}^{+0.11}$ & $-4.02_{-0.13}^{+0.14}$ & $0.29_{-0.12}^{+0.11}$ & $0.51_{-0.14}^{+0.14}$ \\
\hline $\mathrm{P}$ & $-5.66_{-0.25}^{+0.25}$ & $-4.70_{-0.40}^{+0.40}$ & $-4.96_{-0.25}^{+0.25}$ & $-4.46_{-0.41}^{+0.40}$ & $-6.02_{-0.25}^{+0.25}$ & $-5.41_{-0.40}^{+0.40}$ & $0.61_{-0.25}^{+0.25}$ & $1.21_{-0.40}^{+0.40}$ \\
\hline S & $-3.87_{-0.37}^{+0.37}$ & $-3.90_{-0.35}^{+0.35}$ & $-3.16_{-0.37}^{+0.37}$ & $-3.65_{-0.36}^{+0.35}$ & $-4.23_{-0.37}^{+0.37}$ & $-4.61_{-0.35}^{+0.35}$ & $0.69_{-0.38}^{+0.37}$ & $0.30_{-0.35}^{+0.35}$ \\
\hline $\mathrm{Cl}$ & $-7.50_{-0.70}^{+0.70}$ & & $-6.74_{-0.70}^{+0.70}$ & & $-7.86_{-0.70}^{+0.70}$ & & $\begin{array}{r}-1.32_{-1.25}^{+0.78} \\
-0.71\end{array}$ & \\
\hline $\mathrm{Ar}$ & $-3.79_{-0.11}^{+0.11}$ & $<-3.60^{+0.20}$ & $-2.98_{-0.11}^{+0.11}$ & $<-3.25^{+0.21}$ & $-4.15_{-0.11}^{+0.11}$ & $<-4.31^{+0.20}$ & $1.49_{-0.18}^{+0.16}$ & $<1.32^{+0.23}$ \\
\hline K & $-4.91_{-0.16}^{+0.16}$ & & $-4.11_{-0.16}^{+0.16}$ & & $-5.27_{-0.16}^{+0.16}$ & & $1.74_{-0.19}^{+0.18}$ & \\
\hline $\mathrm{Ca}$ & $-3.90_{-0.24}^{+0.24}$ & $<-3.90^{+0.20}$ & $-3.08_{-0.24}^{+0.24}$ & $<-3.55^{+0.21}$ & $-4.25_{-0.24}^{+0.24}$ & $<-4.61^{+0.20}$ & $1.44_{-0.24}^{+0.24}$ & $<1.08^{+0.20}$ \\
\hline $\mathrm{Ti}$ & $-4.56_{-0.22}^{+0.22}$ & $<-4.40^{+0.25}$ & $-3.67_{-0.22}^{+0.22}$ & $<-3.98^{+0.26}$ & $-4.92_{-0.22}^{+0.22}$ & $<-5.11^{+0.25}$ & $2.17_{-0.23}^{+0.22}$ & $<1.97^{+0.25}$ \\
\hline $\mathrm{V}$ & $<-4.80^{+0.40}$ & & $<-3.88^{+0.40}$ & & $<-5.16^{+0.40}$ & & $<2.95^{+0.40}$ & \\
\hline $\mathrm{Cr}$ & $-4.40_{-0.30}^{+0.30}$ & $-3.90_{-0.30}^{+0.30}$ & $-3.47_{-0.30}^{+0.30}$ & $-3.44_{-0.31}^{+0.30}$ & $-4.76_{-0.30}^{+0.30}$ & $-4.61_{-0.30}^{+0.30}$ & $1.64_{-0.31}^{+0.30}$ & $1.78_{-0.31}^{+0.30}$ \\
\hline Mn & $\begin{array}{r}-0.50 \\
-4.90_{-0.40}^{+0.40}\end{array}$ & $<-5.50^{-0.30}$ & $\begin{array}{r}-0.30 \\
-3.95_{-0.40}^{+0.40}\end{array}$ & $<-5.02^{-0.30}$ & $\begin{array}{r}-0.30 \\
-5.26_{-0.40}^{+0.40}\end{array}$ & $<-6.21^{+0.30}$ & $1.35_{-0.41}^{+0.41}$ & $<0.39^{-0.31}$ \\
\hline $\mathrm{Fe}$ & $-4.00_{-0.25}^{+0.40}$ & $-2.82_{-0.12}^{+0.12}$ & $-3.04_{-0.25}^{+0.40}$ & $-2.33_{-0.13}^{+0.14}$ & $-4.36_{-0.25}^{+0.40}$ & $-3.53_{-0.12}^{+0.12}$ & $0.18_{-0.26}^{+0.25}$ & $1.00_{-0.13}^{+0.12}$ \\
\hline Co & $-5.60_{-0.50}^{+0.50}$ & $-5.30_{-0.30}^{+0.30}$ & $-4.62_{-0.50}^{+0.50}$ & $-4.78_{-0.31}^{+0.30}$ & $-5.96_{-0.50}^{+0.50}$ & $-6.01_{-0.30}^{+0.30}$ & $1.09_{-0.52}^{+0.50}$ & $1.03_{-0.32}^{+0.30}$ \\
\hline $\mathrm{Ni}$ & $-4.05_{-0.15}^{+0.15}$ & $-3.61_{-0.13}^{+0.13}$ & $-3.07_{-0.15}^{+0.15}$ & $-3.10_{-0.14}^{+0.15}$ & $-4.41_{-0.15}^{+0.15}$ & $-4.33_{-0.13}^{+0.13}$ & $1.41_{-0.16}^{+0.15}$ & $1.49_{-0.14}^{+0.14}$ \\
\hline $\mathrm{Cu}$ & $-5.80_{-0.40}^{+0.40}$ & $<-6.10^{+0.40}$ & $-4.79_{-0.40}^{+0.40}$ & $<-5.55^{-0.40}$ & $-6.16_{-0.40}^{+0.40}$ & $<-6.81^{-0.13}$ & $1.69_{-0.41}^{+0.40}$ & $<1.03^{+0.40}$ \\
\hline $\mathrm{Zn}$ & $\begin{array}{r}-0.40 \\
-5.70_{-0.30}^{+0.30}\end{array}$ & $-5.30_{-0.30}^{+0.30}$ & $\begin{array}{r}-0.40 \\
-4.67_{-0.30}^{+0.30}\end{array}$ & $-4.74_{-0.31}^{+0.30}$ & $\begin{array}{r}-0.40 \\
-6.06_{-0.30}^{+0.30}\end{array}$ & $-6.01_{-0.30}^{+0.30}$ & $1.42_{-0.31}^{+0.31}$ & $1.46_{-0.31}^{+0.30}$ \\
\hline $\mathrm{Ga}$ & $-6.00_{-0.50}^{+0.50}$ & $<-6.40^{+0.40}$ & $-4.95_{-0.50}^{+0.50}$ & $<-5.81^{+0.40}$ & $-6.36_{-0.50}^{+0.50}$ & $<-7.11^{+0.40}$ & $2.64_{-0.54}^{+0.50}$ & $<1.88^{+0.40}$ \\
\hline $\mathrm{Ge}$ & $-5.90_{-0.30}^{+0.30}$ & $-5.00_{-0.40}^{+0.40}$ & $-4.83_{-0.30}^{+0.30}$ & $-4.39_{-0.41}^{+0.40}$ & $\begin{array}{r}-0.56_{-0.30}^{+0.30} \\
-6.20\end{array}$ & $-5.71_{-0.40}^{+0.40}$ & $2.13_{-0.34}^{+0.34}$ & $2.67_{-0.44}^{+0.40}$ \\
\hline As & $-7.40_{-0.40}^{+0.40}$ & & $-6.31_{-0.40}^{+0.40}$ & & $-7.76_{-0.40}^{+0.40}$ & & $1.98_{-0.41}^{+0.40}$ & \\
\hline $\mathrm{Se}$ & $-6.30_{-0.60}^{+0.60}$ & & $-5.19_{-0.60}^{+0.60}$ & & $-6.66_{-0.60}^{+0.60}$ & & $2.04_{-0.61}^{+0.60}$ & \\
\hline $\mathrm{Kr}$ & $<-5.20^{+0.60}$ & $<-4.80^{+0.40}$ & $<-4.07^{+0.60}$ & $<-4.13^{+0.40}$ & $<-5.56^{+0.60}$ & $<-5.51^{+0.40}$ & $<3.23^{+0.60}$ & $<3.27^{+0.40}$ \\
\hline $\mathrm{Sr}$ & $<-5.10^{+0.60}$ & $<-4.90^{+0.30}$ & $<-3.95^{+0.60}$ & $<-4.21^{+0.30}$ & $<-5.46^{+0.60}$ & $<-5.61^{+0.30}$ & $<3.71^{+0.60}$ & $<3.55^{+0.30}$ \\
\hline $\mathrm{Y}$ & $<-5.30^{+0.20}$ & $<-4.70^{+0.30}$ & $<-4.14^{+0.20}$ & $<-4.01^{+0.30}$ & $<-5.66^{+0.20}$ & $<-5.41^{+0.30}$ & $<4.17^{+0.20}$ & $<4.41^{+0.30}$ \\
\hline $\mathrm{Zr}$ & $-5.92_{-0.19}^{+0.19}$ & $<-5.30^{+0.20}$ & $-4.75_{-0.19}^{+0.19}$ & $<-4.60^{+0.21}$ & $-6.28_{-0.19}^{+0.19}$ & $<-6.01^{+0.20}$ & $3.18_{-0.20}^{+0.19}$ & $<3.44^{+0.20}$ \\
\hline Mo & $<-6.20^{+0.40}$ & $<-6.10^{+0.40}$ & $<-5.01^{+0.40}$ & $<-5.37^{+0.40}$ & $<-6.56^{+0.40}$ & $<-6.81^{+0.40}$ & $<3.60^{-0.40}$ & $<3.34^{+0.40}$ \\
\hline $\mathrm{Sn}$ & $-6.90_{-0.40}^{+0.40}$ & $<-6.60^{+0.40}$ & $-5.61_{-0.40}^{+0.40}$ & $<-5.78^{+0.40}$ & $-7.26_{-0.40}^{+0.40}$ & $<-7.31^{+0.40}$ & $2.74_{-0.44}^{+0.40}$ & $<2.68^{+0.40}$ \\
\hline $\mathrm{Sb}$ & $<-8.00^{+0.50}$ & & $<-6.70^{+0.50}$ & & $<-8.36^{+0.50}$ & & $<2.67^{+0.50}$ & \\
\hline $\mathrm{Te}$ & $<-7.10^{+0.40}$ & $<-5.80^{+0.40}$ & $<-5.78^{+0.40}$ & $<-4.95^{+0.40}$ & $<-7.46^{+0.40}$ & $<-6.51^{+0.40}$ & $<2.40^{+0.40}$ & $<3.34^{+0.40}$ \\
\hline $\mathrm{Xe}$ & $<-7.30^{+0.40}$ & & $<-5.97^{+0.40}$ & & $<-7.66^{+0.40}$ & & $<2.14^{+0.40}$ & \\
\hline $\mathrm{Pb}$ & $-5.89_{-0.09}^{+0.09}$ & $-5.65_{-0.40}^{+0.40}$ & $-4.36_{-0.09}^{+0.09}$ & $-4.59_{-0.41}^{+0.40}$ & $-6.25_{-0.09}^{+0.09}$ & $-6.36_{-0.40}^{+0.40}$ & $4.04_{-0.14}^{+0.13}$ & $3.92_{-0.44}^{+0.40}$ \\
\hline Th & $<-8.00^{+0.30}$ & $<-7.80^{+0.30}$ & $<-6.42^{+0.30}$ & $<-6.69^{+0.30}$ & $<-8.36^{+0.30}$ & $<-8.51^{-0.30}$ & $<3.66^{-0.31}$ & $<3.50^{-0.44}$ \\
\hline
\end{tabular}

Notes. Abundances are given as the logarithmic number ratio of element $\mathrm{X}$ relative to hydrogen $\log n_{\mathrm{X}} / n_{\mathrm{H}}$, $\log$ arithmic mass fraction $\beta_{\mathrm{X}}$, logarithmic number fraction $\log n_{\mathrm{X}}$, and $\operatorname{logarithmic}$ number fraction relative to solar values $\log n_{\mathrm{X}} / n_{\mathrm{X}, \odot}$. Uncertainties are given as the standard deviation between single line fits. If an abundance was "fit by eye" the uncertainties are similarly estimated. The He abundance for HD 127493 is from Hirsch (2009). 


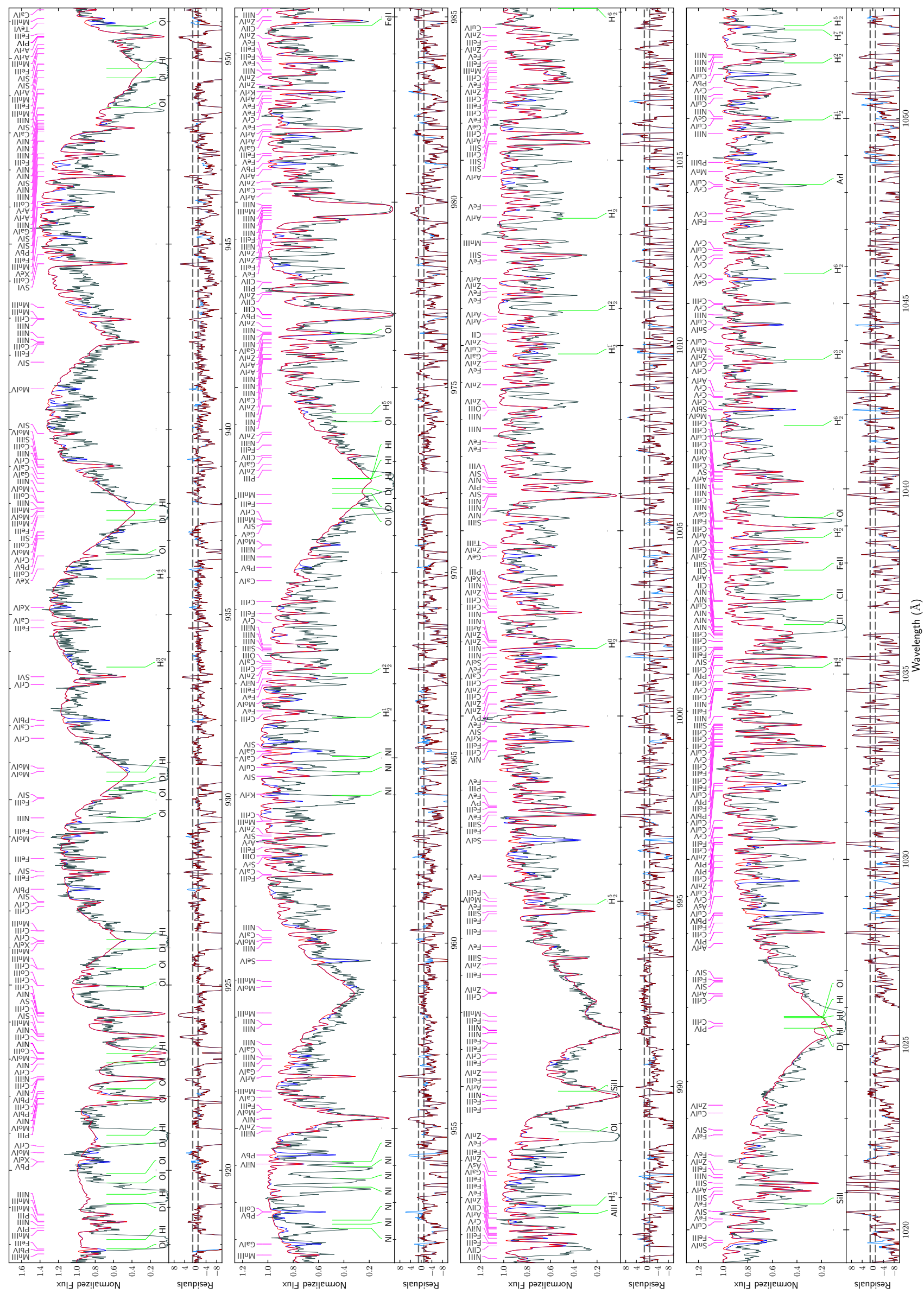

Fig. A.2. FUSE spectrum of HZ 44 (gray) and the final model (red, with heavy metals: blue). 

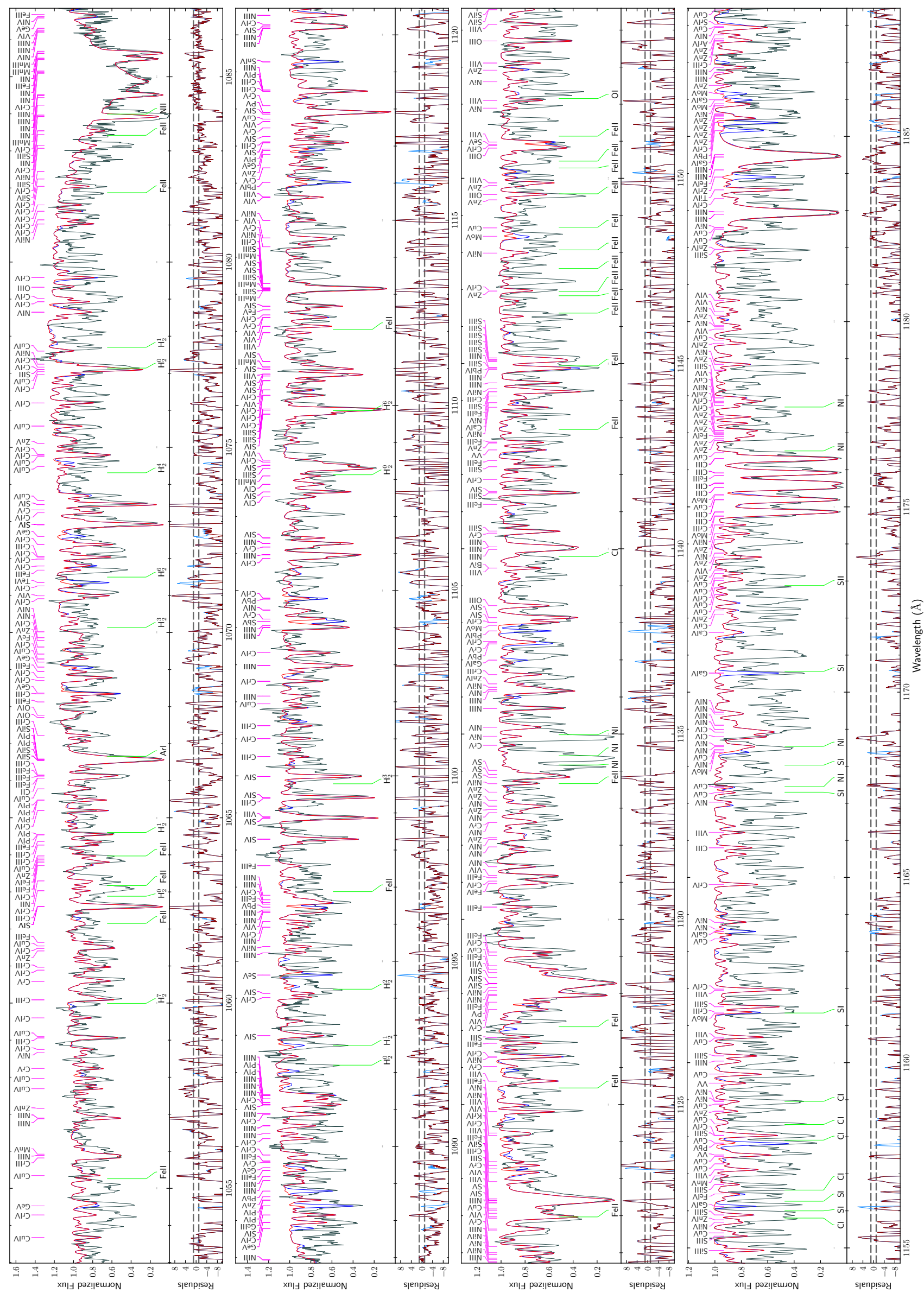

Fig. A.2. continued. 


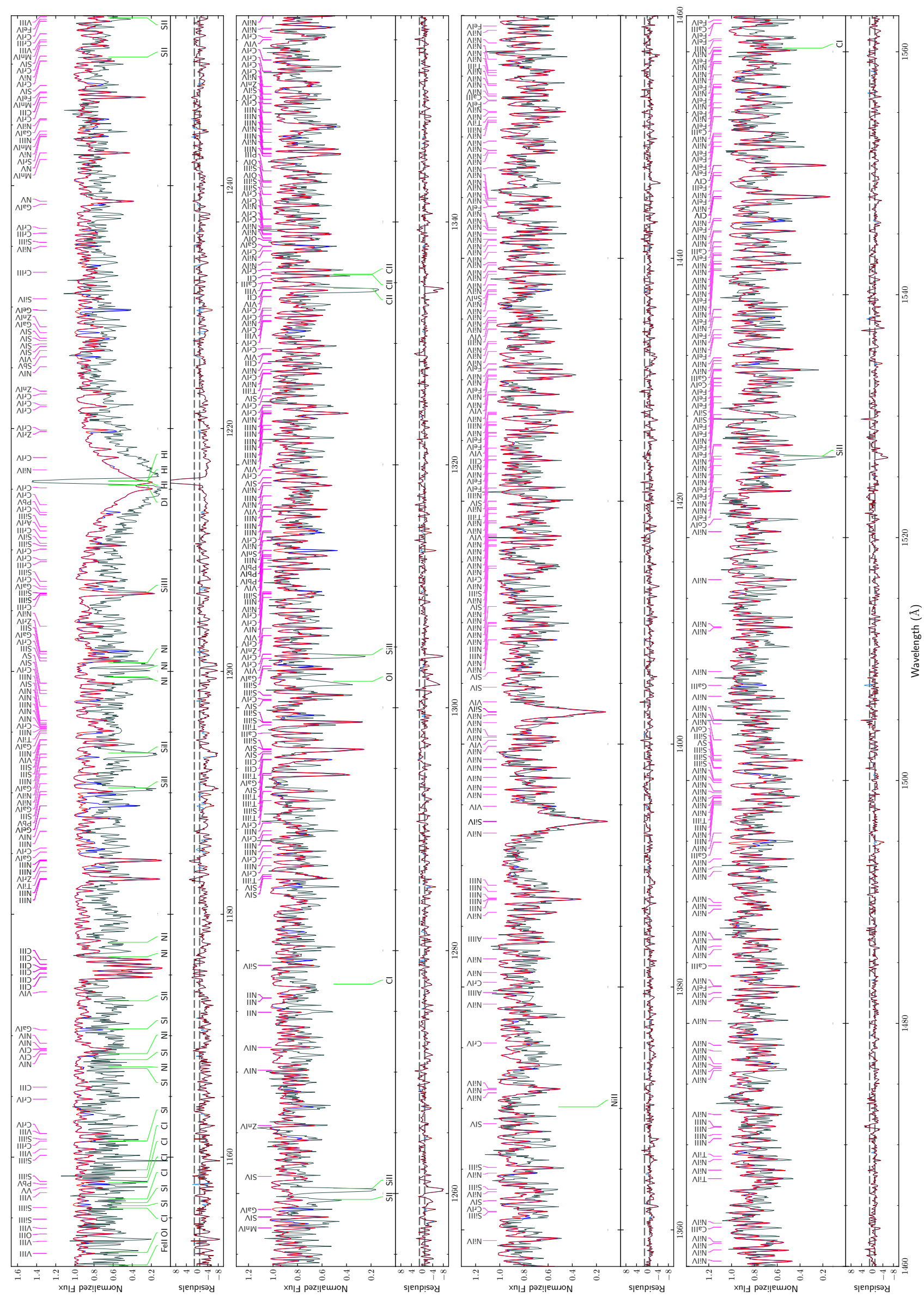

Fig. A.3. IUE spectrum of HZ 44 (gray) and the final model (red, with heavy metals: blue). 


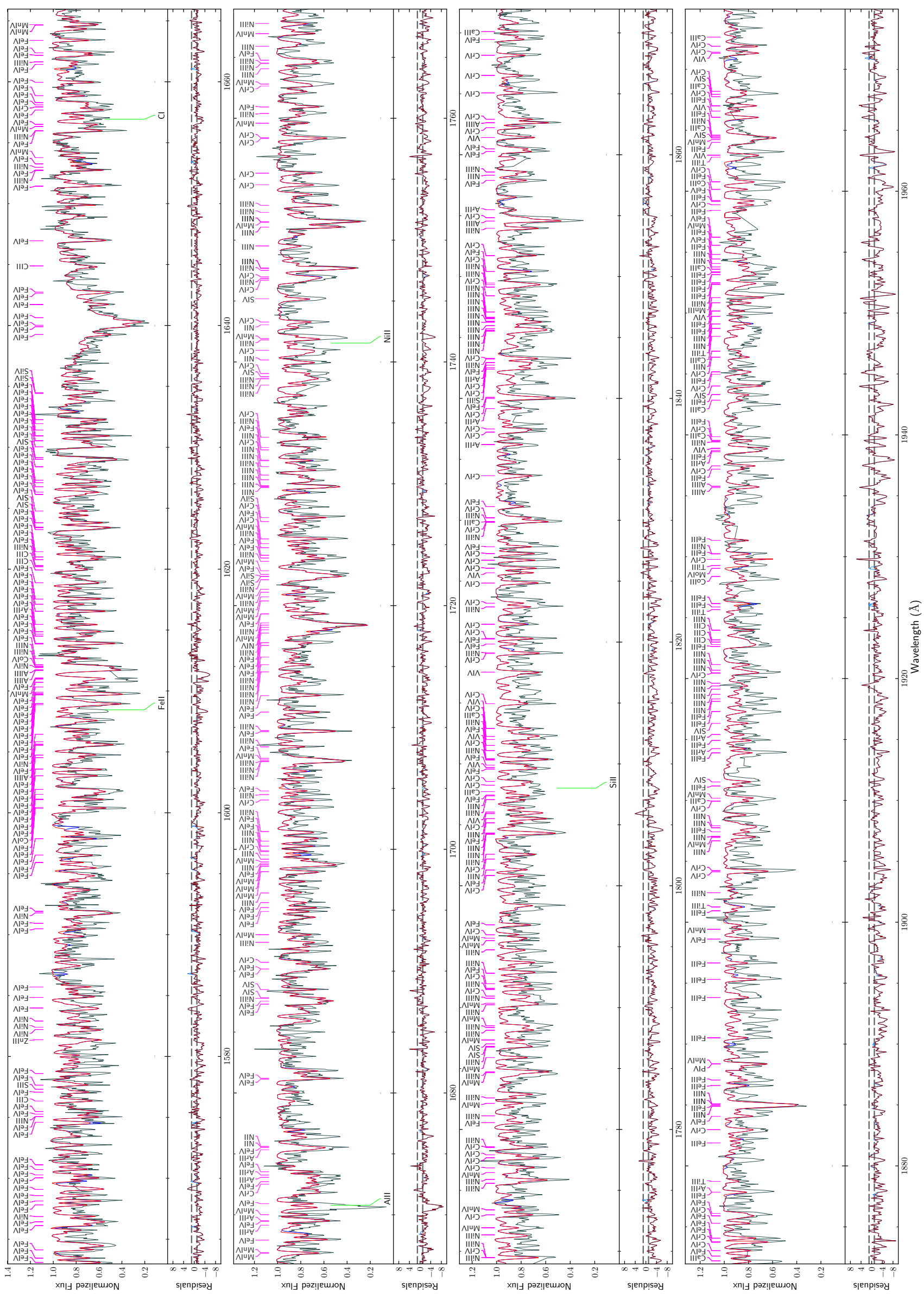

Fig. A.3. continued. 


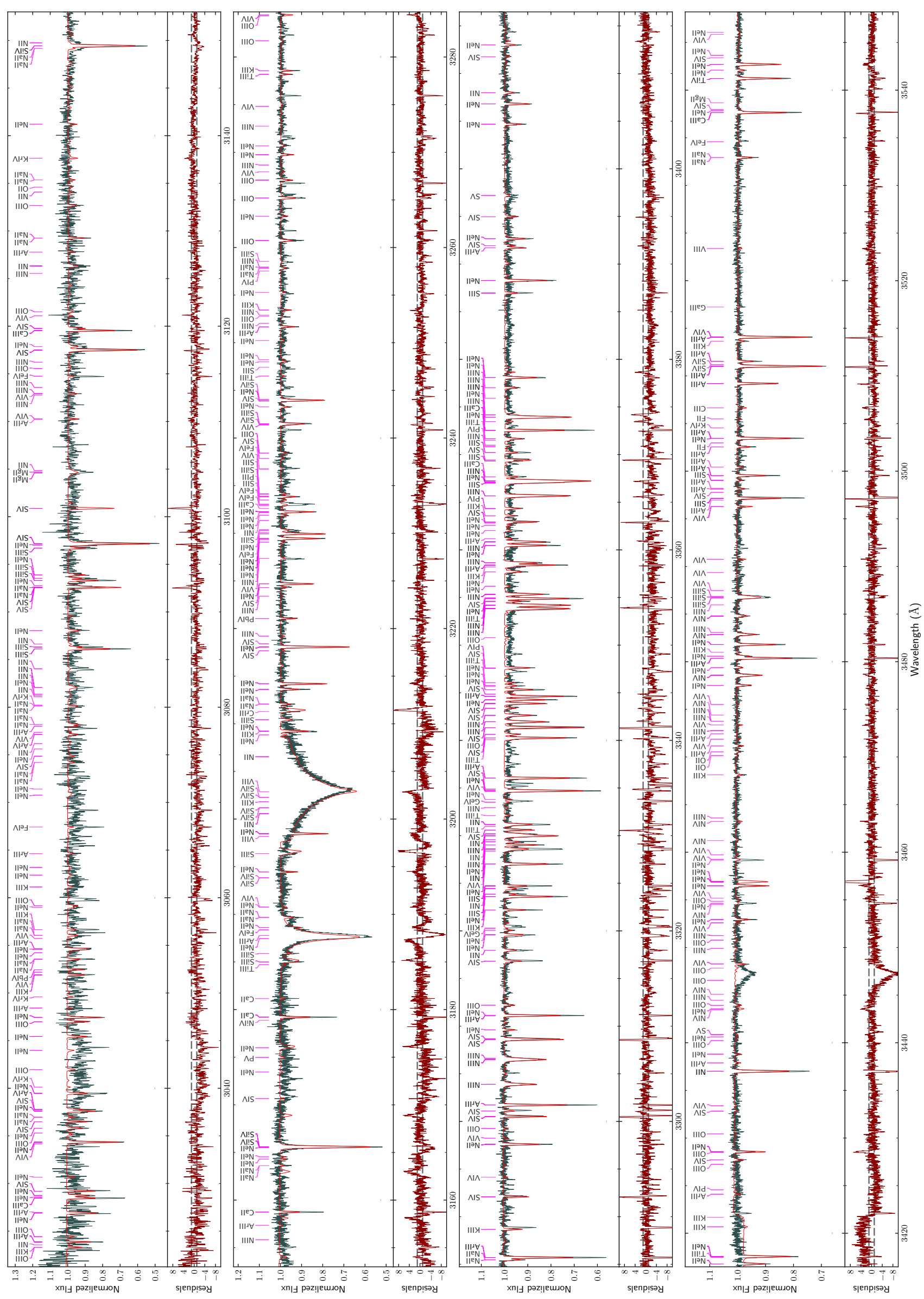

Fig. A.4. HIRES spectrum of HZ 44 (gray) and the final model (red). 


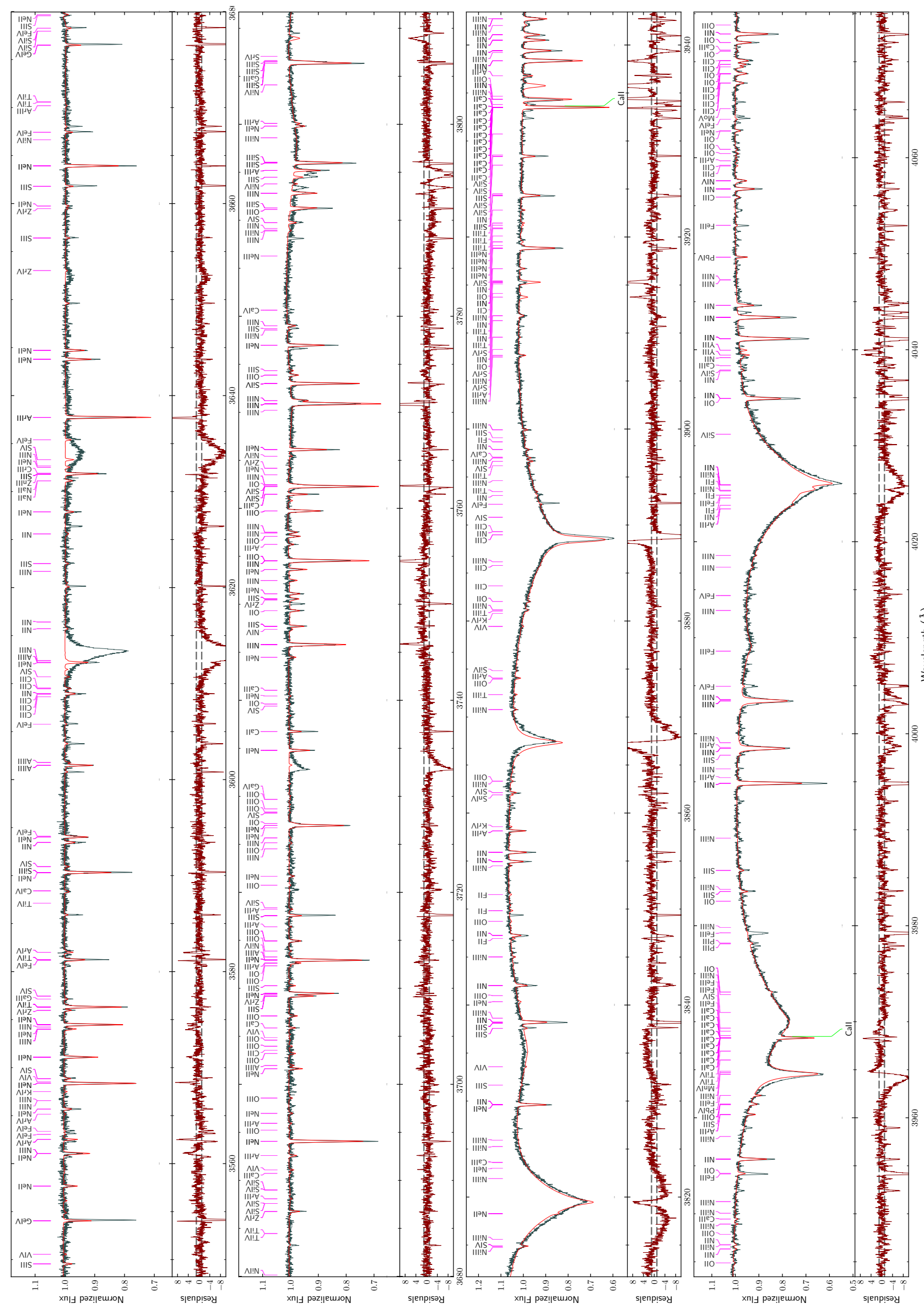

Fig. A.4. continued. 


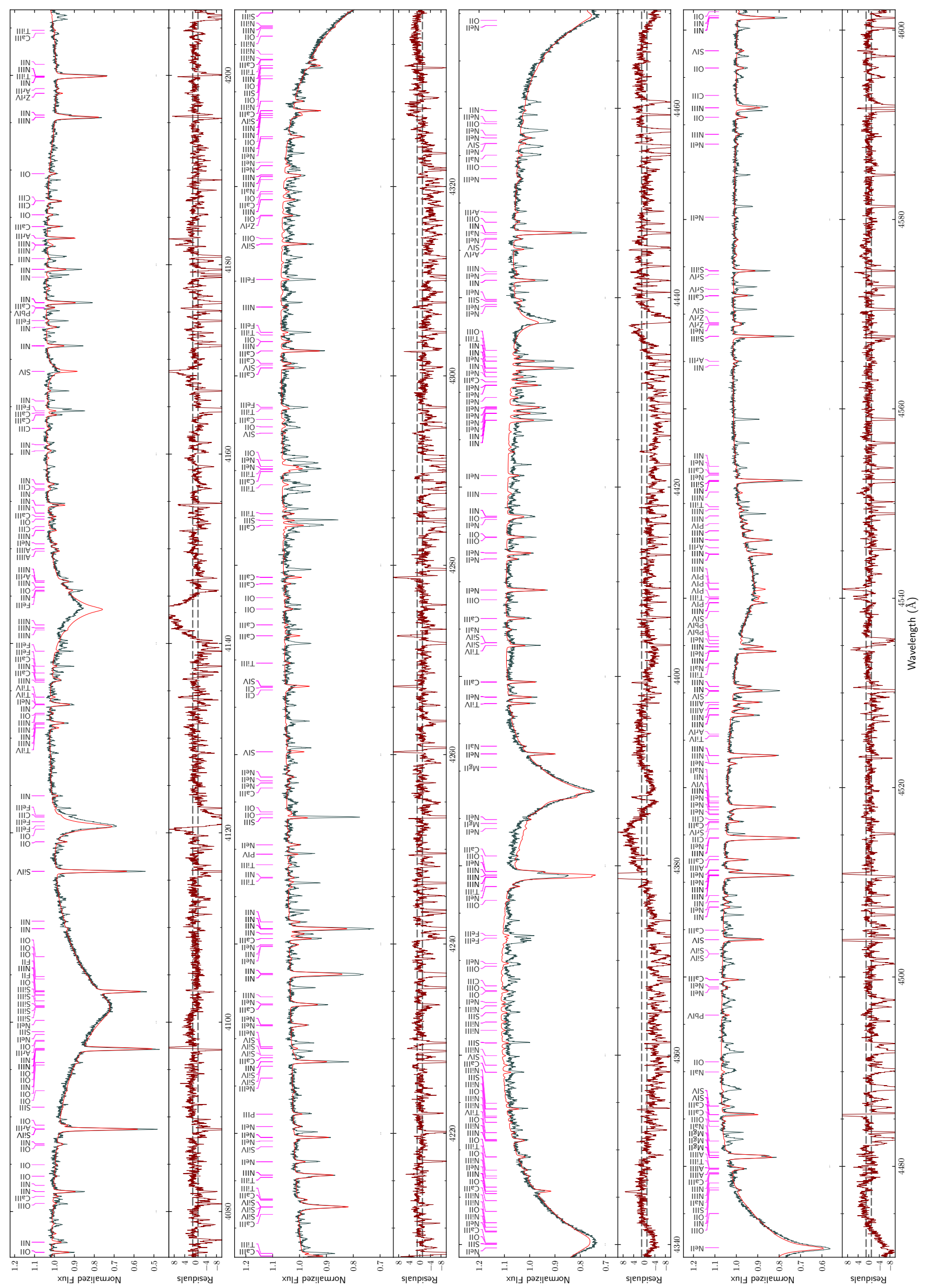

Fig. A.4. continued. 


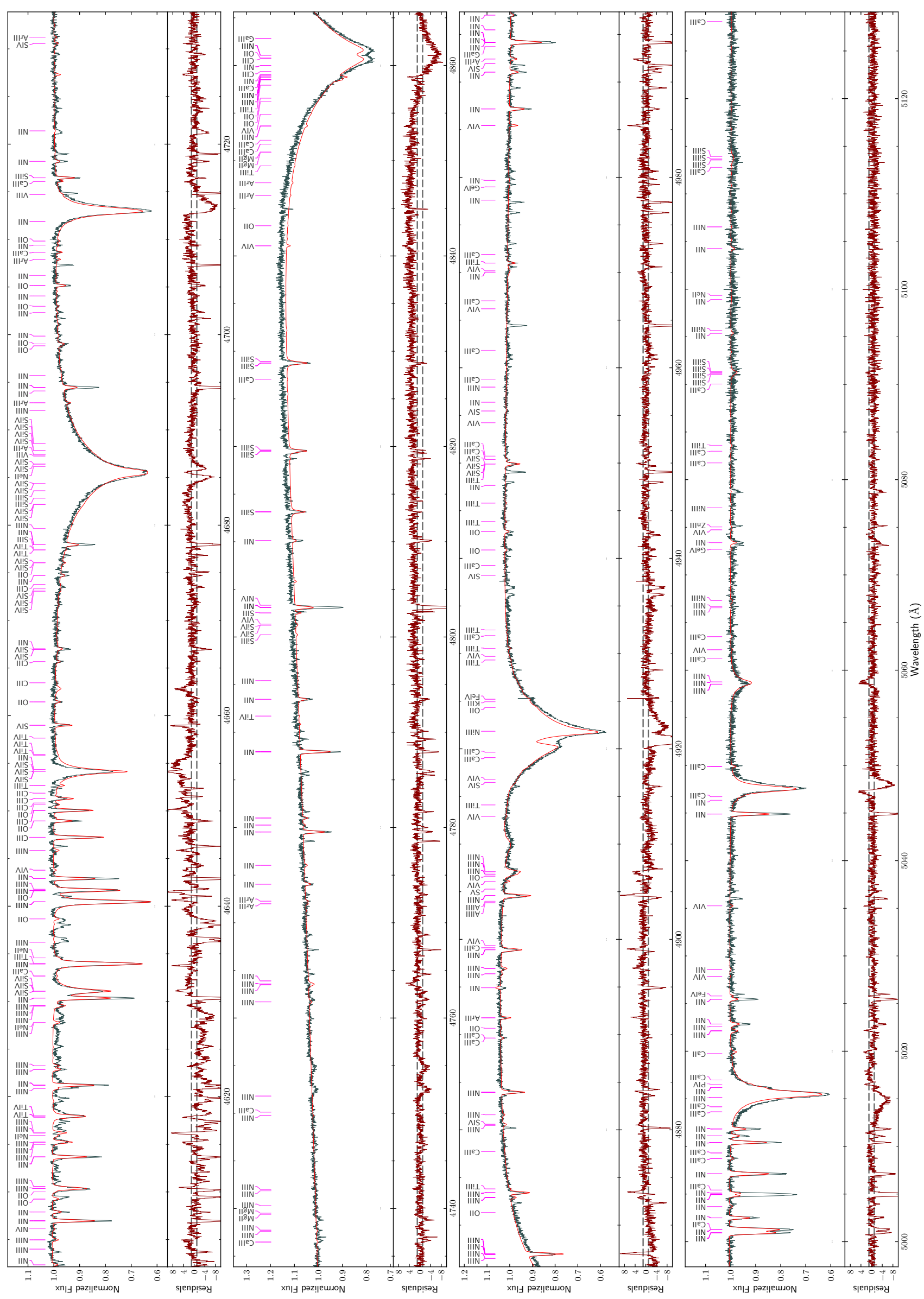

Fig. A.4. continued. 
A\&A 630, A130 (2019)

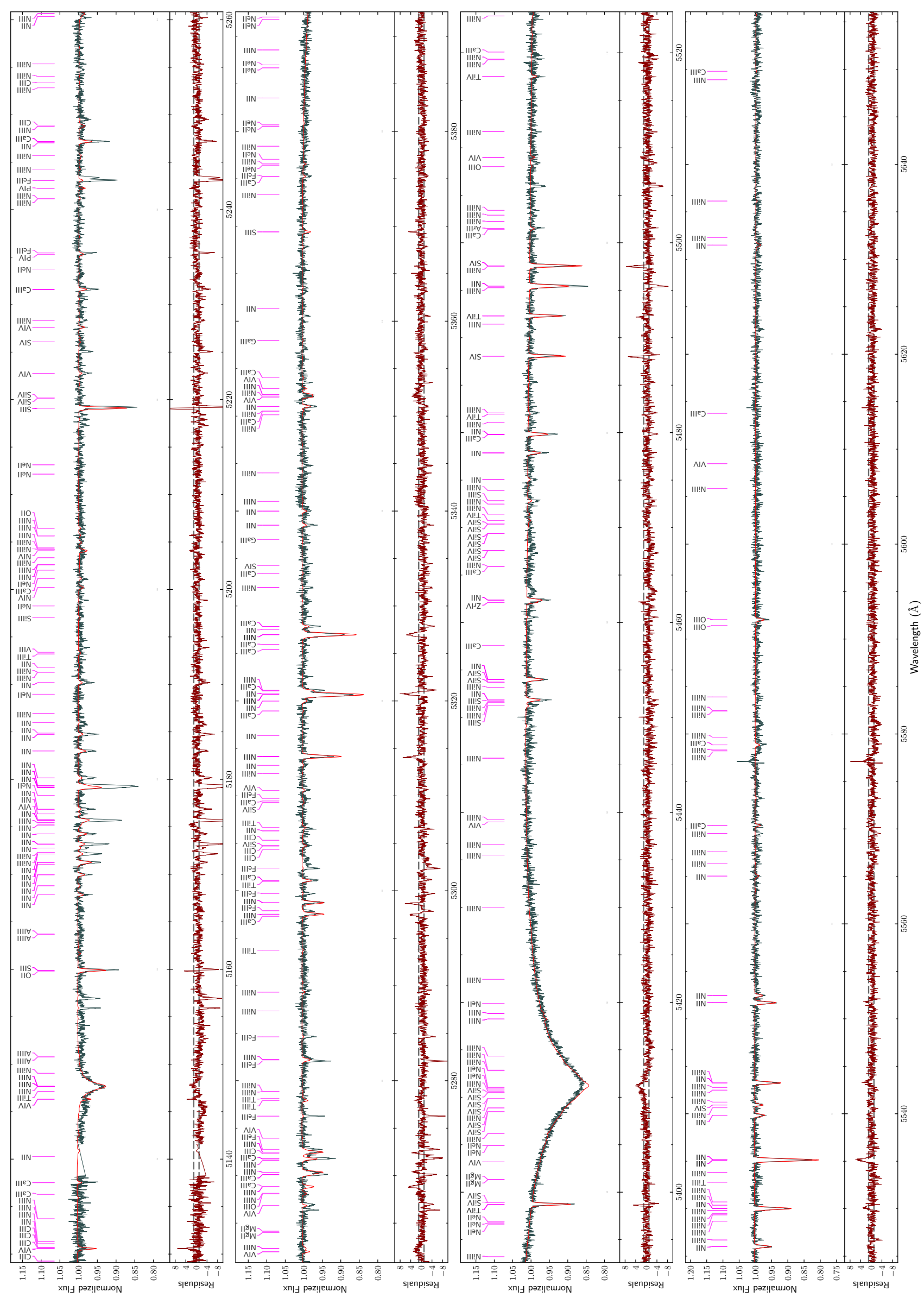

Fig. A.4. continued.

A130, page 34 of 46 


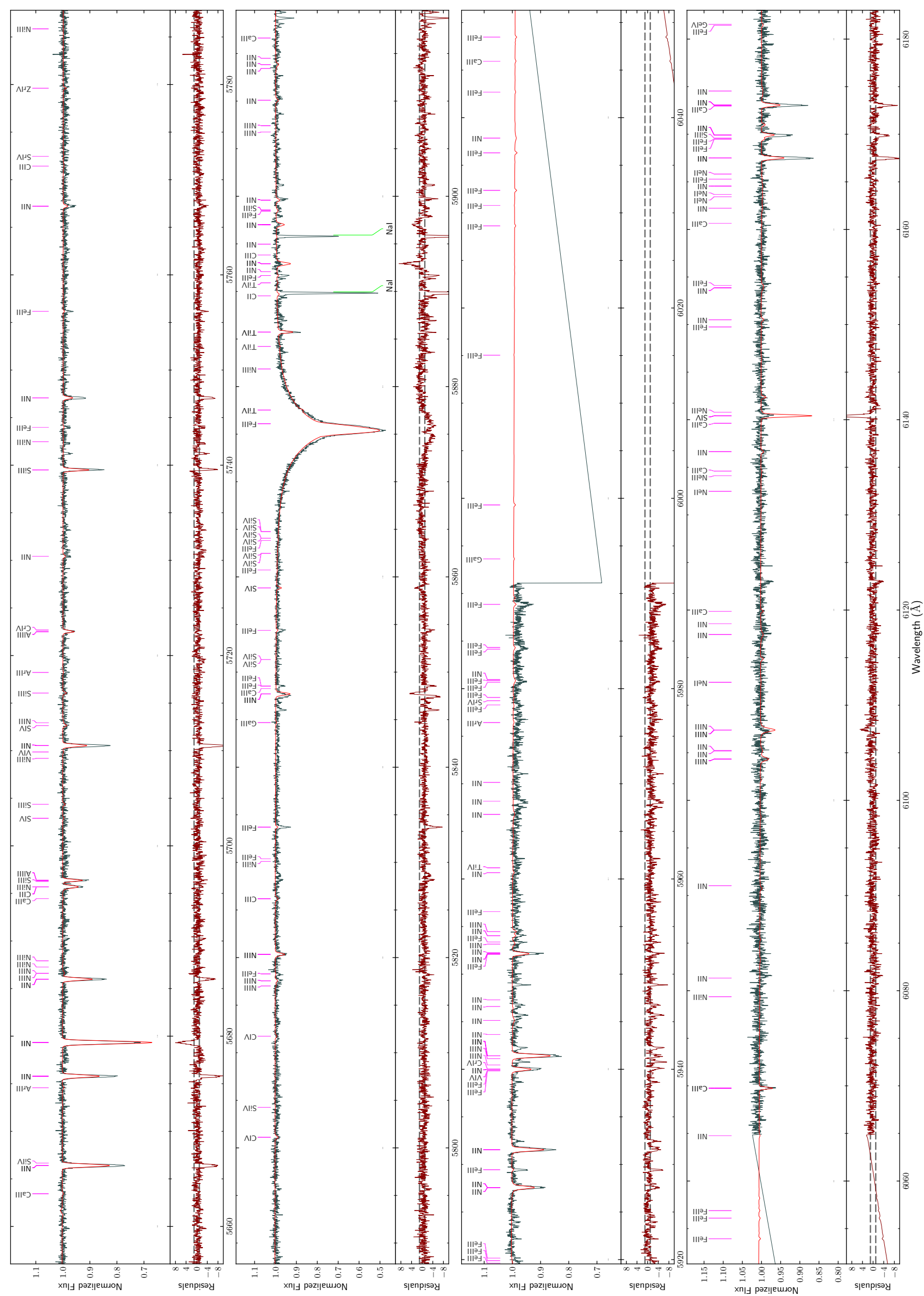

Fig. A.4. continued. 
A\&A 630, A130 (2019)

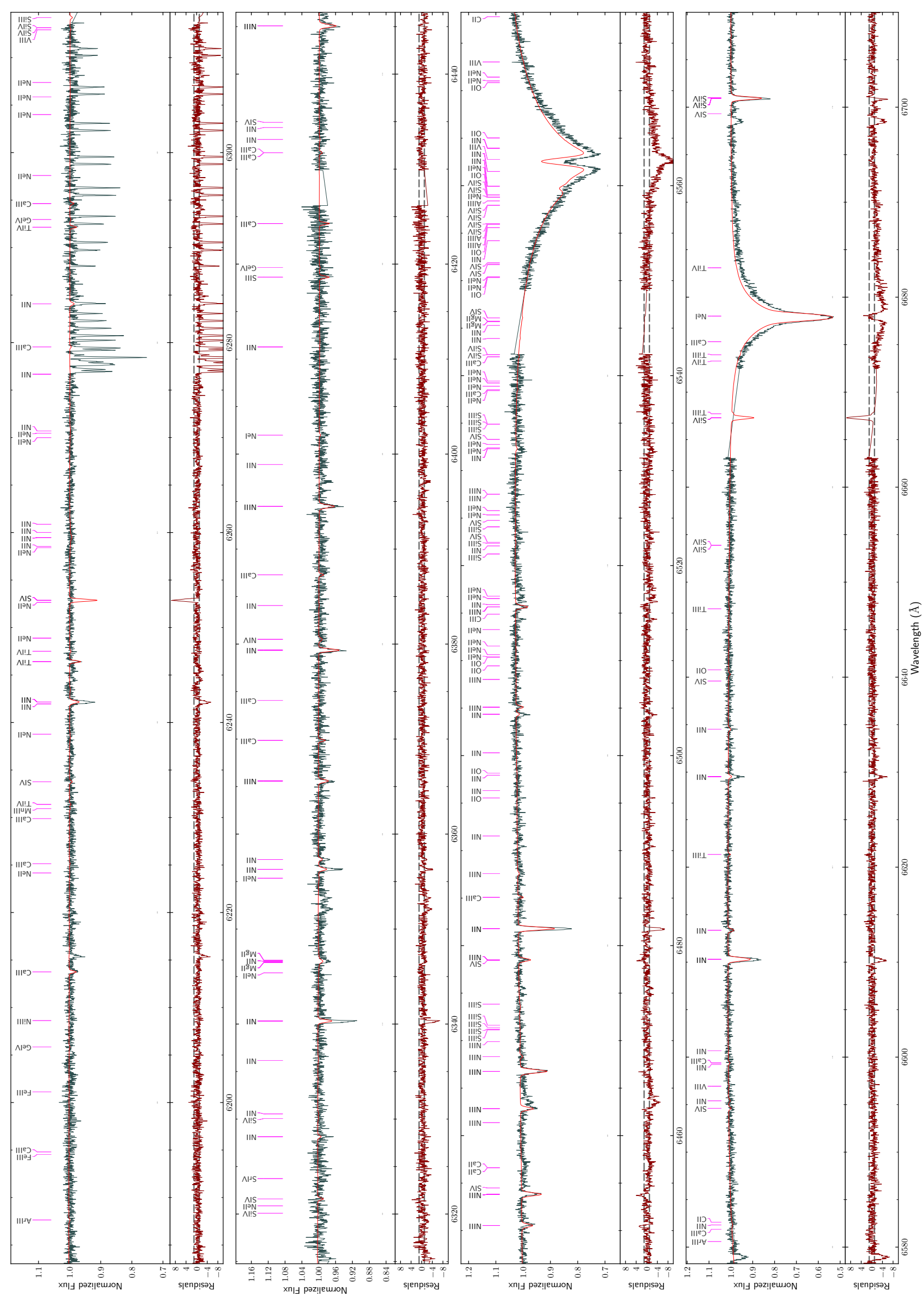

Fig. A.4. continued.

A130, page 36 of 46 


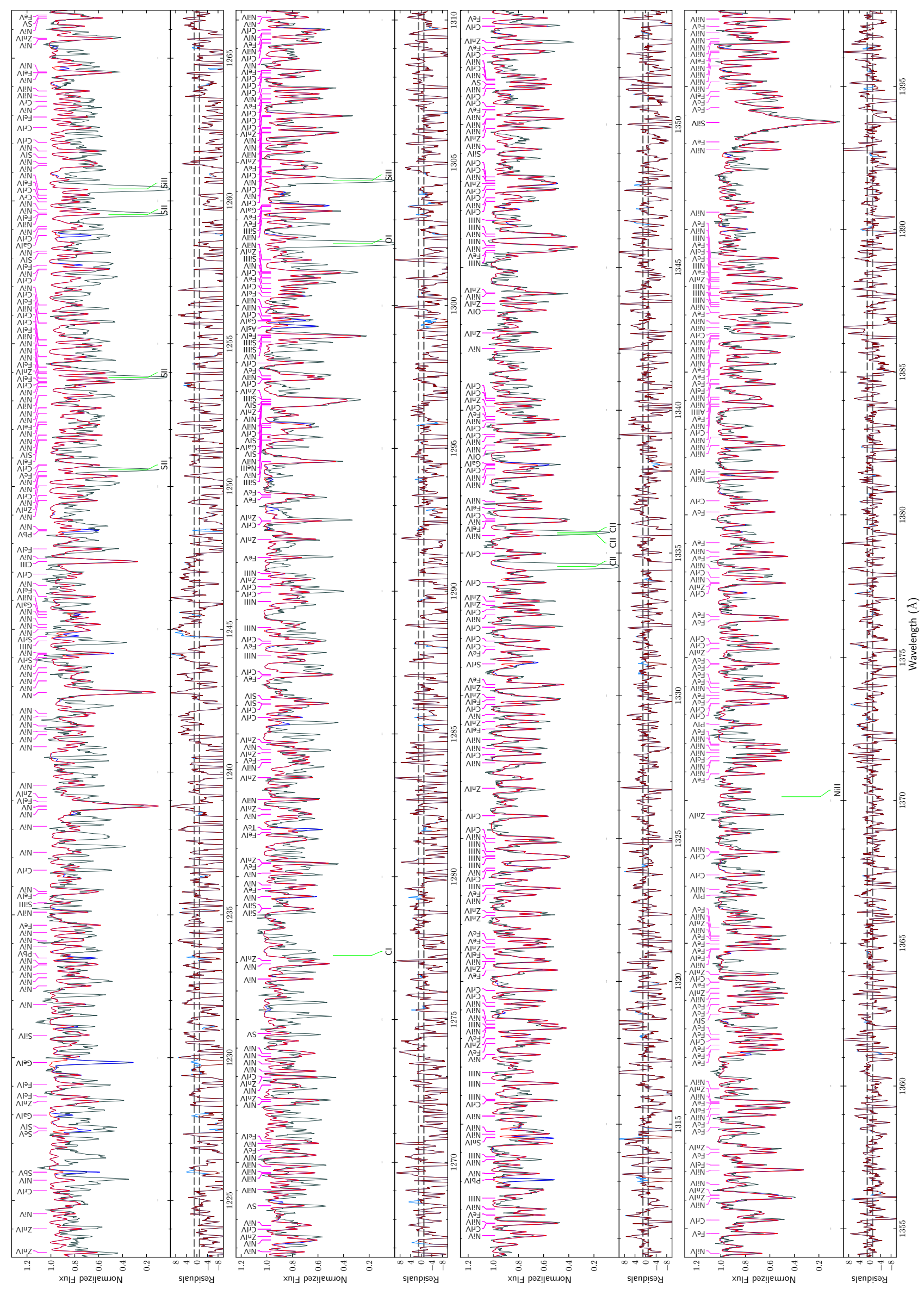

Fig. A.5. GHRS spectrum of HD 127493 (gray) and the final model (red, with heavy metals: blue). 


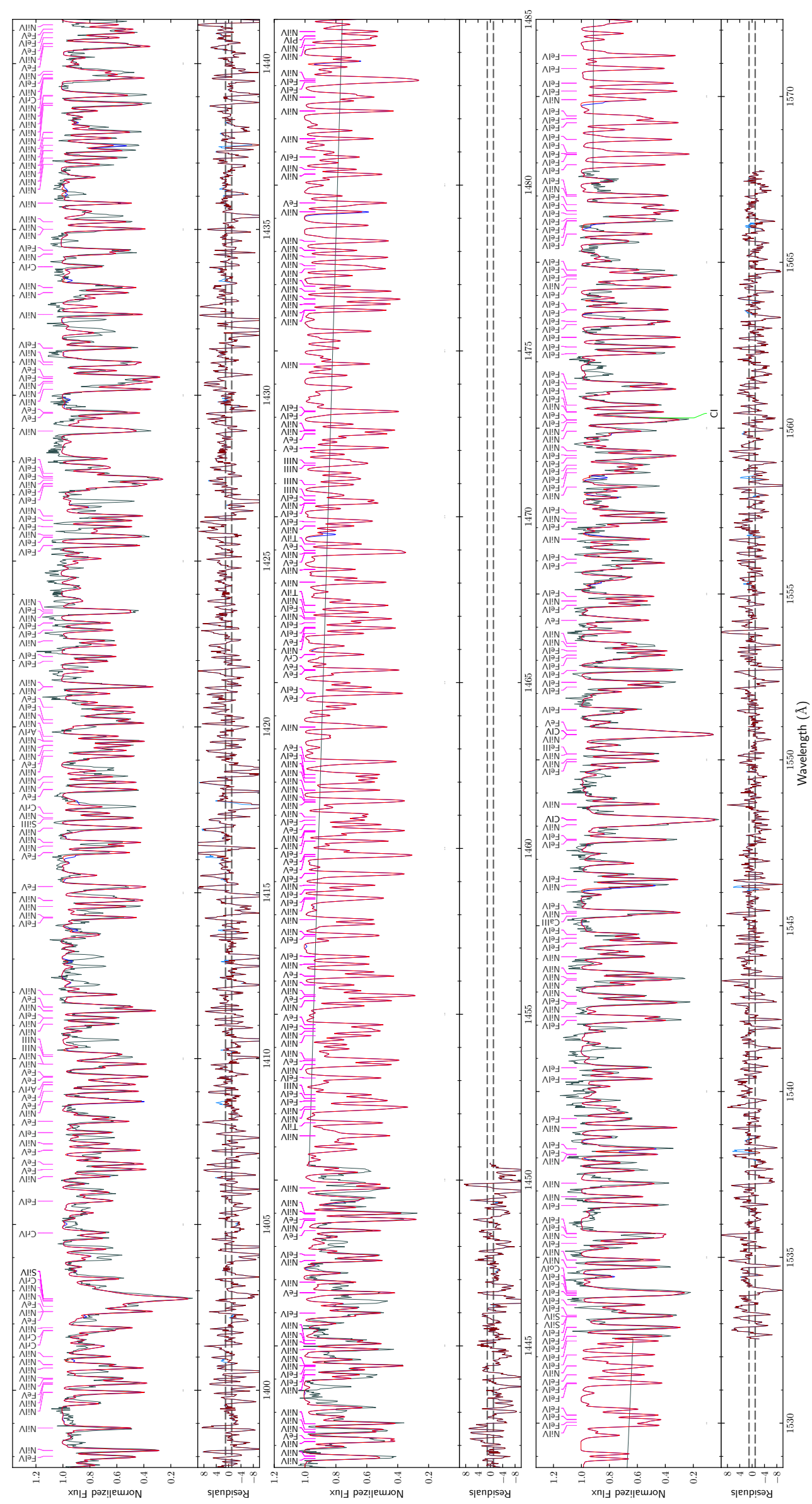

Fig. A.5. continued. 


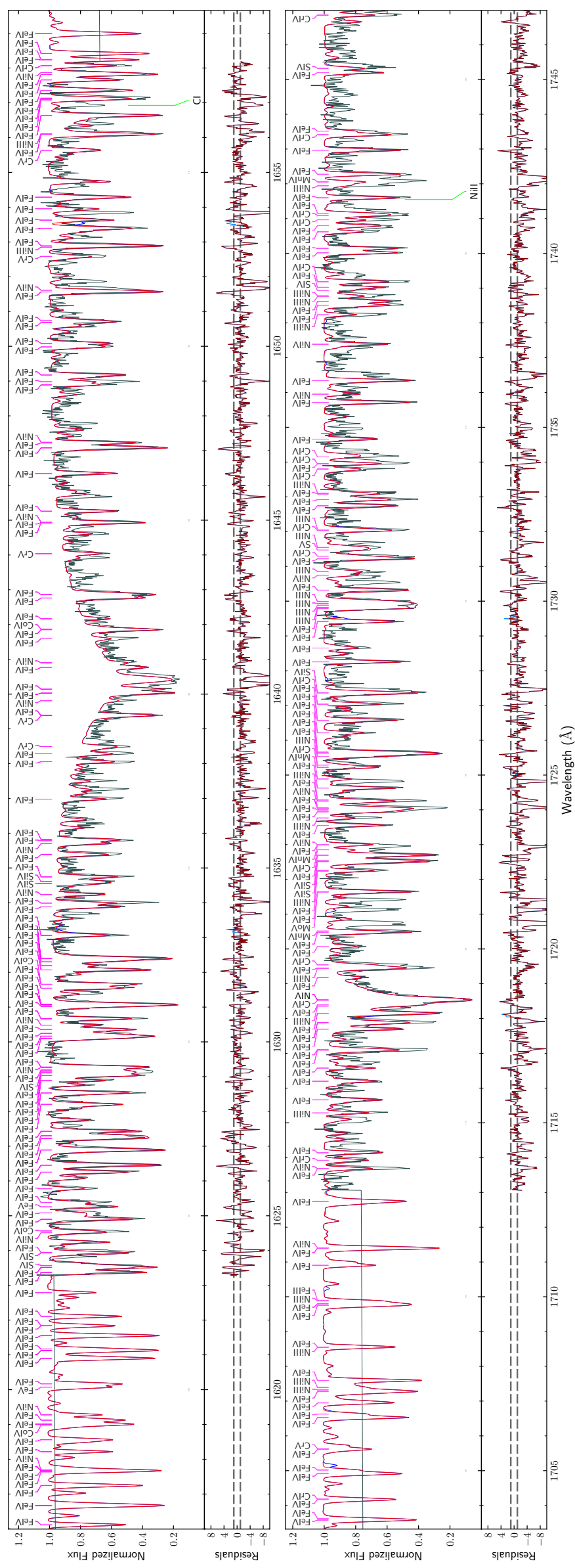

Fig. A.5. continued. 


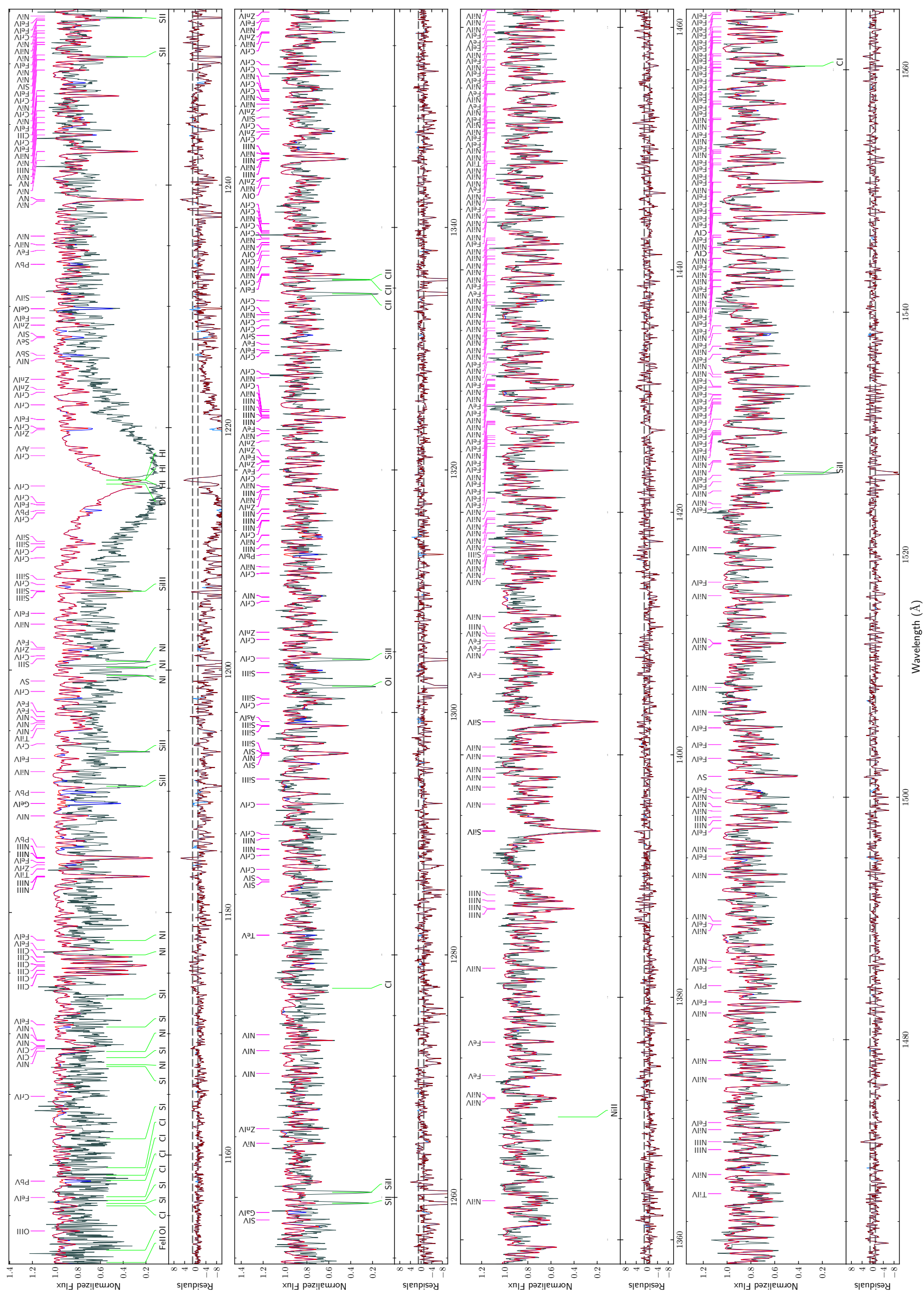

Fig. A.6. IUE spectrum of HD 127493 (gray) and the final model (red, with heavy metals: blue). 


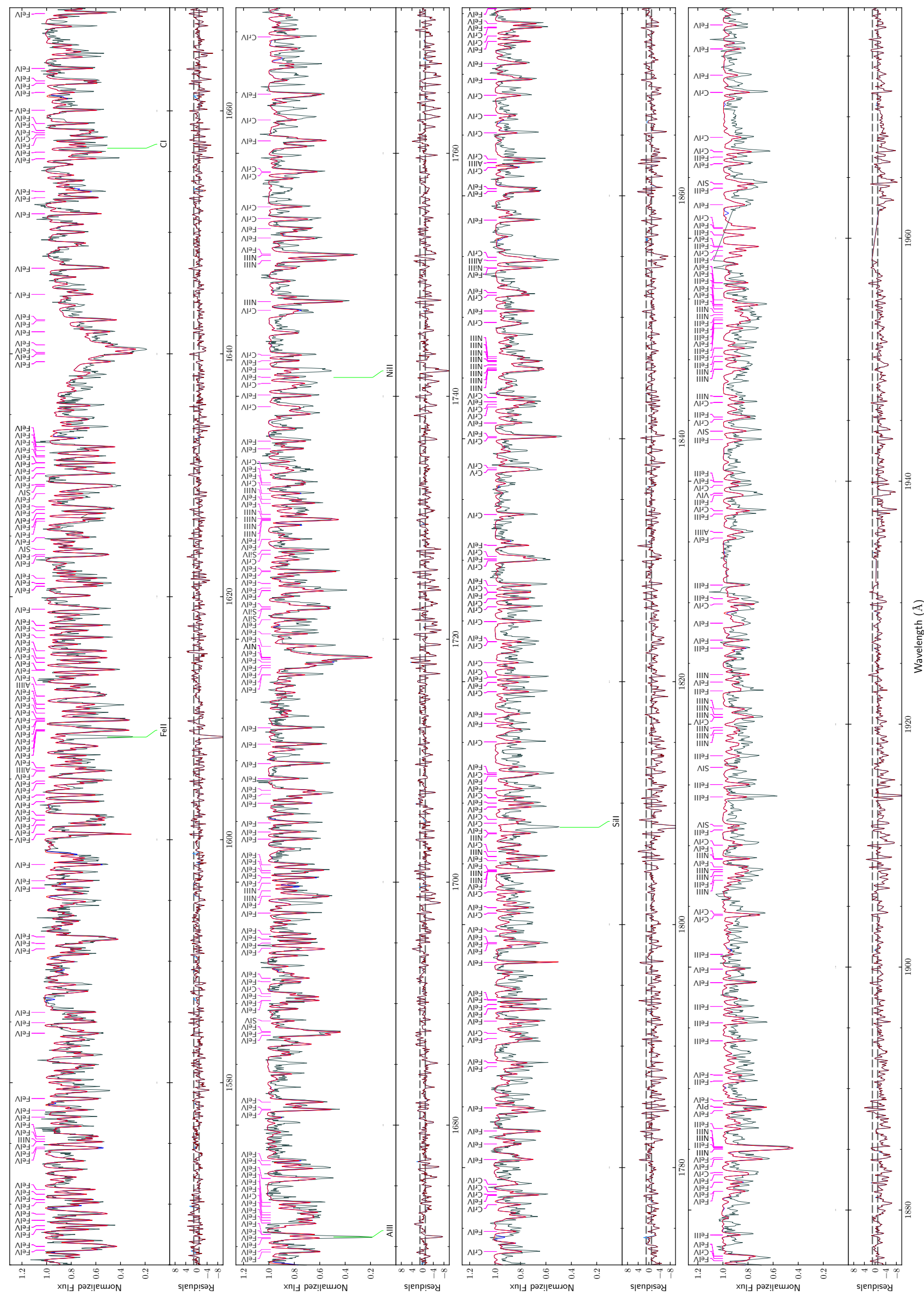

Fig. A.6. continued. 
A\&A 630, A130 (2019)

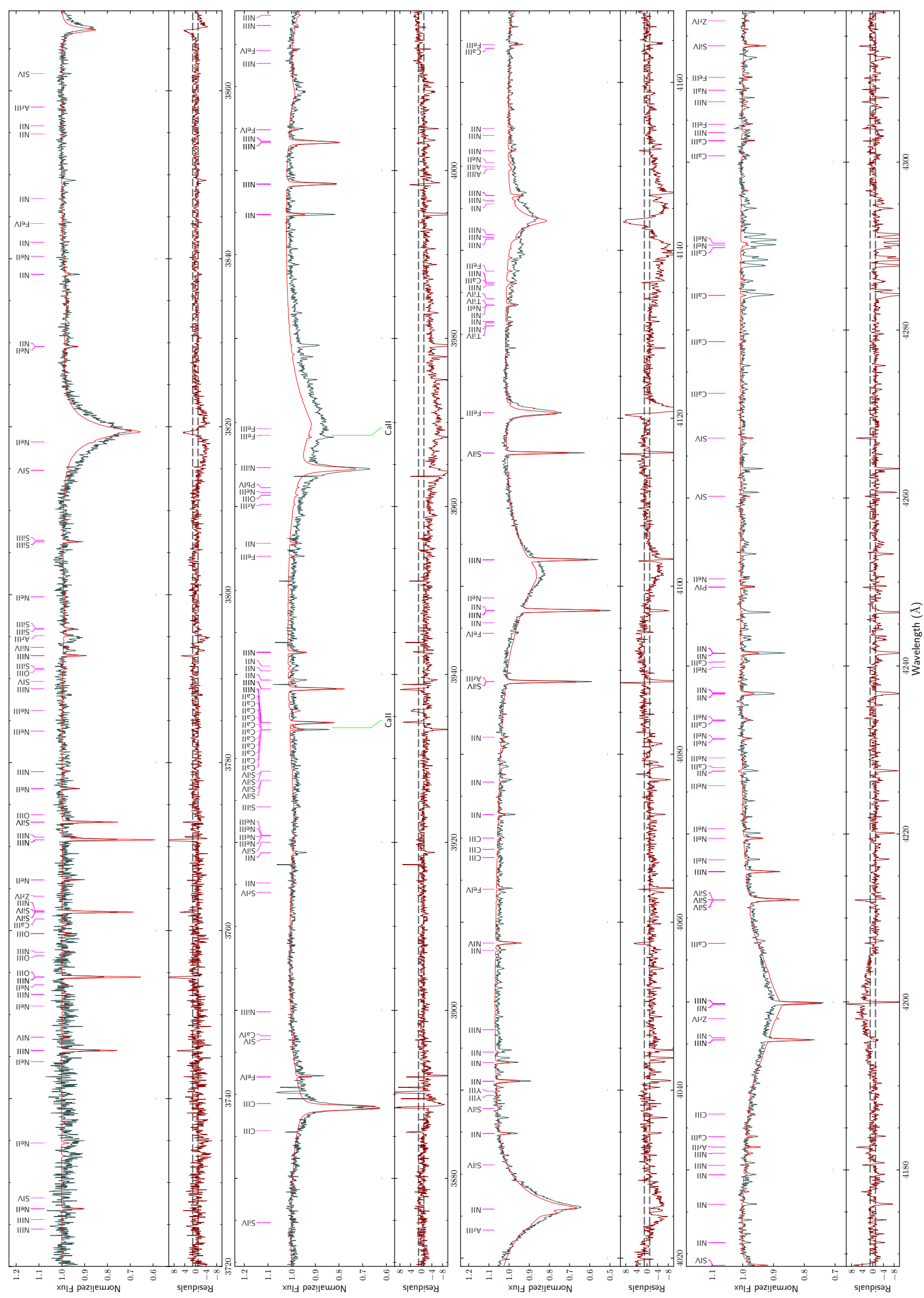

Fig. A.6. continued.

A130, page 42 of 46 


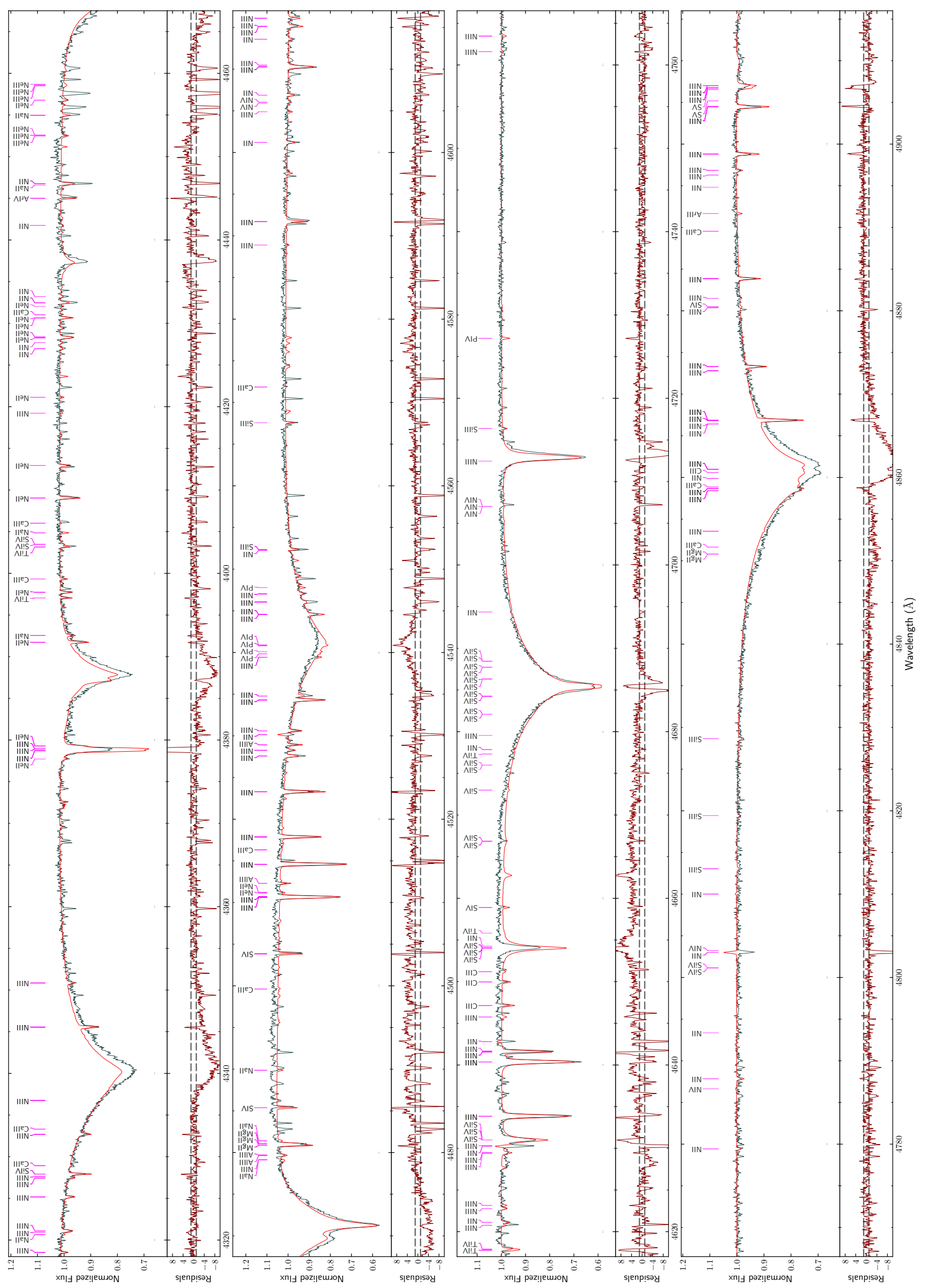

Fig. A.6. continued. 
A\&A 630, A130 (2019)

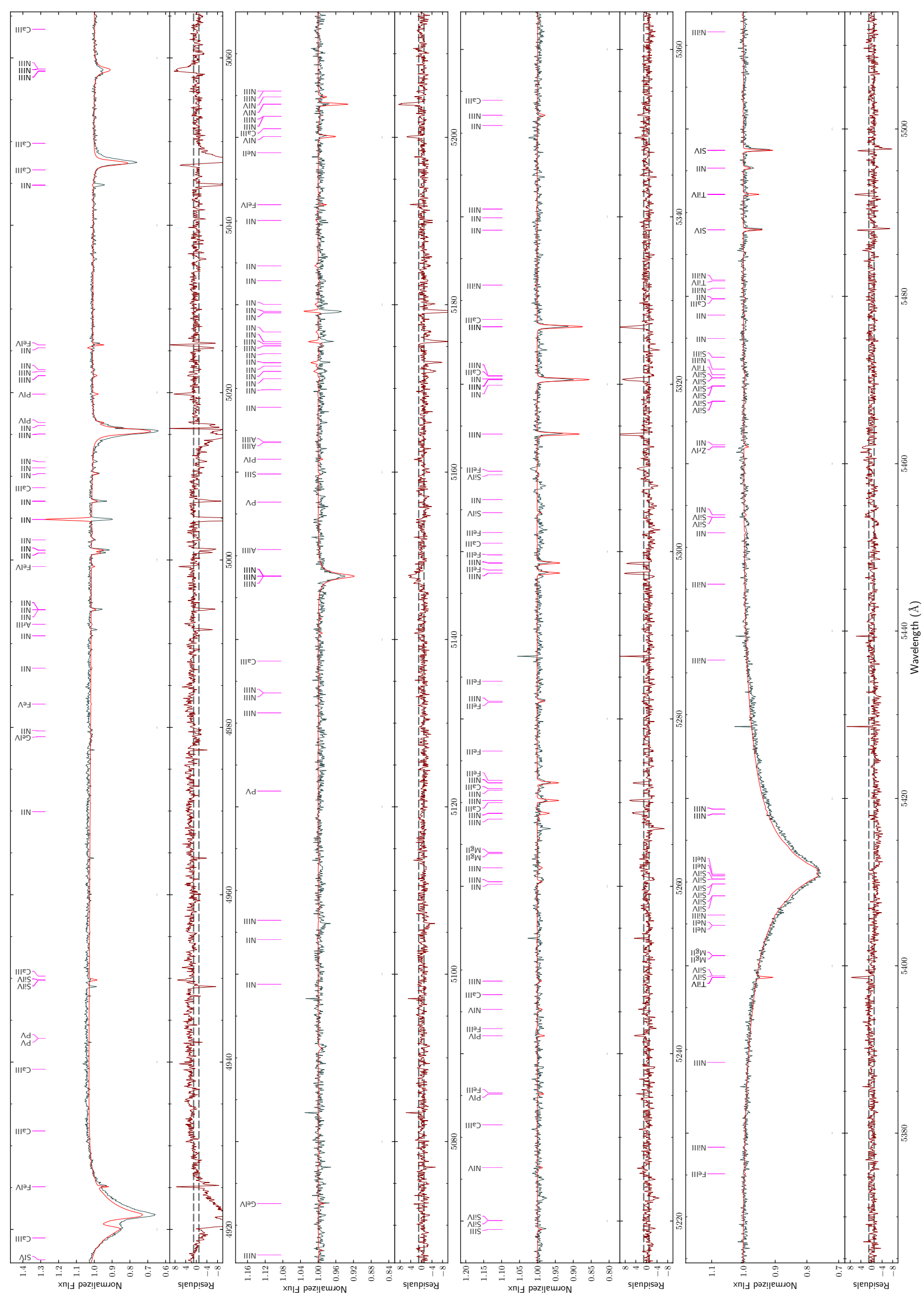

Fig. A.6. continued.

A130, page 44 of 46 


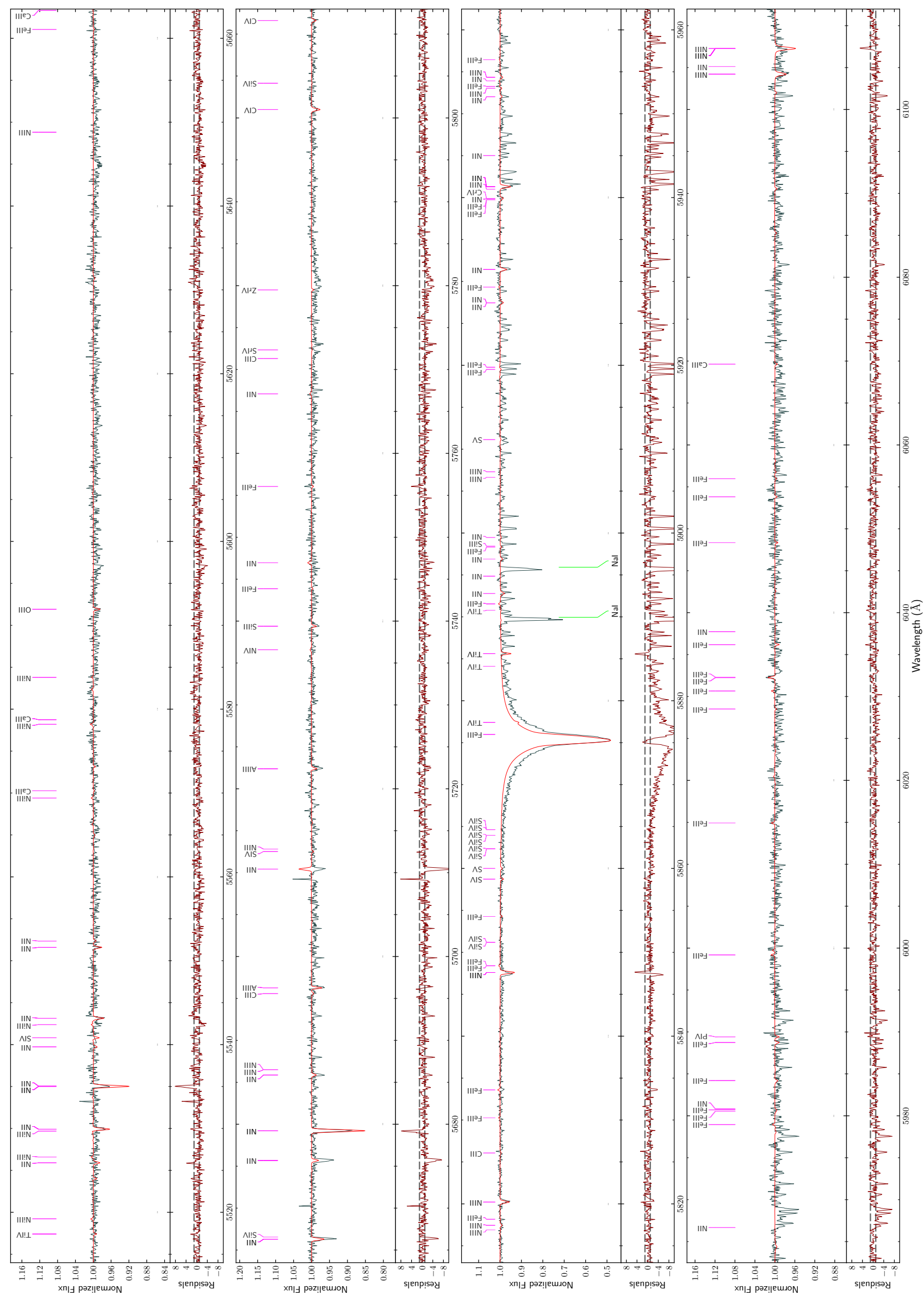

Fig. A.6. continued. 
A\&A 630, A130 (2019)

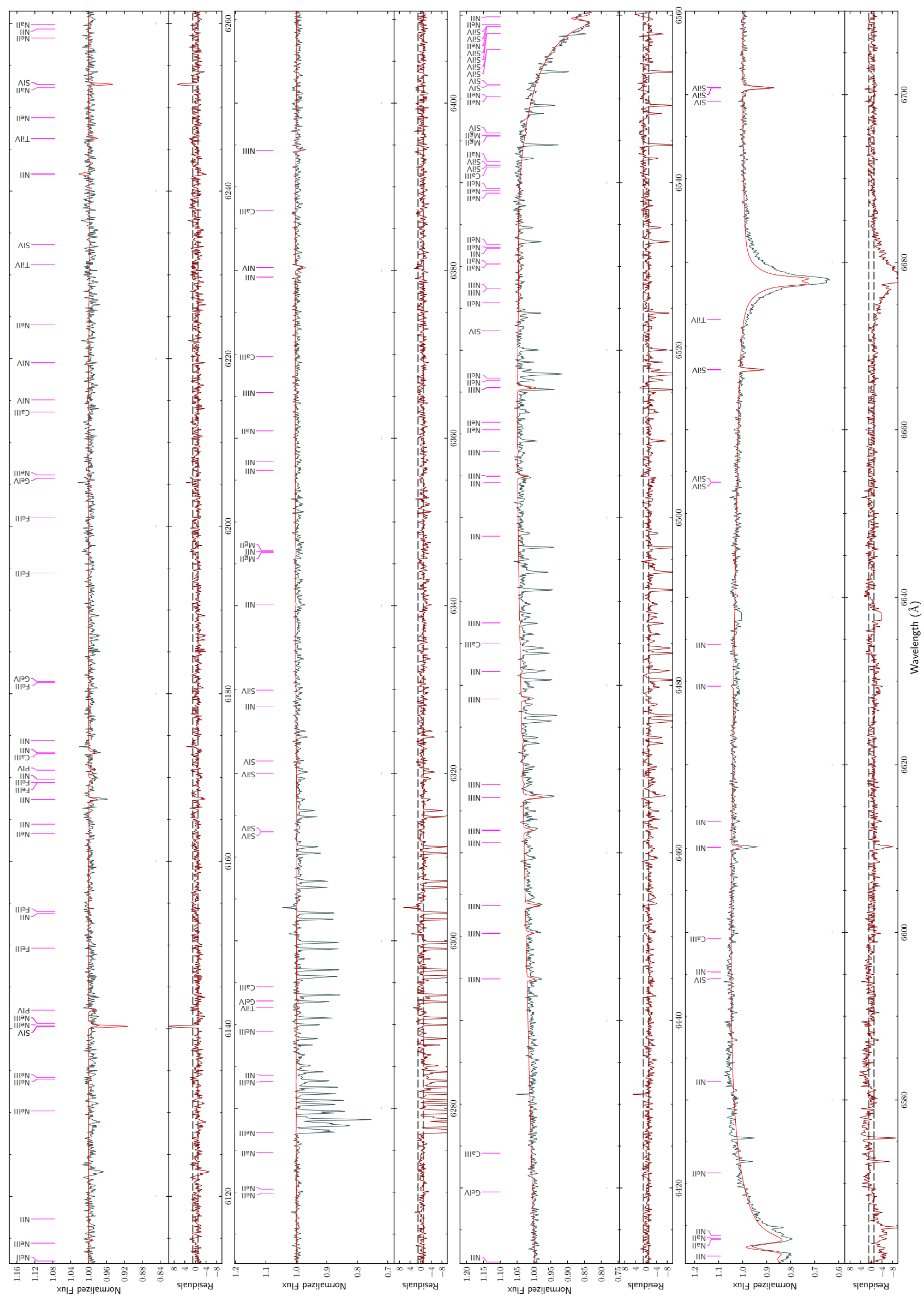

Fig. A.6. continued.

A130, page 46 of 46 